\title{
Real homotopy theory of semi-algebraic sets
}

\author{
ROBERT HARDT \\ PASCAL LAMBRECHTS \\ VICTOR TURCHIN \\ ISMAR VOLIĆ
}

\begin{abstract}
We complete the details of a theory outlined by Kontsevich and Soibelman that associates to a semi-algebraic set a certain graded commutative differential algebra of "semi-algebraic differential forms" in a functorial way. This algebra encodes the real homotopy type of the semi-algebraic set in the spirit of the de Rham algebra of differential forms on a smooth manifold. Its development is needed for Kontsevich's proof of the formality of the little cubes operad.
\end{abstract}

14P10, 55P62

\section{Introduction}

We start with a brief review of rational homotopy theory whose beginnings go back to Sullivan [24] and whose full development can be found in Bousfield and Gugenheim [2] and Félix, Halperin and Thomas [7]. To any topological space $X$, one can associate in a functorial way a certain commutative differential graded algebra (or CDGA for short) $\operatorname{APL}_{P L}(X)$ with coefficients in $\mathbb{Q}$. The main feature of this algebra is that if $X$ is nilpotent (eg, simply connected) with finite Betti numbers, then any CDGA $(A, d)$, where $d$ is the differential, that is quasi-isomorphic to $\mathrm{A}_{\mathrm{PL}}(X)$ contains all the information about the rational homotopy type of $X$. For example, an analog of the de Rham theorem implies that the rational singular cohomology algebra of $X$ is given by the homology of $(A, d)$, ie $\mathrm{H}^{*}(X ; \mathbb{Q}) \cong \mathrm{H}(A, d)$. Another procedure also recovers $\pi_{*}(X) \otimes \mathbb{Q}$, the rational homotopy groups of $X$, from $(A, d)$ [24].

Sullivan's construction was inspired by de Rham theory which associates to a smooth manifold $M$ a commutative differential graded algebra of smooth differential forms $\Omega_{\mathcal{C}^{\infty}}^{*}(M)$ with coefficients in $\mathbb{R}$. In fact, the de Rham and Sullivan constructions are related because $\Omega_{\mathcal{C}^{\infty}}^{*}(M)$ is quasi-isomorphic to $\mathrm{APL}_{\mathrm{PL}}(M ; \mathbb{R}):=\mathrm{A}_{\mathrm{PL}}(M) \otimes \mathbb{Q} \mathbb{R}$. One loses some information about the rational homotopy type of $M$ after tensoring with $\mathbb{R}$ but what is left (the "real homotopy type") is still valuable. For example, one can still recover the real singular cohomology (this is the classical de Rham's theorem) or the 
dimension of the rational homotopy groups. In some applications, the de Rham CDGA of smooth forms is even more manageable than $A_{P L}(M ; \mathbb{R})$ because it is closer to the geometry of the manifold. For instance, a variation of this approach is used to prove that compact Kähler manifolds are formal in Deligne, Griffiths, Morgan and Sullivan [4].

The goal of the present paper is to supply the details of a theory sketched by Kontsevich and Soibelman in [19, Appendix 8] which produces an analog of the de Rham differential algebra for semi-algebraic sets (subsets of $\mathbb{R}^{n}$ defined by finitely many polynomial equations, inequalities and boolean operations). More precisely, following their approach, we will construct a functor

$$
\begin{aligned}
\Omega_{\mathrm{PA}}^{*}: \text { SemiAlg } & \longrightarrow \text { CDGA } \\
X & \longmapsto \Omega_{\mathrm{PA}}^{*}(X)
\end{aligned}
$$

where SemiAlg is the category of semi-algebraic sets and $\Omega_{\mathrm{PA}}^{*}(X)$ is the CDGA with real coefficients of so-called PA forms on the semi-algebraic set $X$. Here "PA" stands for "piecewise semi-algebraic" which is the terminology coined by Kontsevich and Soibelman for describing a wider class of spaces than we will consider in this paper; nevertheless, we will retain the "PA" notation for the benefit of ease of translation between this paper and the Kontsevich-Soibelman one. Our main result is Theorem 6.1 which we restate here.

Theorem 1.1 For $X$ a compact semi-algebraic set, $\Omega_{\mathrm{PA}}^{*}(X)$ and $\mathrm{A}_{\mathrm{PL}}(X ; \mathbb{R})$ are connected by a zigzag of natural transformations of CDGAs that induce isomorphisms on homology.

This in particular implies that any CDGA quasi-isomorphic to $\Omega_{\mathrm{PA}}^{*}(X)$ contains all the information about the real homotopy type of a compact semi-algebraic set $X$.

Our main motivation for supplying the details of the proof of this theorem and various results related to it is that it is an essential ingredient in Kontsevich's proof of the formality of the little cubes operad in [18, Section 3]. Details of this formality proof will be given by the second and fourth authors in [20].

\subsection{Outline of the paper}

We build on [19, Appendix 8], which gives a good first idea of the theory. The roadmap of the present paper is as follows.

In Section 2, we review semi-algebraic sets and their stratifications; some PL topology and the semi-algebraic Hauptvermutung; and the notion of a current which is a convenient alternative to a singular chain in $\mathbb{R}^{n}$. 
In Section 3, we define semi-algebraic chains in terms of currents (this corresponds to what Kontsevich and Soibelman call chains in [19]). Briefly, a $k$-chain $\gamma \in \mathrm{C}_{k}(X)$ in a semi-algebraic set $X \subset \mathbb{R}^{n}$ is represented by a linear combination of bounded semi-algebraic oriented smooth $k$-dimensional submanifolds $V_{i}$ in $\mathbb{R}^{n}$ whose closure is contained in $X$. By a remarkable property of semi-algebraic sets, the $k$-volume of the $V_{i}$ is finite. Any two such linear combinations $\sum n_{i} \llbracket V_{i} \rrbracket$ and $\sum n_{j}^{\prime} \llbracket V_{j}^{\prime} \rrbracket$ are declared to be equal if, for any smooth form with compact support in $\mathbb{R}^{n}$,

$$
\sum n_{i} \int_{V_{i}} \omega=\sum n_{j}^{\prime} \int_{V_{j}^{\prime}} \omega .
$$

In other words, two chains are equal if they are equal as currents. This defines a chain complex $\mathrm{C}_{*}(X)$ whose boundary $\partial$ is the classical boundary of currents. In order to prove that $\partial\left(\mathrm{C}_{k}(X)\right) \subset \mathrm{C}_{k-1}(X)$ and that a semi-algebraic map $f: X \rightarrow Y$ induces a chain map, we need to establish a generalization of Stokes' Theorem to semi-algebraic manifolds, which is Theorem 3.5. In this section, we also construct a natural cross product $\times: \mathrm{C}_{*}(X) \otimes \mathrm{C}_{*}(Y) \rightarrow \mathrm{C}_{*}(X \times Y)$.

In Section 4, we develop a suitable notion of convergence for sequences of chains. This will be important for showing that some cochains are determined by their value on a dense subset, ie at generic points.

In Section 5, we build the cochain complex of PA forms. We begin this process in Section 5.1 by defining the cochain complex $C^{*}(X)$ with coefficients in $\mathbb{R}$ as the dual of $\mathrm{C}_{*}(X)$. The CDGA of PA forms that we are after will be a subcomplex $\Omega_{\mathrm{PA}}^{*}(X) \subset \mathrm{C}^{*}(X)$. Along the way, we first define a subcomplex of minimal forms $\Omega_{\text {min }}^{*}(X) \subset \mathrm{C}^{*}(X)$ in Section 5.2 and show that it is equipped with a graded commutative cross product. The key property necessary for the proof of the existence of that product is the fact that a minimal form is smooth at a generic point, which is the content of Proposition 5.7.

The problem with $\Omega_{\min }^{*}(X)$ is that it does not satisfy the de Rham isomorphism. We thus need to extend this algebra in order to have the right homotopical properties. For this, we first define in Section 5.3 a notion of strongly continuous family of chains which allows us to construct the desired cochain complex of PA forms $\Omega_{\mathrm{PA}}^{*}(X)$ in Section 5.4, where we also exhibit an algebra structure on $\Omega_{\mathrm{PA}}^{*}(X)$ (the proof turns out to be more involved than for minimal forms).

In Section 6, we prove Theorem 1.1. The proof mainly follows the scheme of the analog result for the de Rham algebra of smooth manifolds. The main difference is that our proof of the Poincare Lemma is more complicated and it relies on the semi-algebraic Hauptvermutung. 
In Section 7, we show that the weak equivalence established in the previous section are monoidal, and we prove other monoidal equivalences. This is useful in applications to operads, which is our main motivation.

In Section 8, we study integration along the fiber for semi-algebraic bundles whose fibers are compact oriented manifolds.

In Section 9, we discuss some differences between this paper and [19, Appendix 8] and explain why we were unable to prove some of the claims made there.

Acknowledgements We thank Thierry De Pauw for many hours of discussion about currents, for his invaluable help in the proof of Theorem 3.5, and for finding many results we needed in Federer [6]. We thank Yves Félix, Don Stanley, Maxim Kontsevich, and Yan Soibelman for fruitful conversations. We warmly thank Greg Arone for his patience and encouragements to finish this paper. We also thank Michel Coste for help with semi-algebraic sets and Lou van den Dries for answering questions and pointing out the reference by Comte, Lion and Rolin [3].

The first, third and fourth authors were supported in part by the National Science Foundation grants DMS-0604605, DMS-0504390 and DMS-0968046. The second author is Maître de Recherche au FNRS.

\section{Background}

\subsection{Review of semi-algebraic sets and stratifications}

A short summary of semi-algebraic sets with definitions and most of the properties that we will need can be found in Hardt [12, Section 1] and a complete reference is Bochnak, Coste and Roy [1]. Briefly, a subset $X$ of $\mathbb{R}^{m}$ is semi-algebraic if it is a finite union of finite intersections of solution sets of polynomial equations and inequalities. A semi-algebraic map is a continuous map of semi-algebraic sets $X \subset \mathbb{R}^{m}$ and $Y \subset \mathbb{R}^{n}$ whose graph is a semi-algebraic set in $\mathbb{R}^{m} \times \mathbb{R}^{n}$. By a semi-algebraic function we will mean a semi-algebraic map with values in $\mathbb{R}$. Note that in this paper we always assume that semi-algebraic maps and functions are continuous. Semi-algebraic sets are clearly stable under taking finite intersections, finite unions, and complements. Also, given a semi-algebraic set $X$, its closure $\bar{X}$ and its interior $\operatorname{int}(X)$ are semi-algebraic. The same is true for the image $f(X)$ or the preimage $f^{-1}(X)$ under a semi-algebraic map $f$ [1, page 23].

Definition 2.1 A semi-algebraic manifold of dimension $k$ is a semi-algebraic set such that each point has a semi-algebraic neighborhood semi-algebraically homeomorphic to $\mathbb{R}^{k}$ or to $\mathbb{R}_{+} \times \mathbb{R}^{k-1}$. 
Clearly a semi-algebraic manifold is a topological manifold with boundary, that is, a semi-algebraic manifold of dimension $k-1$. We can therefore talk about the orientability and the orientation of a semi-algebraic manifold as well as the induced orientation on the boundary.

We will also consider smooth submanifolds of $\mathbb{R}^{n}$, always without boundary except when stated otherwise. If $X$ is a semi-algebraic set in $\mathbb{R}^{n}$, by a semi-algebraic smooth submanifold in $X$ we mean a smooth submanifold of $\mathbb{R}^{n}$ that is a semi-algebraic subset of $X$.

Definition 2.2 A semi-algebraic set $X \subset \mathbb{R}^{N}$ is called bounded if it is contained in a ball of finite radius.

A fundamental tool in the theory of semi-algebraic sets we will need is the existence of certain type of finite partitions into smooth submanifolds called stratifications. By a smooth stratification of a subset $X$ of $\mathbb{R}^{n}$, we mean a finite partition $\mathcal{S}$ of $X$ such that each $S \in \mathcal{S}$ is a connected smooth submanifold and its relative closure $X \cap \bar{S}$ is a union of elements of $\mathcal{S}$, one being $S$ and the others being of $\operatorname{dimension}<\operatorname{dim} S$. Here an element of $\mathcal{S}$ is called a stratum, and $\mathcal{S}$ is called a semi-algebraic stratification if each of its strata is semi-algebraic. Semi-algebraic sets and mappings admit smooth semialgebraic stratifications. More precisely, we have the following [1, Proposition 9.1.8]:

Proposition 2.3 Given a semi-algebraic set $X \subset \mathbb{R}^{m}$ and a semi algebraic map $f: X \rightarrow \mathbb{R}^{n}$ there exists a stratification $\mathcal{S}$ of $X$ such that each stratum $S$ is a semialgebraic smooth submanifold, the restriction $\left.f\right|_{S}$ is a smooth map of constant rank, and $\{f(S): S \in \mathcal{S}\}$ is a stratification of $f(X)$.

We will call $\mathcal{S}$ of Proposition 2.3 a smooth semi-algebraic stratification of $X$ with respect to $f$. Given a finite family $\mathcal{A}$ of semi-algebraic subsets of a semi-algebraic set $X$ (for example $\mathcal{A}$ may itself be a stratification), we say that the stratification $\mathcal{S}$ is a refinement of $\mathcal{A}$ if each element of $\mathcal{A}$ is a union of strata in $\mathcal{S}$. The above smooth stratification of $X$ relative to $f$ can be chosen to be a refinement of any given finite family $\mathcal{A}$ of semi-algebraic subsets of $X$.

There is a larger class of subanalytical sets introduced by Hironaka in [14] (see also Hardt [10]) which he proved are also smoothly stratifiable.

If $T \subset \mathbb{R}^{N}$ is smoothly stratifiable, the local dimension of $T$ at $x \in T$, denoted by $\operatorname{dim}_{x}(T)$, is the highest dimension of a smooth submanifold $M \subset T$ such that $x \in \bar{M}$. The dimension of $T$ is $\operatorname{dim}(T)=\sup _{x \in T} \operatorname{dim}_{x} T$. If $T \subset X$ is an inclusion of smoothly stratifiable sets in $\mathbb{R}^{N}$, we say that $T$ is of codimension (at least) 1 in $X$ 
if, for each $x \in T, \operatorname{dim}_{x}(T)<\operatorname{dim}_{x}(X)$. For a semi-algebraic set $X$ and a map $f: X \rightarrow Y, \operatorname{dim}(\bar{X} \backslash X)<\operatorname{dim} X[1$, Theorem 2.8.12] and $\operatorname{dim} f(X) \leq \operatorname{dim} X[1$, Section 2].

For $k \geq 0$, any subset $Y$ of $\mathbb{R}^{n}$ has a $k$-dimensional Hausdorff (outer) measure, $\mathcal{H}^{k}(Y) \in[0, \infty]$, defined for example in [6, 2.10.2]. In particular, the $k$-dimensional Hausdorff measure of a $k$-dimensional smooth submanifold of $\mathbb{R}^{n}$ is its usual $k-$ dimensional volume with respect to the Riemannian metric induced from $\mathbb{R}^{n}$. For a smoothly stratifiable set $X \subset \mathbb{R}^{N}, \operatorname{dim}(X)<k$ if and only if $\mathcal{H}^{k}(X)=0$. For us, the key property will be that bounded semi-algebraic sets have finite volume.

Theorem 2.4 If $X$ is a bounded semi-algebraic set of dimension $\leq k$ then its Hausdorff $k$-measure $\mathcal{H}^{k}(X)$ is finite.

This fact was discovered in the complex case by Lelong [21] and is proved in the real case in Federer [6, 3.4.8 (13)]. See also van den Dries [5, Proposition 4.1, page 178] for a more modern reference.

Compact semi-algebraic sets admit semi-algebraic triangulations. More precisely, if $X$ is a compact semi-algebraic set, there exists a finite simplicial subcomplex $K$ of $\mathbb{R}^{q}$ for some $q$ and a semi-algebraic homeomorphism $\phi: K \stackrel{\cong}{\rightrightarrows} X$. Moreover, if $\mathcal{S}$ is a given stratification of $X$, we can choose $K$ and $\phi$ such that each stratum of $\mathcal{S}$ is the union of the images of some open simplices of $K$ under $\phi$ [1, Théorème 9.2.1]. The image under $\phi$ of the standard combinatorial stratification of $K$ into open simplices gives a new stratification that refines $\mathcal{S}$; this is called a triangulation of $X$ compatible with $\mathcal{S}$. Further, the polyhedron $K$ is unique up to PL homeomorphism (see Theorem 2.6). Any $n$-simplex can be naturally decomposed as a union of $n+1 n$-dimensional cubes, ie subspaces homeomorphic to $[0,1]^{n}$, whose vertices are the barycenters of the various faces of the simplex. These cubes meet along codimension 1 faces. Therefore any compact semi-algebraic set is semi-algebraically homeomorphic to a finite union of cubes; we call this a semi-algebraic cubification of the semi-algebraic set.

There is also a weaker notion of triangulation in the noncompact case. Indeed, since $\mathbb{R}^{n}$ is semi-algebraically homeomorphic to $(0,1)^{n}$, any semi-algebraic set is homeomorphic to a bounded one, so there is no loss of generality in assuming that a semi-algebraic set $X$ is bounded. Then its closure $\bar{X}$ is compact and admits a semi-algebraic triangulation that refines the stratification $\{X, \bar{X} \backslash X\}$. This means that $X$ is semi-algebraically homeomorphic to a finite union of open simplices and the collection of these simplices is a stratification. The closure in $X$ of each stratum consists of that open simplex and of all of its faces that belong to $X$. We call such an open simplex with some of its faces semi-open and this stratification a semi-open triangulation of $X$, or by abuse 
of terminology, a triangulation of $X$. Any finite stratification of $X$ can be refined by such a semi-open triangulation.

By the main result of [12] (see also [1, Proposition 9.3.1]), given a semi-algebraic map $f: X \rightarrow Y$ and a stratification of $X$ there exists a refinement of that stratification such that, for each stratum $S$ of this refinement, the restriction $\left.f\right|_{S}: S \rightarrow f(S)$ is a trivial bundle. In other words, there exists a semi-algebraic homeomorphism $S \cong f(S) \times F$ in which $\left.f\right|_{S}$ corresponds to the projection on the first factor. We say that the refined stratification trivializes $f$.

A stratification particularly useful to us is the following:

Lemma 2.5 Let $X \subset \mathbb{R}^{N}$ be a semi-algebraic set of dimension $k$ and let $f: X \rightarrow \mathbb{R}^{n}$ be a semi-algebraic map. Any stratification of $X$ can be refined to a stratification $\mathcal{S}$ with each of its strata $S$ a smooth submanifold (without boundary) of $\mathbb{R}^{N}$, and with the restriction $\left.f\right|_{S}$ a smooth map that satisfies:

- $\left.f\right|_{S}$ is of rank $k, f(S)$ is a submanifold of dimension $k$ in $\mathbb{R}^{n}$, and $\left.f\right|_{S}$ is a diffeomorphism between $S$ and $f(S)$; or

- $\left.f\right|_{S}$ is of rank $<k$ and $\operatorname{dim}(f(S))<k$.

This dichotomy is preserved by passing to a smooth refinement of $\mathcal{S}$. The stratification $\mathcal{S}$ can furthermore be refined so that for each pair of strata $S$ and $S^{\prime}$ on which the restriction of $f$ is of rank $k$, either $f(S)=f\left(S^{\prime}\right)$ or $\overline{f(S)} \cap f\left(S^{\prime}\right)=\varnothing$.

Proof Start with a stratification of $f(X)$ into smooth submanifolds and refine its preimage under $f$ into a smooth semi-algebraic stratification of $X$ with respect to $f$ as in Proposition 2.3. Take a refinement that trivializes $f$ and such that each stratum is connected. We still have that on each stratum $S$ of this refinement, $\left.f\right|_{S}$ is either of constant rank $k$ or everywhere of rank $<k$. Let $S$ be a stratum such that $\left.f\right|_{S}$ is of constant rank $k$. Since $\left.f\right|_{S}: S \rightarrow f(S)$ is a trivial bundle and its domain and codomain are both of dimension $k$, its fiber is discrete. Since $S$ is connected, we deduce that $\left.f\right|_{S}$ is a bijection. Moreover $f(S)$ is contained in a submanifold of dimension $k$, ie $\left.f\right|_{S}$ is a smooth injective immersion into a submanifold of the same dimension. Thus $f(S)$ is a submanifold of dimension $k$ and $\left.f\right|_{S}$ is a diffeomorphism onto its image. On the other hand, if $S$ is a stratum such that $\operatorname{rank}\left(\left.f\right|_{S}\right)<k$ then $\operatorname{dim} f(S)<k$.

It is clear that this dichotomy is preserved by passing to a refinement.

For the last part, let $S_{1}, \ldots, S_{l}$ be the strata on which $f$ is of rank $k$. Let $\mathcal{A}$ be the smallest partition of $\bigcup_{i=1}^{l} S_{i}$ such that each set $S_{i} \cap \bigcap_{j \in J} f^{-1}\left(f\left(S_{j}\right)\right)$ is a 
union of elements of $\mathcal{A}$ for $1 \leq i \leq l$ and $J \subset\{1, \ldots, l\}$. Consider the family $\mathcal{A}^{\prime}=\left\{\operatorname{int}_{X}(A): A \in \mathcal{A}\right\}$, where $\operatorname{int}_{X}(A)$ is the interior of $A$ in $X$. Then for $A_{1}, A_{2} \in \mathcal{A}^{\prime}$ we have that $f\left(A_{1}\right)=f\left(A_{2}\right)$ or $\overline{f\left(A_{1}\right)} \cap f\left(A_{2}\right)=\varnothing$. Moreover $\cup \mathcal{A}^{\prime}$ is dense in $\bigcup_{i=1}^{l} S_{i}$. Now let $X^{\prime}=X \backslash \cup \mathcal{A}^{\prime}$ (this is also semi-algebraic) and take a smooth semialgebraic stratification $\mathcal{S}^{\prime}$ of $X^{\prime}$ with respect to $\left.f\right|_{X^{\prime}}$ and that refines $\left\{\overline{A^{\prime}} \cap X^{\prime}: A^{\prime} \in \mathcal{A}^{\prime}\right\}$ It is straightforward to check that $\mathcal{A}^{\prime} \cup \mathcal{S}^{\prime}$ is a stratification of $X$ with the desired properties.

A maximal stratum is a stratum that is not a subset of the closure of any other stratum. The closure of a maximal stratum is disjoint from any other maximal stratum. The union of the maximal strata of a smooth stratification is a dense smooth submanifold (not necessarily of the same dimension on each connected component).

By partitioning each stratum into its connected components, we can refine any stratification into a stratification whose strata are connected. All the properties of stratifications considered above (smoothness, trivializations, properties from Lemma 2.5) are preserved by taking such connected refinements.

\subsection{Review of PL topology}

We will at several points throughout the paper need various results from PL topology. In particular we will need the Hauptvermutung (uniqueness of triangulations) for semialgebraic sets and the notion of collapsing polyhedra (strong form of contractibility). The basic reference from which we extract the following review of these concepts is Hudson [16, Chapters I-II] (most of the results are also in Rourke and Sanderson [22]).

A closed simplex in $\mathbb{R}^{n}$ is the convex hull of at most $n+1$ points in general position. An open simplex is the interior of a closed simplex in the affine subspace of the same dimension and containing the closed simplex (a point is both a closed and an open 0-simplex). A closed simplex can be partitioned into a finite family of open simplices called its faces. In this paper we define a polyhedron as a subspace of $\mathbb{R}^{n}$ that is the union of finitely many closed simplices (therefore in this paper a polyhedron is always compact). A PL map between two polyhedra is a continuous map whose graph is a polyhedron and it is a PL homeomorphism if it is bijective.

A simplicial complex is a finite partition of a polyhedron into open simplices, also called a triangulation of the polyhedron. A subdivided triangulation of a given triangulation is another triangulation which is a refinement (as partition) of the former. Any two triangulations of the same polyhedron admit a common subdivided triangulation. We will often abuse notation by identifying a simplicial complex (which is a partition) with 
its geometric realization (which is a polyhedron obtained by taking the union of the elements of the partition.)

A $P L$ ball is a polyhedron PL homeomorphic to a closed simplex. A subspace $F \subset B$ of a PL ball $B$ is called a face if there is a PL homeomorphism from $B$ to some simplex that sends $F$ onto a face of that simplex. If $P_{0} \subset P_{1}$ is an inclusion of polyhedra one says that $P_{1}$ elementary collapses to $P_{0}$ if $\overline{P_{1} \backslash P_{0}}$ is a ball and if $\overline{P_{1} \backslash P_{0}} \cap P_{0}$ is a face of that ball. More generally, a polyhedron $P$ collapses to a subpolyhedron $P_{0}$ if there is a decreasing sequence of polyhedra, $P=P_{r} \supset P_{r-1} \supset \ldots \supset P_{0}$, such that $P_{i}$ elementary collapses to $P_{i-1}, 1 \leq i \leq r$. In this situation we write $P \searrow P_{0}$. One says that a polyhedron $P$ is collapsible if $P \searrow *$, where $*$ is a one-point space. A collapsible polyhedron is clearly contractible but the converse is not true (a counterexample is given by the "dunce hat" of Zeeman [25]).

We say that a simplicial complex $K$ simplicially collapses to a subcomplex $K_{0}$, and we write $K \searrow^{s} K_{0}$, if there exists a sequence of simplicial subcomplexes $K=$ $K_{r} \supset K_{r-1} \supset \cdots \supset K_{0}$ such that $K_{i}=K_{i-1} \cup \tau_{i}$ where $\tau_{i}$ is a simplex with an open codimension 1 face which is disjoint form $K_{i-1}$ [16, Definition in II.1 and remark following it, page 43].

It is clear that if a simplicial complex simplicially collapses to a subcomplex, then there is a collapse of the underlying polyhedra. Conversely, suppose that a polyhedron $P$ collapses to a subpolyhedron $P_{0}$ and that $K$ is a triangulation of $P$ such that some subcomplex $K_{0}$ of $K$ is a triangulation of $P_{0}$. Then there exists a subdivision $K^{\prime}$ of $K$ that simplicially collapses to $K_{0}^{\prime}$ [16, Theorem 2.4]. In particular, any triangulation of a PL ball admits a subdivided triangulation that simplicially collapses to a point.

We have already reviewed the fact that a compact semi-algebraic set admits a semialgebraic triangulation, ie is semi-algebraically homeomorphic to a compact polyhedron. A very useful fact is the following result about the uniqueness of this triangulation, proved by Shiota and Yokoi [23, Corollary 4.3].

Theorem 2.6 (Hauptvermutung for compact semi-algebraic sets) If a compact semialgebraic set is semi-algebraically homeomorphic to two polyhedra, then those polyhedra are PL-homeomorphic.

The following is an immediate consequence.

Corollary 2.7 If a compact semi-algebraic set is semi-algebraically homeomorphic to a collapsible polyhedron, then any semi-algebraic triangulation of it admits a subdivided triangulation that simplicially collapses to a vertex. 


\subsection{Review of currents}

To define semi-algebraic chains, we will find the language of currents very convenient, so we review this notion here. A basic reference is Federer [6].

Denote by $\mathcal{D}^{k}\left(\mathbb{R}^{n}\right)$ the vector space of smooth differential $k$-forms on $\mathbb{R}^{n}$ with compact support. This space can be endowed with the with the usual Frechet topology (see $[6,4.1 .1]$ ) but we will not use it The exterior differential $d: \mathcal{D}^{k}\left(\mathbb{R}^{n}\right) \rightarrow \mathcal{D}^{k+1}\left(\mathbb{R}^{n}\right)$ is linear and continuous.

A $k$-current on $\mathbb{R}^{n}$ is a linear continuous form $T: \mathcal{D}^{k}\left(\mathbb{R}^{n}\right) \rightarrow \mathbb{R}$. We denote by $\mathcal{D}_{k}\left(\mathbb{R}^{n}\right)$ the vector space of $k$-currents. In other words, $\mathcal{D}_{k}\left(\mathbb{R}^{n}\right)$ is the topological dual of $\mathcal{D}^{k}\left(\mathbb{R}^{n}\right)$. The adjoint of the exterior differential $d: \mathcal{D}^{k}\left(\mathbb{R}^{n}\right) \rightarrow \mathcal{D}^{k+1}\left(\mathbb{R}^{n}\right)$ defines a boundary operator $\partial: \mathcal{D}_{k+1}\left(\mathbb{R}^{n}\right) \rightarrow \mathcal{D}_{k}\left(\mathbb{R}^{n}\right)$ by $\partial T(\omega)=T(d \omega)$.

For $U$ an open subset of $\mathbb{R}^{n}$, we define $\mathcal{D}^{k}(U)$ as the subspace of smooth forms in $\mathbb{R}^{n}$ whose support lies in $U$. The support of a current $T$ in $\mathbb{R}^{n}, \operatorname{spt}(T)$, is the smallest closed subset of $\mathbb{R}^{n}$ such that $\omega \in \mathcal{D}^{k}\left(\mathbb{R}^{n} \backslash \operatorname{spt}(T)\right) \Longrightarrow T(\omega)=0$. Clearly $\operatorname{spt}(\partial T) \subset \operatorname{spt}(T)$. For $X \subset \mathbb{R}^{n}$ we set

$$
\mathcal{D}_{k}(X)=\left\{T \in \mathcal{D}_{k}\left(\mathbb{R}^{n}\right): \operatorname{spt}(T) \subset X\right\}
$$

and thus get a chain complex

$$
\mathcal{D}_{*}(X):=\left(\bigoplus_{k \geq 0} \mathcal{D}_{k}(X), \partial\right) .
$$

The most important example of a current for us is the following:

Example 2.8 Denote by $\mathcal{H}^{k}$ the $k$-dimensional Hausdorff measure on $\mathbb{R}^{n}$. Let $V$ be a smooth $k$-dimensional oriented submanifold of $\mathbb{R}^{n}$ such that $\mathcal{H}^{k}(V)<\infty$. One defines a $k$-current $\llbracket V \rrbracket \in \mathcal{D}_{k}\left(\mathbb{R}^{n}\right)$ by

$$
\llbracket V \rrbracket(\omega)=\int_{V} \omega, \quad \omega \in \mathcal{D}^{k}\left(\mathbb{R}^{n}\right) .
$$

Currents can be equipped with various (semi-)norms and we review some of them from [6, Section 1.8.1 page 38, Section 4.1.7 page 358, and Section 4.1.12 pages 367-368].

\section{Definition 2.9}

- The comass of a differential form $\omega \in \mathcal{D}^{k}\left(\mathbb{R}^{n}\right)$ is

$$
\mathbf{M}(\omega)=\sup \left\{\omega(x)\left(v_{1} \wedge \cdots \wedge v_{k}\right): x \in \mathbb{R}^{n}, v_{i} \in \mathbb{R}^{n},\left\|v_{i}\right\| \leq 1\right\} \in \mathbb{R}_{+} .
$$


- The mass of a $k$-current $T \in \mathcal{D}_{k}\left(\mathbb{R}^{n}\right)$ is

$$
\mathbf{M}(T)=\sup \left\{T(\omega): \omega \in \mathcal{D}^{k}\left(\mathbb{R}^{n}\right), \mathbf{M}(\omega) \leq 1\right\} \in \mathbb{R}_{+} \cup\{\infty\} .
$$

- A current $T \in \mathcal{D}_{k}\left(\mathbb{R}^{n}\right)$ is called normal if $\operatorname{spt}(T)$ is compact and $\mathbf{M}(T)$ and $\mathbf{M}(\partial T)$ are finite.

- The flat seminorm of $T \in \mathcal{D}_{k}\left(\mathbb{R}^{n}\right)$ relative to a compact set $K \subset \mathbb{R}^{n}$ is defined by

$$
\mathbf{F}_{K}(T)=\inf \left\{\mathbf{M}(T-\partial S)+\mathbf{M}(S): S \in \mathcal{D}_{k+1}\left(\mathbb{R}^{n}\right), \operatorname{spt}(S) \subset K\right\} .
$$

- A current is called a flat chain if it is the limit of a sequence of normal currents for the flat seminorm relative to some compact set.

We will write $\mathbf{M}_{k}(T)$ for the mass when we want to emphasize the dimension of the current. Also, when there is no ambiguity about the compact set $K$ in the definition of the flat seminorm, we will drop it from the notation.

A normal current is clearly a flat chain. Also, the boundary of a flat chain is a flat chain [6, page 368]. There are two other properties of flat chains we will use:

(1) Absolute continuity, which states that if $T$ is a $k$-dimensional flat chain and if $\mathcal{H}^{k}(\operatorname{spt}(T))=0$, then $T=0$ [6, Theorem 4.1.20];

(2) Constancy theorem, which states that if $U$ is an open set in $\mathbb{R}^{n}, T$ is a flat $k$-chain in $\mathbb{R}^{n}$ with $U \cap \operatorname{spt}(T)$ a connected $k$-dimensional oriented smooth submanifold $V$ and $U \cap \operatorname{spt}(\partial T)=\varnothing$, then, in $U, T$ is just a constant multiple of the $k$-current $\llbracket V \rrbracket$ given by integration along $V$ [6, Section 4.1 .31 (2)]. In case $T$ is an integral flat chain (as will be the case for the semi-algebraic chains considered later), this multiple is an integer.

The norms of Definition 2.9 can be used to study convergence of sequences of currents. It is clear that convergence in mass implies convergence in the flat seminorm. We can also equip $\mathcal{D}_{k}\left(\mathbb{R}^{n}\right)$ with its weak topology characterized by the fact that a sequence of currents $\left(T_{n}\right)_{n \geq 1}$ converges weakly to $T$ in $\mathcal{D}_{k}\left(\mathbb{R}^{n}\right)$, which we denote by $T_{n} \rightarrow T$, if, for each $\omega \in \mathcal{D}^{k}\left(\mathbb{R}^{n}\right),\left(T_{n}(\omega)\right)_{n \geq 1}$ converges to $T(\omega)$ in $\mathbb{R}$. An elementary computation shows that convergence in flat norm implies weak convergence.

If $V$ is a smooth oriented submanifold of dimension $k$ in $\mathbb{R}^{n}$ with finite Hausdorff $k$-measure, then the current $\llbracket V \rrbracket: \omega \mapsto \int_{V} \omega$ is of mass $\mathbf{M}(\llbracket V \rrbracket)=\mathcal{H}^{k}(V)$. 


\section{Semi-algebraic chains}

The goal of this section is to functorially associate to each semi-algebraic set $X$ a chain subcomplex $\left(\mathrm{C}_{*}(X), \partial\right) \subset\left(\mathcal{D}_{*}(X), \partial\right)$ consisting of "semi-algebraic chains".

Let $M$ be an oriented compact semi-algebraic manifold of dimension $k$ and let $f: M \rightarrow \mathbb{R}^{n}$ be a semi-algebraic map. Consider a stratification of $M$ with connected strata, having all the properties of Lemma 2.5. Let $S_{1}, \ldots, S_{l}$ be the strata such that $\left.f\right|_{S_{i}}$ is of rank $k$. Each of the submanifolds $S_{i}$ is oriented by the orientation of $M$. Consider the family $\mathcal{N}=\left\{f\left(S_{i}\right): 1 \leq i \leq l\right\}$ of image submanifolds, which could be of cardinality $<l$ because $f\left(S_{i}\right)=f\left(S_{j}\right)$ for $i \neq j$ is a possibility. Fix an arbitrary orientation on each $N \in \mathcal{N}$ and set $n_{N}=\sum_{i, f\left(S_{i}\right)=N} \epsilon_{i}$ where $\epsilon_{i}= \pm 1$, the sign depending on whether the diffeomorphism $\left.f\right|_{S_{i}}: S_{i} \cong N$ preserves or reverses the orientation. Since $M$ is compact, the semi-algebraic set $N \subset f(M)$ is bounded and by Theorem 2.4 it is of finite $k$-volume. Therefore we can define a current $f_{*} \llbracket M \rrbracket \in \mathcal{D}_{k}\left(\mathbb{R}^{n}\right)$ by the formula

$$
f_{*} \llbracket M \rrbracket(\omega)=\sum_{N \in \mathcal{N}} n_{N} \int_{N} \omega
$$

for $\omega \in \mathcal{D}^{k}\left(\mathbb{R}^{n}\right)$. This current is of finite mass, in other words, $\mathbf{M}\left(f_{*} \llbracket M \rrbracket\right)=$ $\sum_{N \in \mathcal{N}}\left|n_{N}\right| \cdot \mathcal{H}^{k}(N)<\infty$. Notice also that

$$
f_{*} \llbracket M \rrbracket(\omega)=\sum_{i=1}^{l} \int_{S_{i}} f^{*} \omega .
$$

It is easy to check that the right side of the last equation does not depend on the choice of the stratification, because it is clearly unchanged under refinement, and any two stratifications satisfying the properties of Lemma 2.5 have a common refinement with the same properties. It is also clear that if $h: M^{\prime} \cong$ homeomorphism preserving orientation, then currents $f_{*}(\llbracket M \rrbracket)$ and $(f \circ h)_{*}\left(\llbracket M^{\prime} \rrbracket\right)$ are equal. Note that if $f$ is also smooth or even Lipschitz, then $f_{*}(\llbracket M \rrbracket)$ coincides with the usual pushforward operation for currents [6, Section 4]. However, a semi-algebraic map in general is not Lipschitz even though it is piecewise smooth.

Definition 3.1 A semi-algebraic $k$-chain in $\mathbb{R}^{n}$ is a current of the form $f_{*} \llbracket M \rrbracket \in$ $\mathcal{D}_{k}\left(\mathbb{R}^{n}\right)$ as constructed above for some oriented compact semi-algebraic $k$-dimensional manifold $M$ and some semi-algebraic map $f: M \rightarrow \mathbb{R}^{n}$. The set of semi-algebraic $k$-chains in $\mathbb{R}^{n}$ is denoted by $\mathrm{C}_{k}\left(\mathbb{R}^{n}\right)$. If $X$ is a semi-algebraic set in $\mathbb{R}^{n}$, we set

$$
\mathrm{C}_{k}(X)=\left\{\gamma \in \mathrm{C}_{k}\left(\mathbb{R}^{n}\right): \operatorname{spt}(\gamma) \subset X\right\} .
$$


The elements of $\mathrm{C}_{k}(X)$ are called the semi-algebraic $k$-chains in $X$, or simply $k$-chains.

Lemma 3.2 $\mathrm{C}_{k}(X)$ is a subgroup of $\mathcal{D}_{k}(X)$.

Proof The chain $f_{1 *} \llbracket M_{1} \rrbracket+f_{2 *} \llbracket M_{2} \rrbracket$ can be represented by $\left(f_{1} \sqcup f_{2}\right)_{*} \llbracket M_{1} \sqcup M_{2} \rrbracket$ where, without loss of generality, we have supposed that the manifolds $M_{1}$ and $M_{2}$ are disjoint subsets of the same $\mathbb{R}^{m}$. Also $-f_{*} \llbracket M \rrbracket=f_{*} \llbracket-M \rrbracket$ where $-M$ is $M$ with the opposite orientation.

The proof of the following is straightforward.

Proposition 3.3 If $i>\operatorname{dim}(X)$, then $\mathrm{C}_{i}(X)=0$.

It is clear that any semi-algebraic chain in $\mathrm{C}_{k}(X)$ can be represented by a semialgebraic map $g: M \rightarrow X$ where $M$ is an oriented compact semi-algebraic manifold of dimension $k$. However, here is another useful representation of semi-algebraic chains.

Proposition 3.4 (Alternative description of semi-algebraic chains) Let $V_{1}, \ldots, V_{r}$ be disjoint smooth semi-algebraic oriented $k$-dimensional submanifolds of $\mathbb{R}^{N}$ such that each $\bar{V}_{i}$ is a compact subset of the semi-algebraic set $X \subset \mathbb{R}^{N}$. Let $n_{1}, \ldots, n_{r}$ be integers. Then $\sum_{i=1}^{r} n_{i} \llbracket V_{i} \rrbracket$ represents an element of $\mathrm{C}_{k}(X)$. Conversely, any element of $\mathrm{C}_{k}(X)$ admits such a representation.

Proof Let $X$ be a semi-algebraic set and let $V \subset X$ be an oriented $k$-dimensional semi-algebraic manifold, not necessarily compact but with a compact closure $\bar{V}$ in $X$. We do not suppose that $\bar{V}$ is a manifold. We can stratify $V$ into a finite number of disjoint smooth semi-algebraic submanifolds $V_{i}$ of dimension $k$ and a subset $Z$ of dimension $<k$. By Theorem 2.4, the $V_{i}$ have finite $k$-volume and we can define the pushforward current $\llbracket V \rrbracket \in \mathcal{D}_{k}(X)$ by

$$
\llbracket V \rrbracket(\omega)=\sum_{i} \int_{V_{i}} \omega .
$$

This formula is independent of the choice of stratification. We want to show that $\llbracket V \rrbracket \in \mathrm{C}_{k}(X)$. Notice first that by [1, Proposition 2.8.12], $\operatorname{dim}(\bar{V} \backslash V)<k$ and $\operatorname{dim}\left(\bar{V}_{i} \backslash V_{i}\right)<k$. The compact space $\bar{V}$ admits a semi-algebraic triangulation $\phi: K \stackrel{\cong}{\rightrightarrows} \bar{V}$ where $K$ is a finite simplicial complex and we can assume that the triangulation refines the family $\left\{V_{i}\right\} \cup\{Z\}$. Each closed $k$-dimensional simplex $\sigma$ 
of $K$ is mapped into the closure of some $V_{i}$ and the orientation of $V_{i}$ induces an orientation of $\sigma$. We thus get a semi-algebraic $k$-chain $\phi_{*} \llbracket \sigma \rrbracket$ and it is clear that $\llbracket V \rrbracket=\sum_{\sigma} \phi_{*} \llbracket \sigma \rrbracket \in \mathrm{C}_{k}(X)$, where the sum is taken over all $k$-simplices of $K$.

The converse is an immediate consequence of formula (1).

We now turn to functorial properties of semi-algebraic chains. Let $g: X \rightarrow Y$ be a semi-algebraic map. If $f_{*} \llbracket M \rrbracket$ is a semi-algebraic chain in $X$ then $(g \circ f)_{*} \llbracket M \rrbracket=$ $g_{*}\left(f_{*} \llbracket M \rrbracket\right)$ is a semi-algebraic chain in $Y$. It is easy to check that this gives a well-defined homomorphism

$$
g_{*}: \mathrm{C}_{k}(X) \longrightarrow \mathrm{C}_{k}(Y)
$$

and that this association is functorial. In other words, $(h \circ g)_{*}=h_{*} \circ g_{*}$ and $\mathrm{id}_{*}=\mathrm{id}$. In order to prove that $g_{*}$ defines a chain map in Corollary 3.7, we need the following.

Theorem 3.5 Let $M$ be a compact semi-algebraic oriented manifold and $f: M \rightarrow \mathbb{R}^{n}$ be a semi-algebraic map. Then $\partial\left(f_{*}(\llbracket M \rrbracket)\right)=f_{*}(\llbracket \partial M \rrbracket)$.

Remark 3.6 When $f$ is the identity map, this theorem can be thought of as a generalization of Stokes' Theorem which states that $\partial \llbracket M \rrbracket=\llbracket \partial M \rrbracket$, and in the general case it is a combination of Stokes' Theorem and naturality of the boundary operator with respect to $f_{*}$.

Proof Suppose that $M \subset \mathbb{R}^{m}$ and set $k=\operatorname{dim} M$. Take a triangulation of $M$ such that each stratum $S$ is a smooth submanifold of $\mathbb{R}^{m}$ and $\left.f\right|_{S}$ is smooth. Since both sides of the equation we want to establish are clearly additive, it is enough to prove that the equation holds on each closed simplex of maximal dimension in that triangulation. Thus we can assume that $M$ is stratified as the standard combinatorial stratification of the $k$-simplex $\Delta^{k}$. In particular, $M$ has the unique maximal stratum $V=\operatorname{int}(\mathrm{M})=M \backslash \partial M$ of dimension $k$, and $k+1$ codimension 1 connected strata $S_{0}, \ldots, S_{k}$ whose union is dense in $\partial M$. Since $f$ is smooth on the interior of the simplex [1, Remark 9.2.3], by replacing the original triangulation by its barycentric subdivision we can furthermore assume that $\left.f\right|_{M \backslash \overline{S_{0}}}$ is smooth.

The proof now proceeds by induction on $k=\operatorname{dim}(M)$. If $k=0$, there is nothing to show. If $k=1$, there exists a semi-algebraic homeomorphism $\phi:[0,1] \stackrel{\cong}{\rightrightarrows} M$ that is smooth on $(0,1]$. For $0<\epsilon<1$, set $M_{\epsilon}=\phi([\epsilon, 1])$. Since $\mathbf{M}\left(f_{*} \llbracket M \rrbracket\right)<\infty$, Lebesgue's Bounded Convergence Theorem and smooth Stokes' Theorem imply that 
for each smooth 0 -form $\omega \in \mathcal{D}^{0}\left(\mathbb{R}^{n}\right)$ we have

$$
\begin{aligned}
& \left(\partial f_{*} \llbracket M \rrbracket\right)(\omega) \\
& =f_{*} \llbracket M \rrbracket(d \omega)=\int_{\operatorname{int}(M)} f^{*}(d \omega)=\lim _{\epsilon \rightarrow 0} \int_{M_{\epsilon}} f^{*}(d \omega)=\lim _{\epsilon \rightarrow 0} \int_{\partial M_{\epsilon}} f^{*}(\omega) \\
& =\lim _{\epsilon \rightarrow 0} \omega(f(\phi(1)))-\omega(f(\phi(\epsilon)))=\omega(f(\phi(1)))-\omega(f(\phi(0)))=\left(f_{*}[\partial M]\right)(\omega) .
\end{aligned}
$$

This proves the result for $k=1$.

Suppose that the theorem has been proved in dimension $<k$ for some $k \geq 2$. We first prove it in dimension $k$ with the assumption that $f$ is injective on $M$ and of maximal rank on each stratum.

To do this, we first observe that $f_{*} \llbracket S_{i} \rrbracket$ and $\partial f_{*} \llbracket M \rrbracket$ are flat chains as in Definition 2.9. Notice that $f_{*} \llbracket S_{i} \rrbracket=f_{*} \llbracket \overline{S_{i}} \rrbracket$ and, by induction hypothesis, $\partial f_{*} \llbracket \overline{S_{i}} \rrbracket=f_{*} \llbracket \partial \overline{S_{i}} \rrbracket$. Since these semi-algebraic chains are of finite mass we deduce that $f_{*} \llbracket S_{i} \rrbracket$ is a normal current, hence a flat chain. For $\llbracket M \rrbracket$, consider an increasing sequence $M_{1} \subset M_{2} \subset \cdots$ of $k$-dimensional compact smooth manifolds whose union is $V=\operatorname{int}(M)$. By the smooth Stokes' Theorem, $\mathbf{M}\left(\partial f_{*}\left[M_{n}\right]\right)=\mathbf{M}\left(f_{*}\left[\partial M_{n}\right]\right)<\infty$, and $\mathbf{M}\left(f_{*}\left[M_{n}\right]\right) \leq$ $\mathbf{M}\left(f_{*} \llbracket M \rrbracket\right)<\infty$. Thus each current $f_{*}\left[M_{n}\right]$ is normal and, by Lebesgue's convergence theorem, their sequence converges in mass, and hence in flat semi-norm, to $f_{*} \llbracket M \rrbracket$. This implies that $f_{*} \llbracket M \rrbracket$ is a flat chain, so the same is true for $\partial f_{*} \llbracket M \rrbracket$ by [6, page 368].

Using the fact that $f$ is smooth on $M \backslash \partial M$, and injective and continuous on $M$, it is easy to check with the smooth Stokes' Theorem that $\operatorname{spt}\left(\partial f_{*}(\llbracket M \rrbracket)\right) \subset f(\partial M)$.

To continue, we will use the constancy theorem from Section 2.3. Since $f$ is a homeomorphism on its image and is smooth on each stratum, $\bigcup_{i=0}^{k} f\left(S_{i}\right)$ is a smooth submanifold and is open in the closed subset $f(\partial M)$. Set $Z=f(\partial M) \backslash \bigcup_{i=0}^{k} f\left(S_{i}\right)$. This is a closed subset of dimension $<k-1$. Since each $f\left(S_{i}\right)$ is a connected component of $\bigcup_{i=0}^{k} f\left(S_{i}\right)$, by [6, Section 4.1.31 (2)] with $C=f\left(S_{i}\right)$ and $r=c_{i}$, there exist $c_{i} \in \mathbb{R}$ such that

$$
\operatorname{spt}\left(\partial f_{*}(\llbracket M \rrbracket)-c_{i} f_{*} \llbracket S_{i} \rrbracket\right) \cap f\left(S_{i}\right)=\varnothing .
$$

Now set $T=\partial f_{*}(\llbracket M \rrbracket)-\sum_{i=0}^{k} c_{i} f_{*}\left[S_{i}\right]$. We deduce that $\operatorname{spt}(T) \subset Z$. Since $T$ is a flat $(k-1)$-chain and $\operatorname{dim}(Z)<k-1$, Theorem 4.1 .20 of [6] implies that $T=0$. In other words,

$$
\partial f_{*}(\llbracket M \rrbracket)=\sum_{i=0}^{k} c_{i} f_{*} \llbracket S_{i} \rrbracket .
$$


Clearly $f_{*}[\partial M]=\sum_{i=0}^{k} f_{*} \llbracket S_{i} \rrbracket$, so it remains only to prove that all the $c_{i}$ 's are 1 . For $1 \leq i \leq k$, this is an immediate consequence of the classical Stokes' Theorem since $f$ is smooth on $M \backslash \overline{S_{0}}$. Applying the boundary operator to both sides of Equation (2) and using the induction hypothesis we get that

$$
c_{0} f_{*}\left[\partial \overline{S_{0}}\right]+\sum_{i=1}^{k} f_{*}\left[\partial \overline{S_{i}}\right]=0 .
$$

For $1 \leq i \leq k$, let $B_{i}$ be the $(k-2)$-dimensional stratum of $M$ whose closure is $\partial \overline{S_{0}} \cap \partial \overline{S_{i}}$. After canceling terms, the last equation becomes $\sum_{i=1}^{k}\left(c_{0}-1\right) f_{*}\left[B_{i}\right]=0$. Clearly $f\left(B_{1}\right) \subset \operatorname{spt}\left(f_{*}\left[B_{1}\right]\right)$ and, for $2 \leq i \leq k, f\left(B_{1}\right) \cap \operatorname{spt}\left(f_{*}\left[B_{i}\right]\right)=\varnothing$. Since $k \geq 2, f\left(B_{1}\right) \neq \varnothing$ and we deduce that $c_{0}=1$. This proves the theorem in dimension $k$ under the extra assumption that $f$ is injective and of maximal rank on each stratum.

For the general case, consider the semi-algebraic inclusion $i: M \hookrightarrow \mathbb{R}^{m}$ and, for $\epsilon \in[0,1]$, define the map

$$
\begin{aligned}
f_{\epsilon}: M & \longrightarrow \mathbb{R}^{n} \times \mathbb{R}^{m} \\
x & \longmapsto(f(x), \epsilon \cdot i(x)) .
\end{aligned}
$$

When $\epsilon>0, f_{\epsilon}$ is injective and smooth of maximal rank on each stratum, and therefore by the beginning of the proof we have that $\partial f_{\epsilon *}(\llbracket M \rrbracket)=f_{\epsilon *}(\llbracket \partial M \rrbracket)$. Since the boundary operator $\partial$ is continuous with respect to weak convergence, in order to finish the proof it is enough to show that $f_{\epsilon *}(\llbracket M \rrbracket)$ and $f_{\epsilon *}(\llbracket \partial M \rrbracket)$ converge weakly to $f_{0 *}(\llbracket M \rrbracket)$ and $f_{0 *}(\llbracket \partial M \rrbracket)$ as $\epsilon \rightarrow 0$, respectively.

We prove that $f_{\epsilon *}(\llbracket M \rrbracket) \rightarrow f_{0 *}(\llbracket M \rrbracket)$, the case of $\partial M$ being completely analogous. Let $\delta>0$. Consider a differential form $\alpha \in \mathcal{D}^{k}\left(\mathbb{R}^{n} \times \mathbb{R}^{m}\right)$. Since $f$ and $i$ are smooth on the complement of a codimension 1 semi-algebraic subset of $M$, there exists a compact semi-algebraic codimension 0 smooth submanifold with boundary $M^{\prime} \subset M$ on which $f$ and $i$ are smooth and such that $\mathbf{M}\left(f_{1 *}\left(\llbracket M \backslash M^{\prime} \rrbracket\right)\right) \leq \delta /(4 \cdot \mathbf{M}(\alpha))$. Moreover, notice that $f_{\epsilon}=q_{\epsilon} \circ f_{1}$, where $q_{\epsilon}$ is the map

$$
\begin{aligned}
q_{\epsilon}: \mathbb{R}^{n} \times \mathbb{R}^{m} & \longrightarrow \mathbb{R}^{n} \times \mathbb{R}^{m} \\
(u, v) & \longmapsto(u, \epsilon \cdot v)
\end{aligned}
$$

and which admits Lipschitz constant 1 . Therefore for all $\epsilon \in[0,1]$ we also have

$$
\mathbf{M}\left(f_{\epsilon *}\left(\llbracket M \backslash M^{\prime} \rrbracket\right)\right) \leq \frac{\delta}{4 \cdot \mathbf{M}(\alpha)} .
$$


Hence

$$
\left|\left\langle f_{\epsilon *}(\llbracket M \rrbracket)-f_{0 *}(\llbracket M \rrbracket), \alpha\right\rangle\right|
$$

$$
\begin{aligned}
& \leq\left|\left\langle f_{\epsilon *}\left(\llbracket M \backslash M^{\prime} \rrbracket\right)-f_{0 *}\left(\llbracket M \backslash M^{\prime} \rrbracket\right), \alpha\right\rangle\right|+\left|\left\langle f_{\epsilon *}\left(\llbracket M^{\prime} \rrbracket\right)-f_{0 *}\left(\llbracket M^{\prime} \rrbracket\right), \alpha\right\rangle\right| \\
& \leq \delta / 2+\left|\left\langle\llbracket M^{\prime} \rrbracket,\left(\left(\left.f_{\epsilon}\right|_{M^{\prime}}\right)^{*}-\left(\left.f_{0}\right|_{M^{\prime}}\right)^{*}\right)(\alpha)\right\rangle\right| .
\end{aligned}
$$

It is clear that $\left.f_{\epsilon}\right|_{M^{\prime}}$ converges in the $\mathcal{C}^{1}$-norm to $\left.f_{0}\right|_{M^{\prime}}$, and therefore for $\epsilon$ small enough the second summand in (3) is also less than $\delta / 2$. Therefore $f_{\epsilon *}(\llbracket M \rrbracket)$ converges weakly to $f_{0 *}(\llbracket M \rrbracket)$.

The proof that $f_{\epsilon *}(\llbracket \partial M \rrbracket) \rightarrow f_{0 *}(\llbracket \partial M \rrbracket)$ is exactly the same and we will omit it.

The following is now immediate.

\section{Corollary 3.7}

- If $X$ is a semi-algebraic set, then $\partial\left(\mathrm{C}_{k}(X)\right) \subset \mathrm{C}_{k-1}(X)$ and $\mathrm{C}_{*}(X):=$ $\left(\bigoplus_{k \geq 0} \mathrm{C}_{k}(X), \partial\right)$ is a chain complex.

- If $g: X \rightarrow Y$ is a semi-algebraic map, then $g_{*}: \mathrm{C}_{*}(X) \rightarrow \mathrm{C}_{*}(Y)$ is a chain map, that is, $g_{*} \partial=\partial g_{*}$.

We end this section by introducing a cross product on chains by the following easy result.

Proposition 3.8 Let $X_{1}$ and $X_{2}$ be semi-algebraic sets. There exists a degreepreserving linear map

$$
\times: \mathrm{C}_{*}\left(X_{1}\right) \otimes \mathrm{C}_{*}\left(X_{2}\right) \longrightarrow \mathrm{C}_{*}\left(X_{1} \times X_{2}\right)
$$

characterized by the formula

$$
f_{1 *}\left(\llbracket M_{1} \rrbracket\right) \times f_{2 *}\left(\llbracket M_{2} \rrbracket\right)=\left(f_{1} \times f_{2}\right)_{*}\left(\llbracket M_{1} \times M_{2} \rrbracket\right)
$$

where $M_{i}$ are compact oriented semi-algebraic manifolds and $f_{i}: M_{i} \rightarrow X_{i}$ are semialgebraic maps, $i=1,2$.

This product satisfies the Leibniz formula

$$
\partial\left(\gamma_{1} \times \gamma_{2}\right)=\partial\left(\gamma_{1}\right) \times \gamma_{2}+(-1)^{\operatorname{deg}\left(\gamma_{1}\right)} \gamma_{1} \times \partial\left(\gamma_{2}\right) .
$$

Let $T: X_{1} \times X_{2} \rightarrow X_{2} \times X_{1}$ given by $\left(x_{1}, x_{2}\right) \mapsto\left(x_{2}, x_{1}\right)$ be the twisting map. Then

$$
T_{*}\left(\gamma_{1} \times \gamma_{2}\right)=(-1)^{\operatorname{deg}\left(\gamma_{1}\right) \operatorname{deg}\left(\gamma_{2}\right)} \gamma_{2} \times \gamma_{1} \text {. }
$$




\section{Convergence in $\mathrm{C}_{k}(X)$}

The goal of this section is to introduce a suitable notion of convergence of sequences of semi-algebraic chains. There are three classical notions of convergence for currents, which are, from the strongest to the weakest (see Definition 2.9):

- convergence in mass (ie for the mass norm $\mathbf{M}$ );

- convergence in flat norm (ie for the norm $\mathbf{F}$ relative to some compact subspace);

- weak convergence, ie $T_{n} \rightarrow T$ in $\mathcal{D}_{k}\left(\mathbb{R}^{N}\right)$ if, for each $\omega \in \mathcal{D}^{k}\left(\mathbb{R}^{N}\right), T_{n}(\omega) \rightarrow$ $T(\omega)$ in $\mathbb{R}$.

However, these definitions of convergence cannot be adapted well to semi-algebraic chains because they are not preserved by semi-algebraic maps (because those are not locally Lipschitzian). As a simple example, consider the semi-algebraic map $f: \mathbb{R} \rightarrow \mathbb{R}, x \mapsto \sqrt{|x|}$. For $n \geq 1$ set $\gamma_{n}=n \cdot \llbracket\left[0,1 / n^{2}\right] \rrbracket$, which is a sequence in $\mathbf{C}_{1}(\mathbb{R})$. Then $\mathbf{M}\left(\gamma_{n}\right)=1 / n$ which converges to zero but $f_{*}\left(\gamma_{n}\right)=n \cdot \llbracket[0,1 / n] \rrbracket$ does not converge to 0 even weakly.

We thus introduce the following more suitable notion of convergence in $\mathrm{C}_{k}(X)$.

Definition 4.1 A sequence $\left(\gamma_{n}\right)_{n \geq 1}$ in $\mathrm{C}_{k}(X)$ converges semi-algebraically to $\gamma \in$ $\mathrm{C}_{k}(X)$, denoted by $\gamma_{n} \stackrel{\text { SA }}{\rightarrow} \gamma$, if there exists a semi-algebraic map $h: M \times[0,1] \rightarrow X$, where $M$ is a compact semi-algebraic oriented manifold, and a sequence $\left(\epsilon_{n}\right)_{n \geq 1}$ in $[0,1]$ converging to zero, such that $\gamma_{n}=h_{*}\left(\llbracket M \times\left\{\epsilon_{n}\right\} \rrbracket\right)$ and $\gamma=h_{*}(\llbracket M \times\{0\} \rrbracket)$.

The following is immediate from the definition.

Proposition 4.2 Let $f: X \rightarrow Y$ be a semi-algebraic map. If $\gamma_{n} \stackrel{\text { SA }}{\rightarrow} \gamma$ in $\mathrm{C}_{k}(X)$ then $f_{*}\left(\gamma_{n}\right) \stackrel{\text { SA }}{\rightarrow} f_{*}(\gamma)$ in $\mathrm{C}_{k}(Y)$.

The following says that if a semi-algebraic subset $X_{0}$ is dense in $X$, then $\mathrm{C}_{k}\left(X_{0}\right)$ is "dense" in $\mathrm{C}_{k}(X)$ in terms of SA convergence.

Proposition 4.3 Let $X$ be a semi-algebraic set and let $X_{0} \subset X$ be a dense semialgebraic subset. Then for each $\gamma \in \mathrm{C}_{k}(X)$, there exists a sequence $\left(\gamma_{n}\right)_{n \geq 1}$ in $\mathrm{C}_{k}\left(X_{0}\right)$ such that $\gamma_{n} \stackrel{\text { SA }}{\rightarrow} \gamma$.

Proof Choose a triangulation of $X$ such that $X_{0}$ and $X \backslash X_{0}$ are unions of simplices and such that $\gamma=\sum_{\sigma} n_{\sigma} \cdot \llbracket \bar{\sigma} \rrbracket$ where the sum runs over some $k$-simplices of the triangulation and the orientations of those simplices are chosen so that the integers $n_{\sigma}$ 
are nonnegative. For each such simplex, since $\sigma \subset \overline{X_{0}}$ and since $X_{0}$ is a union of simplices, there exists an open simplex $\tau$ (which could be $\sigma$ ) such that $\tau \subset X_{0}$ and $\sigma$ is a face of $\tau$. It is easy to build a semi-algebraic map

$$
h_{\sigma}: \Delta^{k} \times[0,1] \longrightarrow \bar{\tau}
$$

such that $h_{\sigma}\left(\Delta^{k} \times(0,1]\right) \subset \tau$ and $h_{\sigma}(-, 0): \Delta^{k} \cong \bar{\sigma}$ is a homeomorphism preserving the orientation. Set $M=\bigsqcup_{\sigma} \bigsqcup_{i=1}^{n_{\sigma}} \Delta^{k}$ and consider the map

$$
h=\bigsqcup_{\sigma} \bigsqcup_{i=1}^{n_{\sigma}} h_{\sigma}: M \times[0,1] \longrightarrow X .
$$

Then $\gamma_{n}=h_{*}(\llbracket M \times\{1 / n\} \rrbracket)$ is the desired sequence.

Our last important result is that SA (semi-algebraic) convergence implies weak convergence as currents.

Proposition 4.4 If $\gamma_{n} \stackrel{\text { SA }}{\rightarrow} \gamma$ in $\mathrm{C}_{k}(X)$ then $\gamma_{n} \rightarrow \gamma$.

Proof Take $h, M$, and $\epsilon_{n}$ as in Definition 4.1. Then

$$
\begin{aligned}
\gamma_{n}-\gamma & =h_{*}\left(\llbracket M \times \partial\left[0, \epsilon_{n}\right] \rrbracket\right) \\
& = \pm\left(h_{*}\left(\llbracket \partial\left(M \times\left[0, \epsilon_{n}\right]\right) \rrbracket\right)-h_{*}\left(\llbracket(\partial M) \times\left[0, \epsilon_{n}\right] \rrbracket\right)\right) \\
& = \pm\left(\partial h_{*}\left(\llbracket M \times\left[0, \epsilon_{n}\right] \rrbracket\right)-h_{*}\left(\llbracket(\partial M) \times\left[0, \epsilon_{n}\right] \rrbracket\right)\right)
\end{aligned}
$$

which implies that

$$
\mathbf{F}\left(\gamma_{n}-\gamma\right) \leq \mathbf{M}_{k+1}\left(h_{*}\left(\llbracket M \times\left[0, \epsilon_{n}\right] \rrbracket\right)\right)+\mathbf{M}_{k}\left(h_{*}\left(\llbracket(\partial M) \times\left[0, \epsilon_{n}\right] \rrbracket\right)\right)
$$

where the flat norm is taken with respect to the compact space $h(M \times[0,1])$.

Define the map

$$
\begin{aligned}
\tilde{h}: M \times[0,1] & \longrightarrow X \times M \\
(u, t) & \longmapsto(h(u, t), u) .
\end{aligned}
$$

Hence $\tilde{h}$ is a homeomorphism on its image and $h=\operatorname{pr}_{1} \tilde{h}$ where $\operatorname{pr}_{1}: X \times M \rightarrow X$ is the projection which is 1 -Lipschitzian. Therefore

$$
\begin{aligned}
\mathbf{M}_{k+1}\left(h_{*}\left(\llbracket M \times\left[0, \epsilon_{n}\right] \rrbracket\right)\right) & \leq \mathbf{M}_{k+1}\left(\tilde{h}_{*}\left(\llbracket M \times\left[0, \epsilon_{n} \rrbracket \rrbracket\right)\right)\right. \\
& =\mathbf{M}_{k+1}\left(\llbracket \tilde{h}\left(M \times\left[0, \epsilon_{n}\right]\right) \rrbracket\right) \\
& =\mathcal{H}^{k+1}\left(\tilde{h}\left(M \times\left[0, \epsilon_{n}\right]\right)\right) .
\end{aligned}
$$


By Theorem 2.4, $\mathcal{H}^{k+1}(\tilde{h}(M \times[0,1]))<\infty$. The Lebesgue Bounded Convergence Theorem implies that

$$
\mathcal{H}^{k+1}\left(\tilde{h}\left(M \times\left[0, \epsilon_{n}\right]\right) \rightarrow \mathcal{H}^{k+1}(\tilde{h}(M \times\{0\})) \text { as } n \rightarrow \infty\right.
$$

and $\mathcal{H}^{k+1}(\tilde{h}(M \times\{0\}))=0$ because $\operatorname{dim} \tilde{h}(M \times\{0\}) \leq k$. This implies that

$$
\lim _{n \rightarrow \infty} \mathbf{M}_{k+1}\left(h_{*}\left(\llbracket M \times\left[0, \epsilon_{n}\right] \rrbracket\right)\right)=0 .
$$

A completely analogous argument shows that

$$
\lim _{n \rightarrow \infty} \mathbf{M}_{k}\left(h_{*}\left(\llbracket(\partial M) \times\left[0, \epsilon_{n}\right] \rrbracket\right)\right)=0 .
$$

Thus $\lim _{n \rightarrow \infty} \mathbf{F}\left(\gamma_{n}-\gamma\right)=0$ which implies the weak convergence.

Remark 4.5 Actually, the above proof shows that SA-convergence implies convergence in the flat norm, which is stronger than weak convergence, but we will not use this fact.

\section{PA forms}

The aim of this section is to construct the contravariant functor of PA forms,

$$
\Omega_{\mathrm{PA}}^{*}: \text { SemiAlg } \rightarrow \text { CDGA, }
$$

(see Theorem 5.32), which we will show in Section 6 to be weakly equivalent to $A_{P L}(-; \mathbb{R})$. The construction is in two stages. We will first build a functor $\Omega_{\text {min }}^{*}$ of minimal forms in Section 5.2. Unfortunately this functor is not weakly equivalent to $A_{P L}(-; \mathbb{R})$ because it does not satisfy the Poincaré Lemma. Building on $\Omega_{\text {min }}^{*}$, we will then define $\Omega_{\mathrm{PA}}^{*}$ in Section 5.4 which will resolve this issue. These PA forms are defined by integration of minimal forms along the fiber, but to define this integration correctly, we will need the notion of strongly continuous family of chains which is given in Section 5.3.

\subsection{Semi-algebraic cochains and smooth forms}

Let $X$ be a semi-algebraic set. We consider the vector space of semi-algebraic cochains with values in $\mathbb{R}$ as the linear dual of the chains, ie we let

$$
\mathrm{C}^{k}(X)=\operatorname{hom}\left(\mathrm{C}_{k}(X), \mathbb{R}\right) .
$$

This gives a cochain complex of real vector spaces $C^{*}(X):=\bigoplus_{k \geq 0} C^{k}(X)$ with coboundary $\delta: \mathrm{C}^{k}(X) \rightarrow \mathrm{C}^{k+1}(X)$ defined as the adjoint of the boundary $\partial: \mathrm{C}_{k+1}(X) \rightarrow$ $\mathrm{C}_{k}(X)$. 
The real number giving the value of a cochain $\lambda \in \mathrm{C}^{k}(X)$ on a chain $\gamma \in \mathrm{C}_{k}(X)$ is denoted by $\langle\lambda, \gamma\rangle$. By convention this value is 0 if $\lambda$ and $\gamma$ have different degree.

One has a contravariant functor $\mathrm{C}^{*}: \operatorname{SemiAlg} \rightarrow \mathrm{Ch}^{*}(\mathbb{R})$ with values in cochain complexes over $\mathbb{R}$. In particular a semi-algebraic map $f: X \rightarrow Y$ induces a map of cochains $f^{*}: \mathrm{C}^{*}(Y) \rightarrow \mathrm{C}^{*}(X)$ defined by $\left\langle f^{*}(\lambda), \gamma\right\rangle=\left\langle\lambda, f_{*}(\gamma)\right\rangle$. When $f: X \hookrightarrow Y$ is an inclusion we write $\left.\lambda\right|_{X}:=f^{*}(\lambda)$.

Lemma 5.1 Let $W \subset \mathbb{R}^{N}$ be a semi-algebraic smooth submanifold of dimension $m$ and let $\omega \in \Omega_{\mathcal{C}}^{k}(W)$ be a smooth differential form. There is a well defined linear map

$$
\begin{aligned}
\langle\omega,-\rangle: \mathrm{C}_{k}(W) & \longrightarrow \mathbb{R} \\
\gamma & \longmapsto\langle\omega, \gamma\rangle=\sum n_{i} \int_{V_{i}} \omega
\end{aligned}
$$

when $V_{i}$ are bounded semi-algebraic smooth oriented submanifolds in $W$ and $n_{i}$ are integers such that $\gamma=\sum n_{i} \llbracket V_{i} \rrbracket$. Moreover, the map

$$
\begin{aligned}
\langle-,-\rangle: \Omega_{\mathcal{C}^{\infty}}^{*}(W) & \hookrightarrow \mathrm{C}^{*}(W) \\
\omega & \mapsto\langle\omega,-\rangle
\end{aligned}
$$

is an inclusion.

Proof By Proposition 3.4, a chain $\gamma \in \mathrm{C}_{k}(W)$ can be represented by a linear combination of smooth submanifolds $V_{i} \subset W$ of finite volume. Further, the smooth form $\omega$ is bounded on the compact support of $\gamma$. Therefore each integral $\int_{V_{i}} \omega$ converges and it is clear that the value of the linear combination depends only on the chain $\gamma$.

For the injectivity of the map (4) we need to show that the value of $\omega \in \Omega_{\mathcal{C}^{\infty}}^{k}(W)$ at any point $x \in W$ is completely characterized by the values of $\langle\omega,-\rangle$ on semi-algebraic chains in $W$. Indeed consider the $m$-dimensional affine subspace $T_{x} W \subset \mathbb{R}^{N}$ tangent to $W$ at $x$. The orthogonal projection $\pi$ of a neighborhood of $x$ in $W$ onto $T_{x} W$ is a semi-algebraic diffeomorphism onto its image. For an orthonormal $k$-multivector $\xi$ in $T_{x} W$ and a small $\epsilon>0$, let $\xi[\epsilon]$ be a $k$-dimensional cube in $T_{x} W$ based at $x$ with edges of length $\epsilon$ in the directions of $\xi$. Then $\llbracket \pi^{-1}(\xi[\epsilon]) \rrbracket \in \mathrm{C}_{k}(W)$ and the value of $\omega(\xi)$ is given by

$$
\lim _{\epsilon \rightarrow 0} \epsilon^{-k}\left\langle\omega, \llbracket \pi^{-1}(\xi[\epsilon]) \rrbracket\right\rangle .
$$

Definition 5.2 Let $W$ be a semi-algebraic smooth submanifold of $\mathbb{R}^{N}$. A cochain in $\mathrm{C}^{*}(W)$ which is in the image of $\Omega_{\mathcal{C}^{\infty}}^{*}(W)$ under the map (4) is called smooth. 
Lemma 5.3 (Strong Künneth formula for smooth cochains) Let $W_{1}$ and $W_{2}$ be two semi-algebraic smooth submanifolds in $\mathbb{R}^{n_{1}}$ and $\mathbb{R}^{n_{2}}$ and let $\omega \in \Omega_{\mathcal{C}}^{*}\left(W_{1} \times W_{2}\right)$. If, for all $\gamma_{1} \in \mathrm{C}_{*}\left(W_{1}\right)$ and $\gamma_{2} \in \mathrm{C}_{*}\left(W_{2}\right),\left\langle\omega, \gamma_{1} \times \gamma_{2}\right\rangle=0$, then $\omega=0$.

Proof The proof is analogous to the proof of the injectivity of the map (4) in Lemma 5.1.

\subsection{Minimal forms}

In this section we give the first version of "semi-algebraic differential forms" following [19, Section 8.3].

Let $X \subset \mathbb{R}^{N}$ be a semi-algebraic set and let $f_{0}, f_{1}, \ldots, f_{k}: X \rightarrow \mathbb{R}$ be semi-algebraic functions. We will define a cochain

$$
\lambda\left(f_{0} ; f_{1}, \ldots, f_{k}\right) \in \mathrm{C}^{k}(X)
$$

which, when $X$ and the $f_{i}$ 's are smooth, is just the smooth cochain $f_{0} d f_{1} \wedge \cdots \wedge d f_{k} \in$ $\Omega_{\mathcal{C}^{\infty}}^{k}(X)$. To define $\lambda\left(f_{0} ; f_{1}, \ldots, f_{k}\right)$ in the general case, set

$$
f=\left(f_{0}, f_{1}, \ldots, f_{k}\right): X \longrightarrow \mathbb{R}^{k+1}
$$

which is a semi-algebraic map. Recall that an element of $\mathrm{C}_{k}\left(\mathbb{R}^{k+1}\right)$ is a $k$-current in $\mathbb{R}^{k+1}$, and hence can be evaluated on smooth $k$-forms with compact support in $\mathbb{R}^{k+1}$. For a semi-algebraic $k$-chain $\gamma \in \mathrm{C}_{k}(X)$, define

$$
\left\langle\lambda\left(f_{0} ; f_{1}, \ldots, f_{k}\right), \gamma\right\rangle=f_{*}(\gamma)\left(\rho \cdot x_{0} d x_{1} \wedge \cdots \wedge d x_{k}\right),
$$

where $x_{0}, \ldots, x_{k}$ are the coordinates in $\mathbb{R}^{k+1}$ and $\rho: \mathbb{R}^{k+1} \rightarrow \mathbb{R}$ is a smooth bump function with compact support that takes the value 1 on $\operatorname{spt}\left(f_{*}(\gamma)\right)$. Clearly the result is independent of the choice of $\rho$, and abusing notation we will simply write $f_{*}(\gamma)\left(x_{0} d x_{1} \wedge \cdots \wedge d x_{k}\right)$.

Definition 5.4 We denote by $\Omega_{\min }^{k}(X)$ the subgroup of $\mathrm{C}^{k}(X)$ generated by the cochains $\lambda\left(f_{0} ; f_{1}, \ldots, f_{k}\right)$. Its elements are called the minimal forms.

The pullback of a minimal form along a semi-algebraic map is again a minimal form. More precisely, let $g: X \rightarrow Y$ be a semi-algebraic map and let $f_{i}: Y \rightarrow \mathbb{R}$ be semialgebraic functions for $0 \leq i \leq k$. It is immediate to check that

$$
g^{*}\left(\lambda\left(f_{0} ; f_{1}, \ldots, f_{k}\right)\right)=\lambda\left(f_{0} \circ g ; f_{1} \circ g, \ldots, f_{k} \circ g\right) .
$$

The following formula implies the coboundary of a minimal form is also a minimal form. 
Proposition $5.5 \delta\left(\lambda\left(f_{0} ; f_{1}, \ldots, f_{k}\right)\right)=\lambda\left(1 ; f_{0}, f_{1}, \ldots, f_{k}\right)$.

Proof Let $\gamma \in \mathrm{C}_{k+1}(X)$. Recall that the boundary $\partial$ on $\mathrm{C}_{k+1}(X) \subset \mathcal{D}_{k+1}(X)$ is defined as the adjoint of $d$ on $\mathcal{D}^{k}\left(\mathbb{R}^{N}\right)$. Thus using the definitions and Corollary 3.7, we have

$$
\begin{aligned}
\left\langle\delta\left(\lambda\left(f_{0} ; f_{1}, \ldots, f_{k}\right), \gamma\right\rangle\right. & =\left\langle\lambda\left(f_{0} ; f_{1}, \ldots, f_{k}, \partial \gamma\right\rangle\right. \\
& =\left(\left(f_{0}, f_{1}, \ldots, f_{k}\right)_{*}(\partial \gamma)\right)\left(x_{0} d x_{1} \cdots d x_{k}\right) \\
& =\left(\partial\left(\left(f_{0}, f_{1}, \ldots, f_{k}\right)_{*}(\gamma)\right)\right)\left(x_{0} d x_{1} \cdots d x_{k}\right) \\
& =\left(\left(f_{0}, f_{1}, \ldots, f_{k}\right)_{*}(\gamma)\right)\left(d x_{0} d x_{1} \cdots d x_{k}\right) \\
& =\left(\left(1, f_{0}, f_{1}, \ldots, f_{k}\right)_{*}(\gamma)\right)\left(t d x_{0} d x_{1} \cdots d x_{k}\right) \\
& =\left\langle\lambda\left(1 ; f_{0}, f_{1}, \ldots, f_{k}\right), \gamma\right\rangle .
\end{aligned}
$$

The above implies that $\Omega_{\min }^{*}(X)$ is a cochain complex. We will later define an algebra structure on it using a certain cross product. However, in order to prove that this product is well defined, we first need to show in the three following propositions that minimal forms are well approximated by smooth forms.

First we have continuity of $\langle\mu,-\rangle$.

Proposition 5.6 Let $X$ be a semi-algebraic set and let $\mu \in \Omega_{\min }^{k}(X)$. If $\gamma_{n} \stackrel{\text { SA }}{\rightarrow} \gamma$ in $\mathrm{C}_{k}(X)$, then $\lim _{n \rightarrow \infty}\left\langle\mu, \gamma_{n}\right\rangle=\langle\mu, \gamma\rangle$.

Proof By linearity, it is enough to prove this when $\mu=\lambda\left(f_{0} ; f_{1}, \ldots, f_{k}\right)$ for some semi-algebraic functions $f_{j}: X \rightarrow \mathbb{R}$. Set $f=\left(f_{0}, f_{1}, \ldots, f_{k}\right): X \rightarrow \mathbb{R}^{k+1}$. By Proposition 4.2 and Proposition 4.4, $f_{*}\left(\gamma_{n}\right) \rightarrow f_{*}(\gamma)$, and so

$$
\begin{aligned}
\lim _{n \rightarrow \infty}\left\langle\mu, \gamma_{n}\right\rangle & =\lim _{n \rightarrow \infty}\left(f_{*}\left(\gamma_{n}\right)\left(x_{0} d x_{1} \ldots d x_{k}\right)\right) \\
& =\left(f_{*}(\gamma)\left(x_{0} d x_{1} \ldots d x_{k}\right)\right) \\
& =\langle\mu, \gamma\rangle .
\end{aligned}
$$

Notice that we have implicitly used the fact that the bump function $\rho$ in front of $x_{0} d x_{1} \ldots d x_{k}$ can be chosen to be the same for all $\gamma_{n}$ because, by the SA convergence, $\bigcup_{n=1}^{\infty} \operatorname{spt}\left(\gamma_{n}\right)$ is included in some compact set.

We next show that a minimal form is smooth at a "generic point" and that any minimal form is determined by its values at such points.

Proposition 5.7 Let $X$ be a semi-algebraic set and let $\mu \in \Omega_{\min }^{k}(X)$. There exists a semi-algebraic smooth submanifold $W$ that is open and dense in $X$ and such that $\left.\mu\right|_{W}$ is smooth. 
Proof Suppose $\mu=\sum_{i=1}^{p} \lambda\left(f_{0}^{(i)} ; f_{1}^{(i)}, \ldots, f_{k}^{(i)}\right)$ for some semi-algebraic functions $f_{j}^{(i)}: X \rightarrow \mathbb{R}$. Take a stratification of $X$ into smooth submanifolds on which the $f_{j}^{(i)}$ are smooth. Let $W$ be the union of the maximal strata. This is open and dense in $X$ and $\left.\mu\right|_{W}=\sum_{i} f_{0}^{(i)} d f_{1}^{(i)} \ldots d f_{k}^{(i)}$ is a smooth form.

Proposition 5.8 Let $X$ be a semi-algebraic set and let $\mu \in \Omega_{\min }^{k}(X)$. If $X_{0} \subset X$ is a dense semi-algebraic subset and if $\left.\mu\right|_{X_{0}}=0$ then $\mu=0$.

Proof Let $\gamma \in \mathrm{C}_{k}(X)$. Since $X_{0}$ is dense in $X$, by Proposition 4.3 there exists a sequence $\left(\gamma_{n}\right)_{n \geq 1}$ in $\mathrm{C}_{k}\left(X_{0}\right)$ converging semi-algebraically to $\gamma$. By hypothesis, $\left\langle\mu, \gamma_{n}\right\rangle=0$, and we conclude by Proposition 5.6 that $\mu=0$ since it is 0 when evaluated at an arbitrary chain $\gamma$.

Proposition 5.9 Let $X_{1}$ and $X_{2}$ be semi-algebraic sets and let $\mu \in \Omega_{\min }^{k}\left(X_{1} \times X_{2}\right)$. If $\left\langle\mu, \gamma_{1} \times \gamma_{2}\right\rangle=0$ for all $\gamma_{1} \in \mathrm{C}_{*}\left(X_{1}\right)$ and $\gamma_{2} \in \mathrm{C}_{*}\left(X_{2}\right)$ then $\mu=0$.

Proof By Proposition 5.7 there exists a semi-algebraic smooth submanifold $W$, open and dense in $X_{1} \times X_{2}$, such that $\omega:=\left.\mu\right|_{W}$ is smooth. We show that $\omega=0$. Let $\left(x_{1}, x_{2}\right) \in W$. There exist smooth neighborhoods $U_{i} \subset X_{i}$ of $x_{i}$ for $i=1,2$ such that $U_{1} \times U_{2} \subset W$. By Lemma 5.3, $\left.\omega\right|_{U_{1} \times U_{2}}=0$. This implies that $\omega=0$. Since $W$ is dense in $X$, we deduce the desired result by Proposition 5.8.

Proposition 5.10 Let $X_{1}$ and $X_{2}$ be semi-algebraic sets. There is a degree-preserving linear map

$$
\times: \Omega_{\min }^{*}\left(X_{1}\right) \otimes \Omega_{\min }^{*}\left(X_{2}\right) \longrightarrow \Omega_{\min }^{*}\left(X_{1} \times X_{2}\right)
$$

given by

$$
\left\langle\mu_{1} \times \mu_{2}, \gamma_{1} \times \gamma_{2}\right\rangle=\left\langle\mu_{1}, \gamma_{1}\right\rangle \cdot\left\langle\mu_{2}, \gamma_{2}\right\rangle
$$

for $\mu_{i} \in \Omega_{\min }^{*}\left(X_{i}\right)$ and $\gamma_{i} \in \mathrm{C}_{*}\left(X_{i}\right)$. This formula satisfies the Leibniz rule

$$
\delta\left(\mu_{1} \times \mu_{2}\right)=\delta\left(\mu_{1}\right) \times \mu_{2}+(-1)^{\operatorname{deg}\left(\mu_{1}\right)} \mu_{1} \times \delta\left(\mu_{2}\right) .
$$

Further, let $T: X_{1} \times X_{2} \rightarrow X_{2} \times X_{1}$ be the twisting map, given by $\left(x_{1}, x_{2}\right) \mapsto\left(x_{2}, x_{1}\right)$. Then

$$
T^{*}\left(\mu_{2} \times \mu_{1}\right)=(-1)^{\operatorname{deg}\left(\mu_{1}\right) \operatorname{deg}\left(\mu_{2}\right)} \mu_{1} \times \mu_{2} .
$$

Proof Let $k_{i}$ be nonnegative integers, let $f_{0}^{(i)}, \ldots, f_{k_{i}}^{(i)}: X_{i} \rightarrow \mathbb{R}$ be semi-algebraic functions for $i=1,2$, and set $\mu_{i}=\lambda\left(f_{0}^{(i)} ; f_{1}^{(i)}, \ldots, f_{k_{i}}^{(i)}\right) \in \Omega_{\min }^{k_{i}}\left(X_{i}\right)$. Consider also the projections $\operatorname{pr}_{i}: X_{1} \times X_{2} \rightarrow X_{i}$. Set

(6) $\mu_{1} \times \mu_{2}=\lambda\left(\left(f_{0}^{(1)} \mathrm{pr}_{1}\right) \cdot\left(f_{0}^{(2)} \mathrm{pr}_{2}\right) ; f_{1}^{(1)} \mathrm{pr}_{1}, \ldots, f_{k_{1}}^{(1)} \mathrm{pr}_{1}, f_{1}^{(2)} \mathrm{pr}_{2}, \ldots, f_{k_{2}}^{(2)} \mathrm{pr}_{2}\right)$ 
and extend bilinearly. It is straightforward to check that this definition satisfies Equation (5) of the proposition.

The fact that the minimal form $\mu_{1} \times \mu_{2}$ is characterized by Equation (5), and in particular that the right side of (6) is independent of the choice of the representatives of the $\mu_{i}$ 's, is a consequence of Proposition 5.9.

The Leibniz and twisting formulas are consequences of the corresponding formulas for chains in Proposition 3.8.

For $\mu_{1}, \mu_{2} \in \Omega_{\min }^{*}(X)$, we define a multiplication on $\Omega_{\min }^{*}(X)$ by

$$
\mu_{1} \cdot \mu_{2}=\Delta^{*}\left(\mu_{1} \times \mu_{2}\right)
$$

where $\Delta: X \rightarrow X \times X$ is the diagonal map. It is immediate from the previous proposition that this multiplication satisfies the Leibniz formula and is graded commutative.

In conclusion we have:

Theorem 5.11 The above construction of minimal forms defines a contravariant functor $\Omega_{\min }^{*}:$ SemiAlg $\rightarrow$ CDGA.

Remark 5.12 Note that we do not have the analog of de Rham theorem for the functor $\Omega_{\min }^{*}$; in general $H\left(\Omega_{\min }^{*}(X)\right)$ is not isomorphic to $H^{*}(X ; \mathbb{R})$. For example, consider the contractible semi-algebraic set $X=[1,2]$ and the minimal 1 -form $\mu=\lambda\left(f_{0} ; f_{1}\right)$ with $f_{0}, f_{1}: X \rightarrow \mathbb{R}$ defined by $f_{0}(t)=1 / t$ and $f_{1}(t)=t$ for $t \in[1,2]$. In other words, $\mu=d t / t$, and this is a smooth form which is a $\delta$-cocycle but it is not a $\delta$-coboundary in $\Omega_{\min }^{*}(X)$ because the map $t \mapsto \log (t)$ is not semi-algebraic. This is an issue we will get around by enlarging the cochain complex $\Omega_{\min }^{*}$ in Section 5.4.

\subsection{Strongly continuous families of chains}

Definition 5.13 Let $f: Y \rightarrow X$ be a semi-algebraic map. A strongly continuous family of chains or, shortly, a (strongly) continuous chain of dimension $l$ over $X$ along $f$ is a map

$$
\Phi: X \longrightarrow \mathrm{C}_{l}(Y)
$$

such that there exist:

(1) a finite semi-algebraic stratification $\left\{S_{\alpha}\right\}_{\alpha \in I}$ of $X$, and, for each $\alpha \in I$,

(2) an oriented compact semi-algebraic manifold $F_{\alpha}$ of dimension $l$;

(3) a semi algebraic map $g_{\alpha}: \overline{S_{\alpha}} \times F_{\alpha} \rightarrow Y$, where $\overline{S_{\alpha}}$ is the closure of $S_{\alpha}$ in $X$, satisfying: 
(a) the diagram

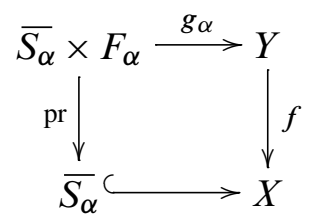

commutes;

(b) for each $\alpha \in I$ and $x \in \overline{S_{\alpha}}, \Phi(x)=g_{\alpha *}\left(\llbracket\{x\} \times F_{\alpha} \rrbracket\right)$.

We say that the family $\left\{\left(S_{\alpha}, F_{\alpha}, g_{\alpha}\right)\right\}_{\alpha \in I}$ trivializes or represents the continuous chain $\Phi$ and we denote by $C_{l}^{\text {str }}(f: Y \rightarrow X)$ the set of strongly continuous $l$-chains.

Remark 5.14 Note that if we had asked that the maps $g_{\alpha}$ be defined only on $S_{\alpha} \times F_{\alpha}$ instead of on the closure $\overline{S_{\alpha}} \times F_{\alpha}$ in the above definition, we would obtain a sort of semi-algebraic parametrized chain in $Y$ over $X$, lacking a continuity condition. We would therefore not have the Leibniz formula from Proposition 5.17. It appears that Kontsevich and Soibelman had something weaker in mind in their definition of continuous chains [19, Definition 22] and we have thus added strongly and the adornment str in order to distinguish our definition from theirs. See the discussion in Section 9.

It is clear that if $\left\{S_{\beta}^{\prime}\right\}_{\beta \in J}$ is a stratification of $X$ refining the stratification $\left\{S_{\alpha}\right\}_{\alpha \in I}$ of the above definition, that is, if for each $\beta \in J$ there exists $\alpha=\alpha(\beta) \in I$ such that $S_{\beta}^{\prime} \subset S_{\alpha}$, then there is an induced trivialization $\left\{\left(S_{\beta}^{\prime}, F_{\beta}, g_{\beta}\right)\right\}_{\beta \in J}$ with $F_{\beta}=F_{\alpha(\beta)}$ and $g_{\beta}=g_{\alpha(\beta)} \mid \overline{S_{\beta}^{\prime}} \times F_{\beta}$.

Consider the set

$$
\operatorname{map}\left(X, \mathrm{C}_{l}(Y)\right):=\left\{\Phi: X \rightarrow \mathrm{C}_{l}(Y)\right\}
$$

of all maps of sets from $X$ to the $l$-chains on $Y$. This set has an abelian group structure induced by that on $\mathrm{C}_{l}(Y)$. Moreover, if $\Phi \in \operatorname{map}\left(X, \mathrm{C}_{l}(Y)\right)$ we define its boundary $\partial \Phi \in \operatorname{map}\left(X, \mathrm{C}_{l-1}(Y)\right)$ by the formula

$$
(\partial \Phi)(x)=\partial(\Phi(x)), x \in X .
$$

Let $\operatorname{map}\left(X, \mathrm{C}_{*}(Y)\right)=\bigoplus_{l \geq 0} \operatorname{map}\left(X, \mathrm{C}_{l}(Y)\right)$ and $\mathrm{C}_{*}^{\mathrm{str}}(Y \rightarrow X)=\bigoplus_{l \geq 0} \mathrm{C}_{l}^{\mathrm{str}}(Y \rightarrow X)$. It is clear that the former is a chain complex of abelian groups and that the latter is a subset.

Lemma 5.15 $\mathrm{C}_{*}^{\mathrm{str}}(Y \rightarrow X)$ is a chain subcomplex of map $\left(X, \mathrm{C}_{*}(Y)\right)$.

Proof We prove first that each $\mathrm{C}_{l}^{\mathrm{str}}(Y \rightarrow X)$ is a subgroup. 
The zero element of $\operatorname{map}\left(X, \mathrm{C}_{l}(Y)\right)$ is an element of $\mathrm{C}_{l}^{\mathrm{str}}(Y \rightarrow X)$ since it is represented by $\{(X, \varnothing, X \times \varnothing=\varnothing \hookrightarrow Y)\}$.

Let $\Phi, \Phi^{\prime} \in \mathrm{C}_{l}^{\mathrm{str}}(Y \rightarrow X)$. By taking a common refinement we can suppose that these continuous chains are represented by $\left\{\left(S_{\alpha}, F_{\alpha}, g_{\alpha}\right)\right\}_{\alpha \in I}$ and $\left\{\left(S_{\alpha}, F_{\alpha}^{\prime}, g_{\alpha}^{\prime}\right)\right\}_{\alpha \in I}$ respectively. Letting $g_{\alpha}^{\prime \prime}=\left(g_{\alpha}, g_{\alpha}^{\prime}\right): \overline{S_{\alpha}} \times\left(F_{\alpha} \sqcup F_{\alpha}^{\prime}\right) \rightarrow Y$, it is clear that $\Phi+\Phi^{\prime}$ is represented by $\left\{\left(S_{\alpha}, F_{\alpha} \sqcup F_{\alpha}^{\prime}, g_{\alpha}^{\prime \prime}\right)\right\}_{\alpha \in I}$, so it is also a continuous chain.

The inverse of a continuous chain can be represented by reversing the orientations of the manifolds $F_{\alpha}$.

It remains to prove that $\partial\left(\mathrm{C}_{l}^{\mathrm{str}}(Y \rightarrow X)\right) \subset \mathrm{C}_{l-1}^{\mathrm{str}}(Y \rightarrow X)$. Indeed suppose that $\Phi \in \mathrm{C}_{l}^{\mathrm{str}}(Y \rightarrow X)$ is represented by $\left\{\left(S_{\alpha}, F_{\alpha}, g_{\alpha}\right)\right\}_{\alpha \in I}$. Then by Theorem 3.5, $\partial \Phi$ is represented by $\left\{\left(S_{\alpha}, \partial F_{\alpha}, \partial g_{\alpha}\right)\right\}_{\alpha \in I}$ where $\partial g_{\alpha}$ is the restriction of $g_{\alpha}$ to $\overline{S_{\alpha}} \times \partial F_{\alpha}$.

Let $f: Y \rightarrow X$ be a semi-algebraic map, let $\gamma \in \mathrm{C}_{k}(X)$ and let $\Phi \in \mathrm{C}_{l}^{\mathrm{str}}(Y \rightarrow X)$. We construct a chain $\gamma \ltimes \Phi \in \mathrm{C}_{k+l}(Y)$ as follows. Take a trivialization $\left\{\left(S_{\alpha}, F_{\alpha}, g_{\alpha}\right)\right\}$ of $\Phi$. We can suppose that the stratification is fine enough to be adapted to $\gamma$ in the sense that there exist integers $n_{\alpha}$ such that

$$
\gamma=\sum_{\alpha} n_{\alpha} \cdot \llbracket \overline{S_{\alpha}} \rrbracket
$$

where $\overline{S_{\alpha}}$ are compact oriented semi-algebraic manifolds (take for example a stratification whose restriction to $\operatorname{spt}(\gamma)$ is a suitable triangulation.) Set

$$
\gamma \ltimes \Phi=\sum_{\alpha} n_{\alpha} \cdot g_{\alpha *}\left(\llbracket \overline{S_{\alpha}} \times F_{\alpha} \rrbracket\right) .
$$

We will prove in Proposition 5.17 that this operation is well-defined and satisfies the Leibniz formula. In order to do so we need the following version of Fubini's Theorem.

Lemma 5.16 Let $g: S \times F \rightarrow Y$ and $g^{\prime}: S \times F^{\prime} \rightarrow Y$ be two semi-algebraic maps such that $S, F$, and $F^{\prime}$ are compact oriented semi-algebraic manifolds with $\operatorname{dim} F=\operatorname{dim} F^{\prime}$. Suppose that for all $x \in S$ we have $g_{*}(\llbracket\{x\} \times F \rrbracket)=g_{*}^{\prime}\left(\llbracket\{x\} \times F^{\prime} \rrbracket\right)$ in $\mathrm{C}_{*}(Y)$. Then $g_{*}(\llbracket S \times F \rrbracket)=g_{*}^{\prime}\left(\llbracket S \times F^{\prime} \rrbracket\right)$.

Proof Set $T=F \sqcup-F^{\prime}$ where $-F^{\prime}$ is $F^{\prime}$ with the opposite orientation and consider the map $f=g \sqcup g^{\prime}: S \times T \rightarrow Y$. By linearity, for each $x \in S$ we have $f_{*}(\llbracket\{x\} \times T \rrbracket)=0$ and what we now have to show is that $f_{*}(\llbracket S \times T \rrbracket)=0$.

Set $n=\operatorname{dim}(S)+\operatorname{dim}(T)$. Without loss of generality we can assume that $Y=f(S \times T)$, and hence $\operatorname{dim}(Y) \leq n$. If $\operatorname{dim}(Y)<n$ then the conclusion of the lemma is immediate, so we assume $\operatorname{dim}(Y)=n$. 
Let $W$ be a semi-algebraic smooth submanifold that is open and dense in $S \times T$ and let $Z \subset S \times T$ be the closure of

$$
\left(\begin{array}{l|l}
S \times T \backslash W) \cup\{(s, t) \in W & \begin{array}{l}
f \text { is not smooth at }(s, t) \text { or it is smooth } \\
\text { but } d f(s, t) \text { is of rank }<n
\end{array}
\end{array}\right\}
$$

Then $\operatorname{dim} f(Z)<n$ and there exists a nonempty smooth $n$-dimensional semi-algebraic submanifold $Y_{0} \subset Y \backslash f(Z)$ such that $\operatorname{spt}\left(f_{*}(\llbracket S \times T \rrbracket)\right) \subset \overline{Y_{0}}$. Set $X_{0}=f^{-1}\left(Y_{0}\right)$ and let $f_{0}$ be the restriction of $f$ to $X_{0}$. Then $f_{0}$ is locally a diffeomorphism onto its image and for $y_{0} \in Y$ we have $f^{-1}\left(y_{0}\right)=f_{0}^{-1}\left(y_{0}\right)$. This set is discrete, and hence finite by compactness of $S \times T$.

The multiplicity in $f_{*}(\llbracket S \times T \rrbracket)$ of the neighborhood of a point $y_{0} \in Y_{0}$ is given by the formula

$$
\operatorname{mult}\left(y_{0}\right)=\sum_{x \in f^{-1}\left(y_{0}\right)} \operatorname{sgn}(\operatorname{det}(d f(x))) .
$$

We have

$$
f^{-1}\left(y_{0}\right)=\left\{\left(s_{1}, t_{1}^{1}\right), \ldots,\left(s_{1}, t_{1}^{r_{1}}\right), \ldots,\left(s_{p}, t_{p}^{1}\right), \ldots,\left(s_{p}, t_{p}^{r_{p}}\right)\right\}
$$

with the $s_{i}$ are all distinct in $S$, and so the multiplicity can be rewritten as

$$
\sum_{i=1}^{p}\left(\sum_{j=1}^{r_{i}} \operatorname{sgn}\left(\operatorname{det}\left(d f\left(s_{i}, t_{i}^{j}\right)\right)\right)\right) .
$$

If this expression is nonzero then one of the terms in the brackets has to be nonzero, but this contradicts the fact that $f_{*}\left(\llbracket\left\{s_{i}\right\} \times T \rrbracket\right)=0$. So the multiplicity at $y_{0}$ is zero.

Proposition 5.17 The formula (7) above defines a natural linear map

$$
\begin{aligned}
\ltimes: \mathrm{C}_{k}(X) \otimes \mathrm{C}_{l}^{\mathrm{str}}(Y & \rightarrow X) \longrightarrow \mathrm{C}_{k+l}(Y) \\
\gamma \otimes \Phi & \longrightarrow \gamma \ltimes \Phi
\end{aligned}
$$

which satisfies the Leibniz formula $\partial(\gamma \ltimes \Phi)=(\partial \gamma) \ltimes \Phi+(-1)^{\operatorname{deg}(\gamma)} \gamma \ltimes(\partial \Phi)$.

Proof We need to prove that the right side of (7) is independent of the choice of trivialization of $\Phi$. First, it is clear that if we take a refinement of the stratification and consider the induced trivialization, the right side is unchanged. Therefore it is enough to prove the invariance for two trivializations $\left\{\left(S_{\alpha}, F_{\alpha}, g_{\alpha}\right)\right\}$ and $\left\{\left(S_{\alpha}, F_{\alpha}^{\prime}, g_{\alpha}^{\prime}\right)\right\}$ with the same underlying stratification. But this is an immediate consequence of Lemma 5.16.

By linearity of $\ltimes$ and $\partial$, it is enough to check the Leibniz formula when $\gamma=\llbracket S \rrbracket$, with $S$ a closed subset of $X$ that is a compact oriented manifold over which $\Phi$ is 
trivial in the sense that there is a semi-algebraic map $g: S \times F \rightarrow Y$ with $F$ a compact oriented manifold and, for each $x \in S, \Phi(x)=g_{*}(\llbracket\{x\} \times F \rrbracket)$. Using Theorem 3.5 we then get

$$
\begin{aligned}
\partial(\gamma \ltimes \Phi) & =\partial\left(g_{*}(\llbracket S \times F \rrbracket)\right) \\
& \left.=g_{*}(\llbracket(\partial S) \times F \rrbracket)+(-1)^{\operatorname{dim}(S)} g_{*}(\llbracket S \times(\partial F) \rrbracket)\right) \\
& =(\partial \gamma) \ltimes \Phi+(-1)^{\operatorname{deg}(\gamma)} \gamma \ltimes(\partial \Phi) .
\end{aligned}
$$

We now define the pullback of continuous chains.

Proposition 5.18 Suppose given a pullback of semi-algebraic sets

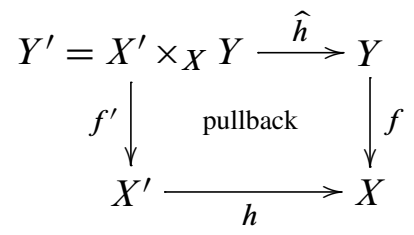

and $\Phi \in \mathrm{C}_{l}^{\mathrm{str}}(Y \rightarrow X)$. There exists a unique continuous chain $\Phi^{\prime} \in \mathrm{C}_{l}^{\mathrm{str}}\left(Y^{\prime} \rightarrow X^{\prime}\right)$ such that, for each $x^{\prime} \in X^{\prime}, \widehat{h}_{*}\left(\Phi^{\prime}\left(x^{\prime}\right)\right)=\Phi\left(h\left(x^{\prime}\right)\right)$.

Proof Set $x=h\left(x^{\prime}\right)$. Since $Y^{\prime}$ is the pullback, the restriction of $\hat{h}$ to fibers over $x^{\prime}$ and $x$ induces an isomorphism $\hat{h}_{x^{\prime}}: f^{\prime-1}\left(x^{\prime}\right) \cong f^{\prime-1}(x)$. Since the support of $\Phi(x)$ is included in the fiber over $x$ we deduce that the value $\Phi^{\prime}\left(x^{\prime}\right)$ is completely determined as $\left(h_{x^{\prime}}^{-1}\right)_{*}(\Phi(x))$. This implies the uniqueness.

The fact that $\Phi^{\prime}$ is indeed a continuous chain comes from existence of a trivialization obtained by taking the pullback of a trivialization of $\Phi$ in a straightforward way.

Definition 5.19 We call the continuous chain $\Phi^{\prime}$ from the previous proposition the pullback of $\Phi$ along $h$ and we denote it by $h^{*}(\Phi)$. In case of an inclusion $h: X^{\prime} \hookrightarrow X$, we also write $\left.\Phi\right|_{X^{\prime}}=h^{*}(\Phi)$.

It is easy to check that the pullback operation

$$
h^{*}: \mathrm{C}_{*}^{\mathrm{str}}(Y \rightarrow X) \longrightarrow \mathrm{C}_{*}^{\mathrm{str}}\left(Y^{\prime} \rightarrow X^{\prime}\right)
$$

is a morphism of chain complexes. Also, given $\Phi_{1} \in \mathrm{C}_{l_{1}}^{\mathrm{str}}\left(Y_{1} \rightarrow X_{1}\right)$ and $\Phi_{2} \in$ $\mathrm{C}_{l_{2}}^{\mathrm{str}}\left(Y_{2} \rightarrow X_{2}\right)$, we can construct in the obvious way a continuous chain $\Phi_{1} \times \Phi_{2} \in$ $\mathrm{C}_{l_{1}+l_{2}}^{\mathrm{str}}\left(Y_{1} \times Y_{2} \rightarrow X_{1} \times X_{2}\right)$ characterized by

$$
\left(\Phi_{1} \times \Phi_{2}\right)\left(x_{1}, x_{2}\right)=\Phi_{1}\left(x_{1}\right) \times \Phi_{2}\left(x_{2}\right) .
$$


The Leibniz formula

$$
\partial\left(\Phi_{1} \times \Phi_{2}\right)=\partial\left(\Phi_{1}\right) \times \Phi_{2}+(-1)^{\operatorname{deg}\left(\Phi_{1}\right)} \Phi_{1} \times \partial\left(\Phi_{2}\right)
$$

clearly holds.

Now let $F$ be a compact oriented semi-algebraic manifold of dimension $l$ and let $X$ be a semi-algebraic set. We define the constant continuous chain $\widehat{F} \in \mathrm{C}^{\mathrm{str}}\left(\mathrm{pr}_{1}: X \times F \rightarrow X\right)$ by $\hat{F}(x)=\llbracket\{x\} \times F \rrbracket$.

\subsection{The cochain complex of PA forms}

As we explained in Remark 5.12, $\Omega_{\min }^{*}\left(\Delta^{k}\right)$ is not acyclic. In this section we will enlarge the cochain complex $\Omega_{\min }^{*}(X)$ into a cochain complex $\Omega_{\mathrm{PA}}^{*}(X)$ which will satisfy the Poincaré lemma.

Let $f: Y \rightarrow X$ be a semi-algebraic map, let $\Phi \in \mathrm{C}_{l}^{\text {str }}(f: Y \rightarrow X)$ be a continuous $l$-chain and let $\mu \in \Omega_{\min }^{k+l}(Y)$. We define a cochain $f_{\Phi} \mu \in \mathrm{C}^{k}(X)$ by

$$
\left\langle f_{\Phi} \mu, \gamma\right\rangle=\langle\mu, \gamma \ltimes \Phi\rangle \text { for } \gamma \in \mathrm{C}_{k}(X) \text {. }
$$

Definition 5.20 A PA form of degree $k$ in $X$ is a cochain in $X$ of the form $f_{\Phi} \mu$ as defined in Equation (8) for some semi-algebraic map $f: Y \rightarrow X$, some continuous chain $\Phi \in \mathrm{C}_{l}^{\mathrm{str}}(f: Y \rightarrow X)$, and some minimal form $\mu \in \Omega_{\min }^{k+l}(Y)$. We denote by $\Omega_{\mathrm{PA}}^{k}(X) \subset \mathrm{C}^{k}(X)$ the subset of all PA forms in $X$.

Definition 5.21 Given a closed semi-algebraic subset $A \subset X$, we say that a PA form $\alpha \in \Omega_{\mathrm{PA}}^{k}(X)$ is a trivial fiber integral over $A$ if there exists a continuous chain $\Phi \in \mathrm{C}_{l}^{\mathrm{str}}(Y \rightarrow X)$ and a minimal form $\mu \in \Omega_{\min }^{k+l}$ such that $\alpha=f_{\Phi} \mu$ and $A$ is the relative closure of a stratum of some trivialization of $\Phi$.

Notice that by definition of a PA form there always exists a (semi-open) triangulation of the space such that the PA form is a trivial fiber integral over the relative closure of each simplex. When $\alpha \in \Omega_{\mathrm{PA}}^{k}(X)$ is a trivial fiber integral over $A$ then by definition $\left.\alpha\right|_{A}=f_{\widehat{F}} \mu$ for some compact oriented semi-algebraic manifold $F$ and some minimal form $\mu \in \Omega_{\min }^{k+\operatorname{dim}(F)}(A \times F)$, where $\widehat{F} \in \mathrm{C}_{\operatorname{dim}(F)}^{\mathrm{str}}(\operatorname{pr}: A \times F \rightarrow A)$ is the constant continuous chain introduced before Definition 8.1.

Proposition 5.22 $\Omega_{\mathrm{PA}}^{k}(X)$ is a subgroup of $\mathrm{C}^{k}(X)$.

Proof Let $\alpha_{i}=f_{\Phi_{i}} \mu_{i}$ be two PA forms in $\Omega_{\mathrm{PA}}^{k}(X)$ with $\Phi_{i} \in \mathrm{C}_{l_{i}}^{\mathrm{str}}\left(f_{i}: Y_{i} \rightarrow X\right)$ and $\mu_{i} \in \Omega_{\min }^{k+l_{i}}\left(Y_{i}\right)$, for $i=1,2$. 
We first show that we can assume that $l_{1}=l_{2}$. For concreteness, suppose that $l_{1} \leq l_{2}$ and set $r=l_{2}-l_{1} \geq 0$. There is a natural isomorphism $\mathrm{C}_{r}^{\mathrm{str}}\left([0,1]^{r} \rightarrow *\right) \cong \mathrm{C}_{r}\left([0,1]^{r}\right)$ given by $\Phi \mapsto \Phi(*)$, and hence we can realize $\llbracket[0,1]^{r} \rrbracket$ as an element of $\mathrm{C}_{r}^{\mathrm{str}}\left([0,1]^{r} \rightarrow *\right)$. Consider also the smooth minimal form $d t_{1} \cdots d t_{r}=\lambda\left(1 ; t_{1}, \ldots, t_{n}\right) \in \Omega_{\min }^{r}\left([0,1]^{r}\right)$ where $t_{1}, \ldots, t_{r}$ are the coordinates in $[0,1]^{r}$. Then

$$
\alpha_{1}=f_{\Phi_{1} \times \llbracket[0,1]^{r} \rrbracket} \mu_{1} \times d t_{1} \cdots d t_{r},
$$

where $\Phi_{1} \times \llbracket[0,1]^{r} \rrbracket \in \mathrm{C}_{l_{2}}^{\mathrm{str}}\left(Y_{1} \times[0,1]^{r} \rightarrow X \times *\right)$ so we can assume that $l_{1}=l_{2}$.

Now consider the obvious continuous chain $\Phi_{1}+\Phi_{2} \in \mathrm{C}_{l_{1}}^{\mathrm{str}}\left(Y_{1} \sqcup Y_{2} \rightarrow X\right)$ and the minimal form $\mu_{1}+\mu_{2} \in \Omega_{\min }^{k+l_{1}}\left(Y_{1} \sqcup Y_{2}\right)$. Then

$$
f_{\Phi_{1}} \mu_{1}+f_{\Phi_{2}} \mu_{2}=\int_{\Phi_{1}+\Phi_{2}} \mu_{1}+\mu_{2},
$$

and so the sum of two PA forms is still a PA form. It is obvious that 0 is a PA form as well as is the inverse of a PA form.

The coboundary of a PA form is again a PA form because of the following.

Lemma $5.23 \delta\left(f_{\Phi} \mu\right)=f_{\Phi} \delta \mu+(-1)^{\operatorname{deg}(\mu)-\operatorname{deg}(\Phi)} f_{\partial \Phi} \mu$.

Proof Evaluate both side on a chain $\gamma$ using the Leibniz formula for the boundary of $\gamma \ltimes \Phi$ and unravelling definitions.

It is also clear that $\Omega_{\min }^{k}(X) \subset \Omega_{\mathrm{PA}}^{k}(X)$ because a minimal form $\mu$ on $X$ can be written as $f_{\hat{*}} \mu$ where $\hat{*} \in \mathrm{C}_{0}^{\mathrm{str}}(X \stackrel{\equiv}{\rightarrow} X)$ is the constant continuous 0 -chain defined by $\widehat{*}(x)=\llbracket\{x\} \rrbracket$.

If $g: X^{\prime} \rightarrow X$ is a semi-algebraic map then $g^{*}\left(\Omega_{\mathrm{PA}}^{*}(X)\right) \subset \Omega_{\mathrm{PA}}^{*}\left(X^{\prime}\right)$ because of the formula $g^{*}\left(f_{\Phi} \mu\right)=\int_{g^{*} \Phi} g^{*} \mu$, which is straightforward to check.

The following is a direct consequence of Proposition 3.3.

Proposition 5.24 Let $\alpha \in \Omega_{\mathrm{PA}}(X)$. If $\operatorname{deg}(\alpha)>\operatorname{dim}(X)$ then $\alpha=0$.

In order to finally prove that $\Omega_{\mathrm{PA}}^{*}(X)$ is an algebra we need the following extension of Proposition 5.9 to PA forms.

Proposition 5.25 Let $X_{1}$ and $X_{2}$ be semi-algebraic sets and let $\alpha \in \Omega_{\mathrm{PA}}^{k}\left(X_{1} \times X_{2}\right)$. If $\left\langle\alpha, \gamma_{1} \times \gamma_{2}\right\rangle=0$ for all $\gamma_{1} \in \mathrm{C}_{*}\left(X_{1}\right)$ and $\gamma_{2} \in \mathrm{C}_{*}\left(X_{2}\right)$ then $\alpha=0$. 
The proof of this proposition is more complicated than for the case of minimal forms, in particular because it is unclear whether, for a PA form $\alpha$ on $X$, there exists a dense semi-algebraic smooth manifold on which $\alpha$ is smooth.

To prove Proposition 5.25, first we establish two lemmas. The first is an integral representation of PA forms. To state it, we introduce the following notation. Suppose that $X=(0,1)^{n}$ is an open $n$-cube and let $x_{1}, \ldots, x_{n}$ be the standard coordinates. For a subset $K=\left\{i_{1}, \ldots, i_{k}\right\} \subset \underline{n}=\{1, \ldots, n\}$ with $1 \leq i_{1}<\cdots<i_{k} \leq n$ we set

$$
d x_{K}=d x_{i_{1}} \wedge \cdots \wedge d x_{i_{k}}
$$

Lemma 5.26 Let $X=(0,1)^{n}$ and $\alpha \in \Omega_{\mathrm{PA}}^{k}(X)$. Assume that $\alpha$ is a trivial fiber integral over $X$. There exists a subset $X_{0} \subset X$ such that the complement $T=X \backslash X_{0}$ is a closed smoothly stratifiable set of codimension 1 and there exists, for each subset $K \subset \underline{n}$ of cardinality $k$, a smooth function $G_{K}: X_{0} \rightarrow \mathbb{R}$ satisfying the following: If $V$ is a semi-algebraic smooth oriented submanifold of dimension $k$ in $\mathbb{R}^{n}$ such that $\bar{V} \subset X_{0}$, then

$$
\langle\alpha, \llbracket V \rrbracket\rangle=\sum_{K} \int_{V} G_{K} d x_{K},
$$

where the sum runs over all subsets $K \subset \underline{n}$ of cardinality $k$.

Remark 5.27 Here by $\int_{V} G_{K} d x_{K}$ we mean $\int_{V} i^{*}\left(G_{K} d x_{K}\right)$, where $i: V \hookrightarrow X$ is the inclusion and $i^{*}$ is the pullback of differential forms.

Remark 5.28 Since $X_{0}$ may not be semi-algebraic, we cannot quite speak of the restriction $\left.\alpha\right|_{X_{0}}$, but we will nevertheless abuse notation and say that $\left.\alpha\right|_{X_{0}}$ is given by the integral representation (9). If we just ask for the integral representation without smoothness assumption on the $G_{K}$ 's then we can assume that $X_{0}$ is semi-algebraic (see formula (12) below which gives an integral representation on the semi-algebraic dense subset $X^{\prime}$ ).

Proof Since $\alpha$ is a trivial fiber integral over $X$ there exists a compact oriented semialgebraic manifold $Y$ of dimension $l$ and a minimal form $\mu \in \Omega_{\min }^{k+l}(X \times Y)$ such that $\alpha=f_{Y} \mu$. By taking a finite cubification of $Y$ it is easy to see that we can suppose that $Y=[0,1]^{l}$ (by replacing $\mu$ by the sum of the restrictions of $\mu$ over a finite partition "up to codimension 1 faces" of $Y$ into cubes $[0,1]^{l}$ ).

Now, $\mu=\sum_{j=1}^{s} \lambda\left(f_{0}^{j} ; f_{1}^{j}, \ldots, f_{k+l}^{j}\right)$ for $f_{i}^{j}: X \times Y \rightarrow \mathbb{R}$ semi-algebraic functions. There exists a codimension 1 semi-algebraic subset $Z \subset X \times Y$ on the complement of 
which the $f_{i}^{j}$ are smooth, and hence

$$
\left.\mu\right|_{(X \times Y) \backslash Z}=\sum_{j=1}^{s} f_{0}^{j} d f_{1}^{j} \ldots d f_{k+l}^{j} .
$$

Denote by $x_{1}, \ldots, x_{n}$ and $y_{1}, \ldots, y_{l}$ the coordinates on $X$ and $Y$. Then on $(X \times Y) \backslash Z$,

$$
\sum_{j=1}^{s} f_{0}^{j} d f_{1}^{j} \ldots d f_{k+l}^{j}=\sum_{K, L} g_{K, L} d y_{L} d x_{K}
$$

where the sum runs over subsets $K \subset \underline{n}$ and $L \subset \underline{l}$ with $|K|+|L|=k+l$ and $g_{K, L}$ are semi-algebraic smooth functions on $(X \times Y) \backslash Z\left(g_{K, L}\right.$ is a signed sum of $(k+l)$ ! products of $f_{0}^{j}$ with first order partial derivatives of $f_{i}^{j}$ for $\left.1 \leq i \leq k+l\right)$. When $K \subset \underline{n}$ is of cardinality $k$ then $L=\underline{l}$ and we set $g_{K}=g_{K, \underline{l}}$.

For $x \in X$, consider the slice

$$
Z(x)=\{y \in Y:(x, y) \in Z\} .
$$

Now set $T^{\prime}=\{x \in X: \operatorname{dim} Z(x)=l\}$. This is a semi-algebraic subset of $X$ as can be seen from the semi-algebraic local triviality theorem [1, Proposition 9.3.1] applied to pr: $Z \rightarrow X$. Further, $\operatorname{dim} T^{\prime}<n$ because $\operatorname{dim} Z<n+l$.

Fix $K \subset \underline{n}$ of cardinality $k$. For $x \in X \backslash T^{\prime}$, the function

$$
\begin{aligned}
g_{K}(x,-): Y & \longrightarrow \mathbb{R} \\
y & \longmapsto g_{K}(x, y)=g_{K, \underline{l}}(x, y)
\end{aligned}
$$

is almost everywhere defined and, by [1, Proposition 2.9.1 and remark that follows it], it is semi-algebraic on its domain. Set $g_{K}^{+}=\max \left(g_{K}, 0\right)$ and $g_{K}^{-}=g_{K}^{+}-g_{K}$. Consider the hypographs of $g_{K}^{ \pm}$,

$$
U_{K}^{ \pm}:=\left\{(x, y, t) \in((X \times Y) \backslash Z) \times \mathbb{R}: 0 \leq g_{K}^{ \pm}(x, y) \leq t\right\} .
$$

With analogous notation for the slice as in Equation (11), we thus have, for $x \in X \backslash T^{\prime}$,

$$
\mathcal{H}^{l+1}\left(U_{K}^{ \pm}(x)\right)=\int_{Y} g_{K}^{ \pm}(x, y) d y_{\underline{l}},
$$

and $g_{K}(x,-)$ is integrable if and only if $U_{K}^{+}(x)$ and $U_{K}^{-}(x)$ are of finite $(l+1)-$ volume. By [3, Theorem 3], the set

$$
T_{K}^{\prime \prime}:=\left\{x \in X \backslash T^{\prime}: g_{K}(x,-) \text { is not integrable over } Y\right\}
$$

is semi-algebraic. 
We now claim that $\operatorname{dim}\left(T_{K}^{\prime \prime}\right)<n$.

Let $\left\{i_{k+1}, \ldots, i_{n}\right\}$ be the complement of the set $K$ in $\underline{n}$ and define the top degree minimal form

$$
\mu_{K}=\sum_{j=1}^{s} \lambda\left(f_{0}^{j} ; f_{1}^{j}, \ldots, f_{k+l}^{j}, x_{i_{k+1}}, \ldots, x_{i_{n}}\right) \in \Omega_{\min }^{n+l}(X \times Y)
$$

which is also smooth on $(X \times Y) \backslash Z$. Using (10), we have that

$$
\pm \sum_{j=1}^{s} f_{0}^{j} d f_{1}^{j} \ldots d f_{k+l}^{j} d x_{i_{k+1}} \cdots d x_{i_{n}}=g_{K} d y_{\underline{l}} d x_{\underline{n}}
$$

where the sign comes from the signature of the permutation of the shuffle $(K, \underline{n} \backslash K)$. For concreteness, we will assume that this signature is +1 . Set

$$
A_{K}^{ \pm}=\left\{(x, y) \in X \times Y \backslash Z: g_{K}^{ \pm}(x, y)>0\right\} .
$$

To avoid issues arising from the noncompactness of the open cube $X$, fix $\epsilon>0$ and let $X_{\epsilon}=[\epsilon, 1-\epsilon]^{n}$ and $A_{K, \epsilon}^{ \pm}=A_{K}^{ \pm} \cap\left(X_{\epsilon} \times Y\right)$. Consider the chains $\llbracket A_{K, \epsilon}^{ \pm} \rrbracket \in \mathrm{C}_{n+l}(X \times Y)$ obtained by taking the $(n+l)$-dimensional strata of $A_{K, \epsilon}^{ \pm}$equipped with the orientation of $X \times Y$. Then

$$
\int_{X_{\epsilon} \times Y}\left|g_{K}(x, y)\right| d y_{\underline{l}} d x_{\underline{n}}=\left\langle\mu_{K}, \llbracket A_{K, \epsilon}^{+} \rrbracket\right\rangle-\left\langle\mu_{K}, \llbracket A_{K, \epsilon}^{-} \rrbracket\right\rangle<\infty,
$$

and hence $g_{K}$ is integrable over $X_{\epsilon} \times Y$. By Fubini's Theorem (and since $\epsilon>0$ is arbitrary), we deduce that for almost every $x \in X \backslash T^{\prime}, g_{K}(x,-)$ is integrable, which establishes the claim.

Now set

$$
X^{\prime}=X \backslash \overline{T^{\prime} \cup \bigcup_{K \subset \underline{n},|K|=k} T_{K}^{\prime \prime}} .
$$

This is an open dense semi-algebraic subset of $X$. For each $x \in X^{\prime}, g_{K}^{ \pm}(x,-)$ is integrable on $Y$ and we set

$$
\begin{aligned}
G_{K}^{ \pm}: X^{\prime} & \longrightarrow \mathbb{R} \\
x & \longmapsto \int_{Y} g_{K}^{ \pm}(x, y) d y_{\underline{l}}
\end{aligned}
$$

and $G_{K}=G_{K}^{+}-G_{K}^{-}$. If $V$ is a semi-algebraic $k$-dimensional oriented smooth submanifold with closure in $X^{\prime}$, then since $V \cap T^{\prime}=\varnothing$ we have that $Z \cap(V \times Y)$ is negligible in $V \times Y$ and using (10) we have

$$
\langle\alpha, \llbracket V \rrbracket\rangle=\int_{(V \times Y) \backslash Z} \sum_{K, L} g_{K, L} d y_{L} d x_{K} .
$$


If $|L|<l$ then $|K|>k$ and since $V$ is $k$-dimensional in the direction of the $x_{i}$ 's and $F$ is in the direction of the $y_{i}$ 's, we have that the restriction of the differential form $g_{K, L} d y_{L} d x_{K}$ to the tangent space of $(V \times Y) \backslash Z$ is zero. Therefore the only contributions come from the $g_{K}=g_{K}, \underline{l}$, and by Fubini's Theorem we have

$$
\langle\alpha, \llbracket V \rrbracket\rangle=\sum_{K} \int_{V} G_{K}(x) d x_{K} .
$$

It remains to prove that $G_{K}$ is smooth on the complement of a codimension 1 smoothly stratified closed subspace $T$ of $X$. The value of the integral $G_{K}(x)$, for $x \in X^{\prime}$, can be obtained as the difference of volumes of the slices at $x$ of the hypographs of $g_{K}^{+}$and $g_{K}^{-}$. These hypographs are semi-algebraic sets, hence they are global subanalytic sets (see [3] for a quick definition and a list of references on global subanalytic sets and functions). By Theorem 1' of [3] there exist (global) subanalytic functions $A_{1}, \ldots, A_{s}: X^{\prime} \rightarrow \mathbb{R}$ (not necessarily continuous) and a polynomial $P \in \mathbb{R}\left[a_{1}, \ldots, a_{s}, u_{1}, \ldots, u_{s}\right]$ such that

$$
G_{K}=P\left(A_{1}, \ldots, A_{s}, \log \left(A_{1}\right), \ldots, \log \left(A_{s}\right)\right) .
$$

By [14] (see also [10]), graphs of subanalytic functions are stratifiable, and therefore there exists a finite stratification of $X^{\prime}$ into subanalytic smooth submanifolds such that $G_{K}$ is smooth on each stratum. Let $X_{0}$ be the union of the maximal strata. Then $G_{K}$ is smooth on $X_{0}$ and its complement $T=X^{\prime} \backslash X_{0}$ is a closed smoothly stratifiable subset of codimension 1 .

We next establish a continuity principle for PA forms: A PA form is determined by its values on the complement of any closed codimension 1 smoothly stratifiable subset. We have an analogous statement for minimal forms in Proposition 5.8 but the proof there was easier because we assumed that the codimension 1 subset was semi-algebraic.

Lemma 5.29 Let $X$ be a semi-algebraic set, let $T \subset X$ be a closed codimension 1 smoothly stratifiable subset, and let $\alpha \in \Omega_{\mathrm{PA}}^{k}(X)$ be a PA form. If for all $\gamma \in \mathrm{C}_{k}(X)$,

$$
\operatorname{spt}(\gamma) \cap T=\varnothing \Longrightarrow\langle\alpha, \gamma\rangle=0,
$$

then $\alpha=0$.

Remark 5.30 This could be summarized by " $\left.\alpha\right|_{X \backslash T}=0 \Longrightarrow \alpha=0$ " but this does not quite make sense because $X \backslash T$ may be not semi-algebraic.

Proof Let $\gamma \in \mathrm{C}_{k}(X)$. We will prove that $\langle\alpha, \gamma\rangle=0$.

Consider a cubification of $X$ such that $\alpha$ is a trivial fiber integral over each closed cube and such that $\gamma$ is a linear combination of cubes. By linearity it is enough to 
prove the statement when $\gamma=\llbracket \bar{\kappa} \rrbracket$, where $\kappa$ is a single cube. Let $\tau$ be an open cube of maximal dimension such that $\kappa$ is a face of it. There exists some semi-algebraic homeomorphism which lets us assume that $\bar{\tau}=[0,1]^{n}$ and that $\bar{\kappa}=[0,1]^{k} \times\{0\}^{n-k}$. The image of $T$ under this semi-algebraic homeomorphism is still of codimension 1 .

Since $\alpha$ is a trivial fiber integral over $[0,1]^{n}$, there exists a compact oriented manifold $F$ of dimension $l$ and a minimal form

$$
\mu=\sum_{i=1}^{r} \lambda\left(f_{0}^{i} ; f_{1}^{i}, \ldots, f_{k+l}^{i}\right) \in \Omega_{\min }^{k+l}\left([0,1]^{n} \times F\right)
$$

such that $\left.\alpha\right|_{\bar{\tau}}=f_{\widehat{F}} \mu$. There exists $Z \subset[0,1]^{n} \times F$ of codimension 1 such that the $f_{j}^{i}$ are smooth on the complement of $Z$. As seen in the proof of Lemma 5.26 (the part following equation (11)), the set $T^{\prime}:=\left\{x \in[0,1]^{n}: \operatorname{dim}(Z \cap\{x\} \times F)=l\right\}$ is semi-algebraic of codimension 1 and the same is true for its closure. Since we can always enlarge $T$, we can assume that $\overline{T^{\prime}} \subset T$. This implies that if $V$ is a smooth submanifold in $X$ then the $f_{j}^{i}$,s are smooth almost everywhere on $(V \backslash T) \times F$.

We now claim that there exists a sequence $\left\{\gamma_{n}\right\}$ in $C^{k}(\bar{\tau})$ such that $\gamma_{n} \stackrel{\text { SA }}{\rightarrow} \gamma$ and $\mathcal{H}^{k}\left(\operatorname{spt}\left(\gamma_{n}\right) \cap T\right)=0$.

First recall that $\gamma=\llbracket[0,1]^{k} \times\{0\}^{n-k} \rrbracket$. To prove the claim, consider the positive orthant of the $(n-k-1)$-dimensional sphere

$$
S_{+}^{n-k-1}=\left\{v \in[0,1]^{n-k}:\|v\|=1\right\}
$$

and of the $(n-k)-$ disk

$$
D_{+}^{n-k}=\left\{v \in[0,1]^{n-k}:\|v\| \leq 1\right\} .
$$

Since $T$ is of codimension 1 in $[0,1]^{n}$, we have that $\mathcal{H}^{n}\left(T \cap\left([0,1]^{k} \times D_{+}^{n-k}\right)\right)=0$. Passing to polar coordinates we have

$$
\mathcal{H}^{n}\left(T \cap\left([0,1]^{k} \times D_{+}^{n-k}\right)\right)=C \cdot \int_{0}^{1} \int_{S_{+}^{n-k-1}} \mathcal{H}^{k}\left(T \cap[0,1]^{k} \times\{r v\}\right) r^{n-k-1} d v d r
$$

where $C$ is a positive constant, $d v$ is the standard $(n-k-1)$-area measure on $S_{+}^{n-k-1}$ and $d r$ is the Lebesgue measure on the interval $[0,1]$. Hence the double integral is zero and since the integrand is nonnegative, there exists $v_{0} \in S_{+}^{n-k-1}$ such that $\mathcal{H}^{k}\left(T \cap[0,1]^{k} \times\left\{r v_{0}\right\}\right)=0$ for almost every $r \in[0,1]$. Therefore there exists a sequence $\left\{r_{n}\right\}$ in $[0,1]$, decreasing to 0 , such that if we set

$$
\gamma_{n}=\llbracket[0,1]^{k} \times\left\{r_{n} \cdot v_{0}\right\} \rrbracket \in \mathrm{C}_{k}\left([0,1]^{n}\right)
$$

then $\mathcal{H}^{k}\left(T \cap \operatorname{spt}\left(\gamma_{n}\right)\right)=0$ and $\gamma_{n} \stackrel{\text { SA }}{\rightarrow} \gamma$. This proves the claim. 
Clearly $\gamma_{n} \times \llbracket F \rrbracket \stackrel{\text { AA }}{\rightarrow} \gamma \times \llbracket F \rrbracket$ and from Proposition 5.6 we have

$$
\langle\alpha, \gamma\rangle=\langle\mu, \gamma \times \llbracket F \rrbracket\rangle=\lim _{n \rightarrow \infty}\left\langle\mu, \gamma_{n} \times \llbracket F \rrbracket\right\rangle=\lim _{n \rightarrow \infty}\left\langle\alpha, \gamma_{n}\right\rangle .
$$

Therefore it is now enough to prove that $\langle\alpha, \gamma\rangle=0$ when $\mathcal{H}^{k}(T \cap \operatorname{spt}(\gamma))=0$, and from now on we suppose that $\operatorname{spt}(\gamma) \cap T$ has zero $k$-volume. We can moreover suppose that $\gamma=\llbracket V \rrbracket$, where $V$ is a semi-algebraic oriented smooth submanifold of dimension $k$.

Let $m \geq 1$. Since $T$ is closed, $\operatorname{spt}(\gamma) \cap T$ is compact and it is covered by finitely many open balls $B\left(x_{i}^{(m)}, 1 / m\right)$, centered at $x_{i}^{(m)} \in T$ and of radius $1 / m$, for $1 \leq i \leq N_{m}$. Set $V_{m}=V \backslash \bigcup_{i=1}^{N_{m}} B\left[x_{i}^{(m)}, 2 / m\right]$. This is a semi-algebraic oriented smooth submanifold. Since $\overline{V_{m}} \cap T=\varnothing$, the hypothesis of the lemma implies that $\left\langle\alpha, \llbracket V_{m} \rrbracket\right\rangle=0$. Using that the $f_{j}^{i}$ are smooth almost everywhere on $(V \backslash T) \times F$, that $\mathcal{H}^{k}(T \cap V)=0$, and that $\bigcup_{m \geq 1} V_{m}=V \backslash T$, as well as the Lebesgue Bounded Convergence Theorem, we get

$$
\begin{aligned}
\langle\alpha, \llbracket V \rrbracket\rangle & =\langle\mu, \llbracket V \times F \rrbracket\rangle \\
& =\sum_{i=1}^{r} \int_{(V \backslash T) \times F} f_{0}^{i} d f_{1}^{i} \cdots d f_{k+l}^{i} \\
& =\lim _{m \rightarrow \infty} \sum_{i=1}^{r} \int_{V_{m} \times F} f_{0}^{i} d f_{1}^{i} \cdots d f_{k+l}^{i} \\
& =\lim _{m \rightarrow \infty}\left\langle\alpha, \llbracket V_{m} \rrbracket\right\rangle \\
& =0 .
\end{aligned}
$$

Proof of Proposition 5.25 Let $\gamma \in \mathrm{C}_{k}\left(X_{1} \times X_{2}\right)$. We will prove that $\langle\alpha, \gamma\rangle=0$.

Because $\operatorname{spt}(\gamma)$ is compact, we are able to restrict to the product of compact sets $\operatorname{pr}_{1}(\operatorname{spt}(\gamma)) \times \operatorname{pr}_{2}(\operatorname{spt}(\gamma)) \subset X_{1} \times X_{2}$, so we can suppose that $X_{1}$ and $X_{2}$ are compact. Take cubifications $\left\{\kappa_{i}^{1}\right\}$ and $\left\{\kappa_{j}^{2}\right\}$ of $X_{1}$ and $X_{2}$. By linearity it is enough to check

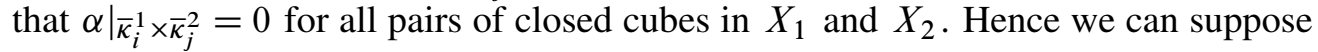
that $X_{1}=[0,1]^{n_{1}}$ and $X_{2}=[0,1]^{n_{2}}$.

Now take a triangulation of $X_{1} \times X_{2}$ such that $\alpha$ is a trivial fiber integral over the closure of each simplex and such that $\gamma$ is a linear combination of simplices. By linearity, without loss of generality we can assume that $\gamma=\llbracket \bar{\sigma} \rrbracket$ for a single simplex $\sigma$. Let $\tau$ be a maximal dimensional open simplex such that $\sigma$ is a face of $\bar{\tau}$. By the same argument as in the proof of Proposition 4.3, we can construct a sequence $\left\{\gamma_{n}\right\} \subset \mathrm{C}_{k}(\tau)$ such that $\gamma_{n} \stackrel{\text { SA }}{\rightarrow} \gamma$. By the same argument as in equation (13), it is enough to show that $\left\langle\alpha, \gamma_{n}\right\rangle=0$. In other words, there is no loss of generality in supposing that $\operatorname{spt}(\gamma)$ is 
contained in the simplex $\tau$ which is an open set in $X_{1} \times X_{2}$ (because it is of maximal dimension).

Since $\operatorname{spt}(\gamma)$ is compact, it is covered by finitely many open sets of the form $O_{1} \times O_{2}$ contained in $\tau$ and such that $O_{i}$ is an open set in $X_{i}$ semi-algebraically homeomorphic to $(0,1)^{n_{i}}$ for $i=1,2$. To conclude the proof, we will show that $\left.\alpha\right|_{O_{1} \times O_{2}}=0$.

Set $X=O_{1} \times O_{2} \cong(0,1)^{n}$ with $n=n_{1}+n_{2}$ and notice that $\alpha$ is a trivial fiber integral over $X$ since $X \subset \tau$. By Lemma 5.26, there exists a closed codimension 1 smoothly stratified $T \subset X$ and smooth functions $G_{K}: X \backslash T \rightarrow \mathbb{R}$ for $K \subset \underline{n}$ of cardinality $k$, giving the integral representation (9) for $\left.\alpha\right|_{X \backslash T}$. We prove that $G_{K}=0$. For concreteness, suppose that $K=\left\{1, \ldots, k_{1}, n_{1}+1, \ldots, n_{1}+k_{2}\right\}$ with $k_{1}+k_{2}=k$ and $0 \leq k_{i} \leq n_{i}$. If $G_{K}$ was not zero there would exist an $x \in X \backslash T$ such that, say, $G_{K}(x)>0$. By continuity, there exists $\epsilon>0$ and nonempty subsets $U_{1} \subset O_{1}$ and $U_{2} \subset O_{2}$ such that $\left.G_{K}\right|_{U_{1} \times U_{2}}>\epsilon$. Take $V_{1} \subset U_{1}$ a nonempty $k_{1}$-dimensional semialgebraic submanifold parallel to the linear space $[0,1]^{k_{1}} \times\{0\}^{n-k_{1}}$ (ie a codimension 0 manifold contained in some translate of $\left.[0,1]^{k_{1}} \times\{0\}^{n-k_{1}}\right)$ and similarly a nonempty $k_{2}$-dimensional manifold $V_{2} \subset U_{2}$ parallel to $\{0\}^{n_{1}} \times[0,1]^{k_{2}} \times\{0\}^{n_{2}-k_{2}}$. By Fubini's Theorem,

$$
\left\langle\alpha, \llbracket V_{1} \rrbracket \times \llbracket V_{2} \rrbracket\right\rangle=\int_{V_{1} \times V_{2}} G_{K} d x_{K} \geq \epsilon \cdot \mathcal{H}^{k}\left(V_{1} \times V_{2}\right)>0,
$$

which contradicts our main hypothesis. Thus $G_{K}=0$. This implies $\left.\alpha\right|_{\left(O_{1} \times O_{2}\right) \backslash T}=0$. By Lemma 5.29 we deduce that $\left.\alpha\right|_{O_{1} \times O_{2}}=0$ and the proposition is proved.

Proposition 5.31 Let $X_{1}$ and $X_{2}$ be semi-algebraic sets. There is a degree-preserving linear map

$$
\times: \Omega_{\mathrm{PA}}^{*}\left(X_{1}\right) \otimes \Omega_{\mathrm{PA}}^{*}\left(X_{2}\right) \longrightarrow \Omega_{\mathrm{PA}}^{*}\left(X_{1} \times X_{2}\right)
$$

characterized by the formula

$$
\left\langle\alpha_{1} \times \alpha_{2}, \gamma_{1} \times \gamma_{2}\right\rangle=\left\langle\alpha_{1}, \gamma_{1}\right\rangle \cdot\left\langle\alpha_{2}, \gamma_{2}\right\rangle
$$

for $\alpha_{i} \in \Omega_{\mathrm{PA}}^{*}\left(X_{i}\right)$ and $\gamma_{i} \in \mathrm{C}_{*}\left(X_{i}\right), i=1,2$. The Leibniz formula

$$
\delta\left(\alpha_{1} \times \alpha_{2}\right)=\delta\left(\alpha_{1}\right) \times \alpha_{2}+(-1)^{\operatorname{deg}\left(\alpha_{1}\right)} \alpha_{1} \times \delta\left(\alpha_{2}\right)
$$

also holds. Further, let $T: X_{2} \times X_{1} \rightarrow X_{1} \times X_{2}$ be the twisting map given by $\left(x_{2}, x_{1}\right) \mapsto$ $\left(x_{1}, x_{2}\right)$. Then

$$
\alpha_{2} \times \alpha_{1}=(-1)^{\operatorname{deg}\left(\alpha_{1}\right) \operatorname{deg}\left(\alpha_{2}\right)} T^{*}\left(\alpha_{1} \times \alpha_{2}\right)
$$


Proof We can write $\alpha_{i}=f_{\Phi_{i}} \mu_{i}$ for some $\mu_{i} \in \Omega_{\min }^{*}\left(Y_{i}\right)$ and $\Phi_{i} \in \mathrm{C}^{\mathrm{str}}\left(Y_{i} \rightarrow X_{i}\right)$, $i=1,2$. Set

$$
\alpha_{1} \times \alpha_{2}=f_{\Phi_{1} \times \Phi_{2}} \mu_{1} \times \mu_{2}
$$

It is straightforward to check that this PA form has the desired characterizing property. The Leibniz formula is a consequence of Lemma 5.23 and of the Leibniz formulas for minimal forms and continuous chains (Proposition 5.10 and Proposition 5.17.) Similarly for the twisting formula. The right side of Equation (15) is independent of the choice of the representatives of $\alpha_{i}$ by Proposition 5.25, which also implies that Equation (14) characterizes this cross product.

We define a multiplication on $\Omega_{\mathrm{PA}}^{*}(X)$ by

$$
\alpha_{1} \cdot \alpha_{2}=\Delta^{*}\left(\alpha_{1} \times \alpha_{2}\right)
$$

where $\Delta: X \rightarrow X \times X$ is the diagonal map. It is immediate from the previous proposition that this multiplication satisfies the Leibniz formula and is graded commutative.

In conclusion, we have:

Theorem 5.32 The above construction of PA forms defines a contravariant functor $\Omega_{\mathrm{PA}}^{*}:$ SemiAlg $\rightarrow$ CDGA.

\section{Equivalence between $A_{P L}$ and $\Omega_{\mathrm{PA}}^{*}$}

Recall that SemiAlg is the category of semi-algebraic sets. There is an obvious forgetful functor $u:$ SemiAlg $\rightarrow$ Top. Consider the contravariant functors

$$
\begin{array}{r}
\Omega_{\mathrm{PA}}^{*}: \text { SemiAlg } \longrightarrow \text { CDGA } \\
\mathrm{A}_{\mathrm{PL}}(u(-) ; \mathbb{R}): \text { SemiAlg } \longrightarrow \text { CDGA }
\end{array}
$$

where CDGA the category of commutative differential graded algebras and $A_{P L}$ is the Sullivan functor of piecewise polynomial forms as defined in [2] (see also [7]). Abusing notation we will simply write $X$ for $u(X)$. The aim of this section is to prove our main theorem:

Theorem 6.1 There is a zigzag of natural transformations

$$
\Omega_{\mathrm{PA}}^{*}(X) \longrightarrow \cdots \longleftarrow \mathrm{A}_{\mathrm{PL}}(X ; \mathbb{R})
$$

which is a weak equivalence when $X$ is a compact semi-algebraic set. 
The explicit chain of quasi-isomorphisms will be given in Section 6.4, Equation (20) before Proposition 6.11. The proof of this theorem will mimic the proof of [7, Section 11(d)] which gives a weak equivalence between $A_{P L}(-; \mathbb{R})$ and the de Rham functor $\Omega_{\mathcal{C}^{\infty}}^{*}$ of smooth differential forms on a smooth manifold. From now on we will also drop the field of coefficients $\mathbb{R}$ from the notation of $\mathrm{A}_{\mathrm{PL}}$.

Remark 6.2 The theorem is likely to be true without the compactness hypothesis. As it stands, the statement is sufficient for the application we have in mind (formality of the little cubes operad). Our proof would work also in the noncompact case if Poincare Lemma 6.3 below could be proved for noncompact collapsible polyhedra. In fact, Kontsevich and Soibelman claim that the theorem is true for an even more general class of spaces they call PA spaces [19, Definition 20].

In order to prove the weak equivalence $\Omega_{\mathrm{PA}}^{*} \simeq \mathrm{A}_{\mathrm{PL}}$, we first need to establish some homotopy properties of $\Omega_{\mathrm{PA}}^{*}$, namely:

(1) a Poincaré lemma stating that $\Omega_{\mathrm{PA}}^{*}\left(\Delta^{k}\right)$ is acyclic;

(2) a sheaf property, ie that $\Omega_{\mathrm{PA}}^{*}$ satisfies a Mayer-Vietoris sequence;

(3) the fact that the simplicial sets $\Omega_{\mathrm{PA}}^{k}\left(\Delta^{\bullet}\right)$ are "extendable"; this is used in establishing the Mayer-Vietoris property for the functor $A_{\mathrm{PA}}$ which is built from the PA forms on the simplex $\Delta^{k}$ in the same way as $\mathrm{A}_{\mathrm{PL}}$ is built from the polynomial forms on $\Delta^{k}$.

We establish these results in the next three sections.

\subsection{Poincaré Lemma for $\Omega_{\mathrm{PA}}^{*}$}

Recall the notion of a collapsible polyhedron from Section 2.2. The version of the "Poincaré lemma" that we need is the following.

Lemma 6.3 Let $X$ be a compact semi-algebraic set that is semi-algebraically homeomorphic to a collapsible polyhedron. Then $\mathrm{H}^{0}\left(\Omega_{\mathrm{PA}}^{*}(X)\right) \cong \mathbb{R}$ and $\mathrm{H}^{i}\left(\Omega_{\mathrm{PA}}^{*}(X)\right)=0$ for $i>0$.

As in the classical proof of the Poincare lemma, the strategy is to convert a geometric homotopy into a cochain homotopy and to deduce that the cochain complex is acyclic. But this approach is not as straightforward in our case because it is unclear whether the cochain homotopy operator maps PA forms to PA forms (see Section 9.3). 
Set $I=[0,1]$ and let $h: X \times I \rightarrow X$ be a semi-algebraic homotopy. Define a cochain homotopy operator

$$
\Theta_{h}: \mathrm{C}^{k}(X) \rightarrow \mathrm{C}^{k-1}(X)
$$

by, for $\lambda \in \mathrm{C}^{k}(X)$ and $\gamma \in \mathrm{C}_{k-1}(X)$,

$$
\left\langle\Theta_{h}(\lambda), \gamma\right\rangle=\left\langle\lambda, h_{*}(\gamma \times \llbracket I \rrbracket)\right\rangle .
$$

It is easy to check that

$$
\delta \Theta_{h} \pm \Theta_{h} \delta=(h(-, 1))^{*}-(h(-, 0))^{*} .
$$

If $\lambda$ is a minimal form then $\Theta_{h}(\lambda)=f_{\widehat{I}} h^{*}(\lambda)$ is a PA form, but in general it is not a minimal form as can be seen from an example analogous to that of Remark 5.12. We do not know whether $\Theta_{h}$ maps PA forms to PA forms but we will establish a weaker result in that direction. To state it we need the following:

Definition 6.4 Let $X$ be a semi-algebraic set and let $A \subset X$ be a closed semi-algebraic subset. We say that a semi-algebraic homotopy $h: X \times I \rightarrow X$ is supported on $A$ if $h(A \times I) \subset A$ and $h(x, t)=x$ for $x \in \overline{X \backslash A}$ and $t \in I$.

Lemma 6.5 Let $X$ be a semi-algebraic set, let $A \subset X$ be a closed semi-algebraic subset and let $h: X \times I \rightarrow X$ be a semi-algebraic homotopy. If $h$ is supported on $A$ and if $\alpha \in \Omega_{\mathrm{PA}}^{*}(X)$ is a trivial fiber integral over $A$, then $\Theta_{h}(\alpha) \in \Omega_{\mathrm{PA}}^{*}(X)$.

Proof The general idea of the proof is that on the one hand $\Theta_{h}(\alpha \mid \overline{X \backslash A})=0$ because the homotopy is trivial over $\overline{X \backslash A}$, and that on the other hand $\left.\alpha\right|_{A}=\int_{\widehat{F}} \mu$ so that $\Theta_{h}\left(\left.\alpha\right|_{A}\right)=\int_{F \times I}\left(h \times \mathrm{id}_{F}\right)^{*} \mu$. So $\Theta_{h}(\alpha)$ can be obtained by gluing two PA forms.

In more detail, set $X_{0}=\overline{X \backslash A}$ and $A_{0}=A \cap X_{0}$, so that $X$ is the pushout of $X_{0}$ and $A$ over $A_{0}$. We have $\alpha=\int_{\Phi} \mu$ where $\Phi \in \mathrm{C}_{l}^{\mathrm{str}}(f: Y \rightarrow X), \mu \in \Omega_{\min }^{k+l}(Y)$, and $A$ is the closure of a stratum of a trivialization of $\Phi$. Thus there exists a map $g: A \times F \rightarrow Y$ such that $F$ is a compact oriented manifold of dimension $l, f g=\mathrm{pr}_{1}$, and $g_{*}(\llbracket\{a\} \times F \rrbracket)=\Phi(a)$ for $a \in A$. Set $Y_{0}=f^{-1}\left(X_{0}\right)$. The map $g$ restricts to a map $g_{0}: A_{0} \times F \rightarrow Y_{0}$. Consider the pushout diagram

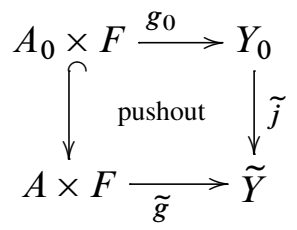

and the map $q: \tilde{Y} \rightarrow Y$ induced by $g: A \times F \rightarrow Y$ and $Y_{0} \hookrightarrow Y$. 
It is easy to construct a continuous chain $\widetilde{\Phi} \in \mathrm{C}_{l}^{\mathrm{str}}(f q: \tilde{Y} \rightarrow X)$ such that $q_{*}(\widetilde{\Phi}(x))=$ $\Phi(x)$ for $x \in X$, and hence $f_{\Phi} \mu=f_{\widetilde{\Phi}} q^{*}(\mu)$.

Now define a homotopy $\tilde{h}: \tilde{Y} \times I \rightarrow \tilde{Y}$ using maps $\widetilde{g}$ and $\tilde{j}$ from diagram (17) by

$$
\left\{\begin{aligned}
\tilde{h}(\tilde{g}(a, f), t) & =\widetilde{g}(h(a, t), f) & & \text { for } a \in A, f \in F \text { and } t \in I, \\
\widetilde{h}(\tilde{j}(y), t) & =\widetilde{j}(y) & & \text { for } y \in Y_{0} \text { and } t \in I .
\end{aligned}\right.
$$

That $\tilde{h}$ is well-defined is a consequence of the support of $h$ on $A$.

We will show that

$$
\Theta_{h}\left(f_{\Phi} \mu\right)=f_{\tilde{\Phi} \times \llbracket I \rrbracket} \tilde{h}^{*}\left(q^{*} \mu\right)
$$

which implies the statement of the lemma.

When restricted to $X_{0}$, the right side of Equation (18) is

$$
f_{\left(\left.\widetilde{\Phi}\right|_{X_{0}}\right) \times \llbracket \rrbracket}\left(\left.\tilde{h}\right|_{Y_{0} \times I} ^{*} q^{*}\right) \mu=f_{\left(\left.\tilde{\Phi}\right|_{X_{0}}\right) \times \llbracket I \rrbracket} \operatorname{pr}_{1}^{*} q^{*} \mu
$$

which is 0 because the projection $\mathrm{pr}_{1}$ decreases the dimension of the chains, and for the left side we have $\left.\Theta_{h}\right|_{X_{0} \times I}=0$ because the homotopy is constant over $X_{0}$.

On the other hand, for $\gamma \in \mathrm{C}_{k-1}(A)$, we have

$$
\begin{aligned}
\left\langle\Theta_{h}\left(f_{\Phi} \mu\right), \gamma\right\rangle & =\left\langle f_{\left.\Phi\right|_{A}}\left(\left.\mu\right|_{f^{-1}(A)}\right), h_{*}(\gamma \times \llbracket I \rrbracket)\right\rangle \\
& =\left\langle f_{\widehat{F}} g^{*}(\mu), h_{*}(\gamma \times \llbracket I \rrbracket)\right\rangle \\
& =\left\langle g^{*} \mu, h_{*}(\gamma \times \llbracket I \rrbracket) \times \llbracket F \rrbracket\right\rangle \\
& =\left\langle g^{*} \mu,\left(h \times \operatorname{id}_{F}\right)_{*}(\gamma \times \llbracket I \times F \rrbracket)\right\rangle \\
& =\left\langle\left(h \times \operatorname{id}_{F}\right)^{*}\left(g^{*} \mu\right), \gamma \times \llbracket I \times F \rrbracket\right\rangle \\
& =\left\langle f_{\tilde{\Phi} \times \llbracket I \rrbracket} \tilde{h}^{*}\left(q^{*} \mu\right), \gamma\right\rangle
\end{aligned}
$$

and this proves Equation (18) when restricted to $A$.

Since $\mathrm{C}_{*}(X)=\mathrm{C}_{*}(A)+\mathrm{C}_{*}\left(X_{0}\right)$, Equation (18) holds on $\mathrm{C}_{*}(X)$ and the lemma is proved.

We come to the proof of the Poincaré Lemma. 
Proof of Lemma 6.3 Since $X$ is collapsible, it is semi-algebraically path-connected and the only degree $0 \delta$-cocycles are the constant functions. So $\Omega_{\mathrm{PA}}^{0}(X) \cap \operatorname{ker} \delta \cong \mathbb{R}$.

Let $\alpha \in \Omega_{\mathrm{PA}}^{k}(X) \cap \operatorname{ker} \delta$ with $k \geq 1$. We will show that $\alpha \in \delta\left(\Omega_{\mathrm{PA}}^{k-1}(X)\right)$. We have $\alpha=f_{\Phi} \mu$ for some $\Phi \in \mathrm{C}_{l}^{\mathrm{str}}(Y \rightarrow X)$ and $\mu \in \Omega_{\min }^{k+l}(Y)$. Take a triangulation of $X$ trivializing $\Phi$. By the semi-algebraic Hauptvermutung (Theorem 2.6 and Corollary 2.7), the polyhedron associated to that triangulation is also collapsible, and it thus admits a subdivided triangulation that simplicially collapses to some vertex $*$. This means that the triangulation consists of closed simplices $\tau_{1}, \ldots, \tau_{N}$ such that if we set $T_{p}=$ $\bigcup_{i=p}^{N} \tau_{i}$ for $1 \leq p \leq N$ and $T_{N+1}=*$, then there exist semi-algebraic homotopies $h_{p}: T_{p} \times I \rightarrow T_{p}$ supported on $\tau_{p}$ such that $h_{p}(-, 0)=\mathrm{id}_{T_{p}}$ and $h_{p}\left(T_{p} \times\{1\}\right) \subset T_{p+1}$. Since $\left.\alpha\right|_{T_{p}}$ is a trivial fiber integral over $\tau_{p}$, Lemma 6.5 implies that $\Theta_{h_{p}}\left(\left.\alpha\right|_{T_{p}}\right) \in$ $\Omega_{\mathrm{PA}}^{*}\left(T_{p}\right)$. Define $g_{p}: T_{p} \rightarrow T_{p+1}$ by $g_{p}(x)=h_{p}(x, 1)$ and Equation (16) then implies that $\delta \Theta_{h_{p}}\left(\left.\alpha\right|_{T_{p}}\right)=g_{p}^{*}\left(\left.\alpha\right|_{T_{p+1}}\right)-\left.\alpha\right|_{T_{p}}$ for $1 \leq p \leq N$.

Set

$$
\begin{aligned}
\beta=\Theta_{h_{1}}\left(\left.\alpha\right|_{T_{1}}\right)+g_{1}^{*}\left(\Theta_{h_{2}}\left(\left.\alpha\right|_{T_{2}}\right)\right)+g_{1}^{*} g_{2}^{*}\left(\Theta_{h_{3}}\left(\left.\alpha\right|_{T_{3}}\right)\right) & \\
& +\cdots+g_{1}^{*} g_{2}^{*} \cdots g_{N-1}^{*}\left(\Theta_{h_{N}}\left(\left.\alpha\right|_{T_{N}}\right)\right) .
\end{aligned}
$$

This is an elements of $\Omega_{\mathrm{PA}}^{k-1}(X)$. Most of the terms of $\delta \beta$ cancel in pairs and we are left with

$$
\delta \beta=-\alpha+g_{1}^{*} g_{2}^{*} \cdots g_{N-1}^{*} g_{N}^{*}\left(\left.\alpha\right|_{T_{N+1}}\right) .
$$

The second term in this sum is 0 because $T_{N+1}=*$ and $\alpha$ is of positive degree. This proves that $\alpha=\delta(-\beta)$ is a coboundary in $\Omega_{\mathrm{PA}}^{*}(X)$.

\subsection{Sheaf propery of $\Omega_{\mathrm{PA}}^{*}$}

Definition 6.6 An excisive semi-algebraic pair is a pair $\left\{X_{1}, X_{2}\right\}$ of semi-algebraic sets in the same $\mathbb{R}^{m}$ such that there exist semi-algebraic functions $\rho_{i}: X_{1} \cup X_{2} \rightarrow[0,1]$ with $X_{i} \supset \rho_{i}^{-1}((0,1])$ for $i=1,2$ and $\rho_{1}+\rho_{2}=1$.

Lemma 6.7 Let $\left\{X_{1}, X_{2}\right\}$ be an excisive semi-algebraic pair. There exists an exact sequence

$$
0 \longrightarrow \Omega_{\mathrm{PA}}^{k}\left(X_{1} \cup X_{2}\right) \stackrel{r}{\longrightarrow} \Omega_{\mathrm{PA}}^{k}\left(X_{1}\right) \oplus \Omega_{\mathrm{PA}}^{k}\left(X_{2}\right) \stackrel{\Delta}{\longrightarrow} \Omega_{\mathrm{PA}}^{k}\left(X_{1} \cap X_{2}\right) \longrightarrow 0
$$

with $r(\alpha)=\left(\left.\alpha\right|_{X_{1}},\left.\alpha\right|_{X_{2}}\right)$ and $\Delta\left(\alpha_{1}, \alpha_{2}\right)=\left.\alpha_{1}\right|_{X_{1} \cap X_{2}}-\left.\alpha_{2}\right|_{X_{1} \cap X_{2}}$. 
Proof Set $X=X_{1} \cup X_{2}$. It is clear that $\Delta r=0$. It is also not difficult to construct, from the given partition of unity, another one that we will also denote by $\left\{\rho_{1}, \rho_{2}\right\}$ with smaller support and such that there exist semi-algebraic functions $\sigma_{i}: X \rightarrow[0,1]$ with $\rho_{i}(x)>0 \Longrightarrow \sigma_{i}(x)=1$ and $\overline{\sigma_{i}^{-1}((0,1])} \subset X_{i}$. This can be done by taking a semialgebraic function $\phi:[0,1] \rightarrow[0,1]$ such that $\phi([0,1 / 4])=\{0\}$ and $\phi(1-t)=$ $1-\phi(t)$, and composing the given $\rho_{i}$ with $\phi$ to get the new partition of unity. This leaves enough room in $X_{i} \cap \rho_{i}^{-1}(0)$ to build the desired functions $\sigma_{i}$. For the rest of the proof we will assume that we are dealing with this better partition of unity.

Note $X$ is the union of the closed sets $\rho_{i}^{-1}([1 / 4,1]), i=1$, 2. Since $\rho_{i}^{-1}([1 / 4,1]) \subset X_{i}$ we deduce that $\mathrm{C}_{k}(X)=\mathrm{C}_{k}\left(X_{1}\right)+\mathrm{C}_{k}\left(X_{2}\right)$. Hence $r$ is injective.

To prove the surjectivity of $\Delta$, let $\alpha \in \Omega_{\mathrm{PA}}^{k}\left(X_{1} \cap X_{2}\right)$ and let $j_{i}: X_{1} \cap X_{2} \hookrightarrow X_{i}$ be the inclusion. Then $\left.\alpha=\Delta\left(j_{1}^{*}\left(\rho_{2}\right) \alpha,-j_{2}^{*}\left(\rho_{1}\right) \alpha\right)\right)$.

It remains to prove that $\operatorname{ker} \Delta \subset \operatorname{im} r$. Let $\alpha_{i} \in \Omega_{\mathrm{PA}}^{k}\left(X_{i}\right)$ for $i=1,2$ be such that $\Delta\left(\alpha_{1}, \alpha_{2}\right)=0$. Let $\Phi_{i}: \mathrm{C}_{l}^{\mathrm{str}}\left(p_{i}: Y_{i} \rightarrow X_{i}\right)$ and $\mu_{i} \in \Omega_{\min }^{k+l}\left(Y_{i}\right)$ be such that $\alpha_{i}=f_{\Phi_{i}} \mu_{i}$ for $i=1,2$. There is no loss of generality in assuming that $l$ is positive and independent of $i$ as seen in the proof of Proposition 5.22. We can also assume that $Y_{i} \subset \mathbb{R}^{N}$.

Consider the following semi-algebraic sets and maps

$$
\begin{aligned}
& \tilde{Y}_{i}=\left\{\left(p_{i}(y), \sigma_{i}\left(p_{i}(y)\right) y\right): y \in Y_{i}\right\} \subset X \times \mathbb{R}^{N}, \\
& \tilde{p}_{i}: \tilde{Y}_{i} \rightarrow X \text { given by }(x, v) \mapsto x, \\
& q_{i}: Y_{i} \rightarrow \tilde{Y}_{i} \text { given by } y \mapsto\left(p_{i}(y), \sigma_{i}\left(p_{i}(y)\right) y\right) .
\end{aligned}
$$

Note the restriction of $q_{i}$ to $p_{i}^{-1}\left(\sigma_{i}^{-1}((0,1])\right.$ is a homeomorphism onto its image and that $q_{i}$ maps the entire fiber of $p_{i}$ over a point $x \in \sigma_{i}^{-1}(0)$ to a single point $\{(x, 0)\}$.

Define $\widetilde{\Phi}_{i}$ by

$$
\widetilde{\Phi}_{i}(x)= \begin{cases}q_{i *}\left(\Phi_{i}(x)\right) & \text { if } x \in X_{i}, \\ 0 & \text { if } \sigma_{i}(x)=0 .\end{cases}
$$

This defines a continuous chain $\widetilde{\Phi}_{i} \in \mathrm{C}_{l}^{\mathrm{str}}\left(\tilde{Y}_{i} \rightarrow X\right)$. It is also easy to build minimal forms $\tilde{\mu}_{i} \in \Omega_{\min }^{k+l}\left(\tilde{Y}_{i}\right)$ such that $q_{i}^{*}\left(\tilde{\mu}_{i}\right)=p_{i}^{*}\left(\rho_{i}\right) \mu_{i}$ in $\Omega_{\min }^{k+l}\left(Y_{i}\right)$.

Set $\tilde{Y}=\tilde{Y}_{1} \sqcup \tilde{Y}_{2}, \tilde{p}=\left(\tilde{p}_{1}, \tilde{p}_{2}\right): \tilde{Y} \rightarrow X, \tilde{\Phi}=\widetilde{\Phi}_{1}+\widetilde{\Phi}_{2} \in \mathrm{C}_{l}^{\operatorname{str}}(\tilde{Y} \rightarrow X)$, and $\tilde{\mu}=\tilde{\mu}_{1}+\tilde{\mu}_{2} \in \Omega_{\min }^{k+l}(\tilde{Y})$. This determines a PA form $\alpha=f_{\tilde{\Phi}} \tilde{\mu} \in \Omega_{\mathrm{PA}}^{k}(X)$ and one can easily check that $\left.\alpha\right|_{X_{i}}=\alpha_{i}$, since the left side of this equation can be identified (with abuse of notation) with $\left(\left.\alpha_{1} \cdot \rho_{1}\right|_{X_{i}}+\left.\alpha_{2} \cdot \rho_{2}\right|_{X_{i}}\right)$. 


\subsection{Extendability of the simplicial set $\Omega_{\mathrm{PA}}\left(\Delta^{\bullet}\right)$}

Definition 6.8 [7, page 118] A simplicial set $A_{\bullet}$ is extendable if, for each $n \geq 0$ and for each subset $I \subset\{0, \ldots, n\}$, given a collection $\beta_{i} \in A_{n-1}$ for $i \in I$ such that $\partial_{i} \beta_{j}=\partial_{j-1} \beta_{i}$ for each $i<j$ in $I$, there exists $\beta \in A_{n}$ such that $\beta_{i}=\partial_{i}(\beta)$ for all $i \in I$.

This section's goal is to prove the extendability of the simplicial vector spaces $\Omega_{\mathrm{PA}}^{k}\left(\Delta^{\bullet}\right)$.

Fix an integer $n$. Consider the "corner"

$$
X=\left\{x=\left(x_{1}, \ldots, x_{n}\right) \in \mathbb{R}^{n}: x_{i} \geq 0 \text { and } \sum_{i=1}^{n} x_{i} \leq 1\right\}
$$

whose codimension 1 faces containing the origin are given by

$$
\partial_{i} X=\left\{x \in X: x_{i}=0\right\} \quad \text { for } 1 \leq i \leq n .
$$

Notice that $\partial_{i} X$ consists of all the faces of $X$ except the "diagonal face" $\left\{\sum x_{i}=1\right\}$. The space $X$ is homeomorphic to the simplex $\Delta^{n}$. We next show that a family of compatible PA forms given on the non-diagonal faces can be extended into a PA form on $X$.

Lemma 6.9 Let $\alpha_{i} \in \Omega_{\mathrm{PA}}\left(\partial_{i} X\right)$ for $1 \leq i \leq n$. If $\left.\alpha_{i}\right|_{\partial_{i} X \cap \partial_{j} X}=\left.\alpha_{j}\right|_{\partial_{i} X \cap \partial_{j} X}$ for all $1 \leq i, j \leq n$ then there exists $\alpha \in \Omega_{\mathrm{PA}}(X)$ such that $\left.\alpha\right|_{\partial_{i} X}=\alpha_{i}$ for all $1 \leq i \leq n$.

Proof For $I \subset \underline{n}$, define $\partial_{I} X=\bigcap_{i \in I} \partial_{i} X$. In particular $\partial_{\varnothing} X=X$, and define $\operatorname{pr}_{I}: X \rightarrow \partial_{I} X$ by $\operatorname{pr}_{I}\left(x_{1}, \ldots, x_{n}\right)=\left(x_{1}^{\prime}, \ldots, x_{n}^{\prime}\right)$ with $x_{i}^{\prime}=0$ if $i \in I$ and $x_{i}^{\prime}=x_{i}$ otherwise. For $J \subset I$ consider also the inclusion maps $j_{J}^{I}: \partial_{I} X \rightarrow \partial_{J} X$.

Define

$$
\alpha=\sum_{\varnothing \neq I \subset \underline{n}}(-1)^{|I|-1} \operatorname{pr}_{I}^{*} j_{\{i\}}^{I *}\left(\alpha_{i}\right) \in \Omega_{\mathrm{PA}}^{*}(X)
$$

where $i$ is an arbitrary element of $I$. Using the hypothesis, one can easily check that $\alpha$ is independent of the choices of $i \in I$. Moreover,

$$
\begin{aligned}
\left.\alpha\right|_{\partial_{i} X}= & j_{\varnothing}^{\{i\} *} \alpha \\
= & j_{\varnothing}^{\{i\} *} \operatorname{pr}_{\{i\}}^{*} j_{\{i\}}^{\{i\} *}\left(\alpha_{i}\right) \\
& +\sum_{\varnothing \neq J \subset(n \backslash\{i\})}(-1)^{|J|-1}\left\{j_{\varnothing}^{\{i\} *} \operatorname{pr}_{J}^{*} j_{\{j\}}^{J *} \alpha_{j}-j_{\varnothing}^{\{i\} *} \operatorname{pr}_{J \cup\{i\}}^{*} j_{\{j\}}^{J \cup\{i\} *} \alpha_{j}\right\}
\end{aligned}
$$


where $j$ is an arbitrary element in $J$. Since the diagram

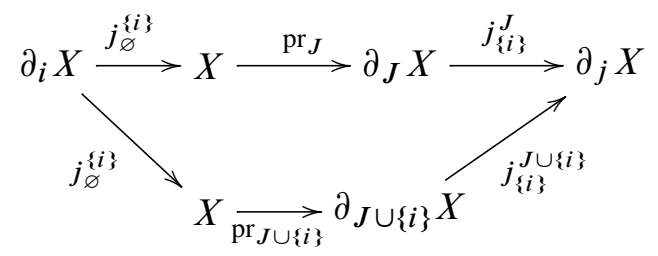

is commutative, it follows that all the terms in the brackets in the above sum vanish. Therefore $\left.\alpha\right|_{\partial_{i} X}=j_{\varnothing}^{\{i\} *} \operatorname{pr}_{\{i\}}^{*} j_{\{i\}}^{\{i\} *}\left(\alpha_{i}\right)=\alpha_{i}$.

Lemma 6.10 The simplicial vector space $\Omega_{\mathrm{PA}}^{k}\left(\Delta^{\bullet}\right)$ is extendable.

Proof By an elementary induction, it is enough to check the extendability condition of Definition 6.8 for $I=\{0, \ldots, n\}$.

Consider the standard $n$-simplex $\Delta^{n}=\left\{t=\left(t_{0}, \ldots, t_{n}\right) \in \mathbb{R}^{n+1}: t_{p} \geq 0, \sum_{p=0}^{n} t_{p}=1\right\}$. Its faces are $\partial_{p} \Delta^{n}=\left\{t \in \Delta^{n}: t_{p}=0\right\}$ for $0 \leq p \leq n$. Suppose given $\beta_{p} \in \Omega_{\mathrm{PA}}^{k}\left(\partial_{p} \Delta^{n}\right)$ for $0 \leq p \leq n$ such that $\left.\beta_{p}\right|_{\partial_{p} \Delta^{n} \cap \partial_{q} \Delta^{n}}=\left.\beta_{q}\right|_{\partial_{p} \Delta^{n} \cap \partial_{q} \Delta^{n}}$ for $0 \leq p, q \leq n$. We want to build $\beta \in \Omega_{\mathrm{PA}}^{k}\left(\Delta^{n}\right)$ such that $\beta_{p}=\left.\beta\right|_{\partial_{p} \Delta^{n}}$ for $0 \leq p \leq n$.

For $0 \leq p \leq n$ we have a semi-algebraic homeomorphism

$$
\begin{aligned}
\pi_{p}: \Delta^{n} & \stackrel{\cong}{\longrightarrow} X \\
\left(t_{0}, \ldots, t_{n}\right) & \longmapsto\left(x_{1}=t_{0}, \ldots, x_{p}=t_{p-1}, x_{p+1}=t_{p+1} \ldots, x_{n}=t_{n}\right)
\end{aligned}
$$

which corresponds to the orthogonal projection of the simplex onto the hyperplane $t_{p}=0$. This homeomorphism sends the faces of the simplex to the faces of $X$ with

$$
\pi_{p}\left(\partial_{i} \Delta^{n}\right)= \begin{cases}\partial_{i+1} X & \text { if } i<p \\ \partial_{i} X & \text { if } i>p\end{cases}
$$

and $\pi_{p}\left(\partial_{p} \Delta^{n}\right)$ is the "diagonal face" of $X$. We define PA forms $\alpha_{i}^{p} \in \Omega_{\mathrm{PA}}^{l}\left(\partial_{i} X\right)$ by

$$
\alpha_{i}^{p}= \begin{cases}\left(\pi_{p}^{-1}\right)^{*}\left(\beta_{i-1}\right) & \text { if } 1 \leq i<p, \\ \left(\pi_{p}^{-1}\right)^{*}\left(\beta_{i}\right) & \text { if } p+1 \leq i \leq n .\end{cases}
$$

By Lemma 6.9, there exists $\alpha^{p} \in \Omega_{\mathrm{PA}}^{l}(X)$ such that $\alpha^{p} \mid \partial_{i} X=\alpha_{i}^{p}$. We deduce that $\pi_{p}^{*}\left(\alpha^{p}\right) \mid \partial_{i} \Delta^{n}=\beta_{i}$ if $i \neq p$. Define a map $\tau_{p}: \Delta^{n} \rightarrow \mathbb{R}$ by $\tau_{p}\left(t_{0}, \ldots, t_{n}\right)=t_{p}$ and set

$$
\beta=\sum_{p=0}^{n} \tau_{p} \cdot \pi_{p}^{*}\left(\alpha^{p}\right) .
$$

Since $\sum \tau_{p}=1$ and $\tau_{p} \mid \partial_{p} \Delta^{n}=0$ it follows that $\beta \mid \partial_{p} \Delta^{n}=\beta_{p}$. 


\subsection{The weak equivalence $\Omega_{\mathrm{PA}}^{*} \simeq \mathrm{A}_{\mathrm{PL}}$}

We are ready to prove our main theorem by following the scheme of the proof of $\Omega_{C^{\infty}} \simeq A_{P L}$ from [7, Section 11(d)].

The geometric simplices define a semi-algebraic cosimplicial space $\left\{\Delta^{p}\right\}_{p \geq 0}$. Therefore we can consider the simplicial CDGA

$$
\mathrm{A}_{\mathrm{PA} \bullet}=\left\{\Omega_{\mathrm{PA}}^{*}\left(\Delta^{p}\right)\right\}_{p \geq 0} .
$$

This should be compared with the simplicial CDGA $\left(\mathrm{A}_{\mathrm{PL}}\right) \bullet$ from [7, Section 10(c)] where $A_{P L} p$ consists of polynomial forms on the simplicial set $\Delta_{\bullet}^{p}$. More precisely, this is the CDGA

$$
\mathrm{A}_{\mathrm{PL} p}=\bigwedge\left(t_{0}, \ldots, t_{p}, d t_{0}, \ldots, d t_{p}\right) /\left(1-\sum_{i=0}^{p} t_{i}, \sum_{i=0}^{p} d t_{i}\right) .
$$

Since a polynomial is a semi-algebraic function, there is also an obvious inclusion

$$
\mathrm{A} P\left\llcorner\mathrm{~A}_{\mathrm{PA}} \bullet\right.
$$

sending the polynomial $k$-form $\sum_{I=\left(i_{1}, \ldots, i_{k}\right) \subset[n]=\{0, \ldots, n\}} P_{I}\left(t_{0}, \ldots, t_{n}\right) d t_{i_{1}} \wedge \cdots \wedge d t_{i_{k}}$ to the minimal form $\sum_{I} \lambda\left(P_{I}\left(t_{0}, \ldots, t_{n}\right) ; t_{i_{1}}, \ldots, t_{i_{k}}\right)$ for $P_{I} \in \mathbb{R}\left[t_{0}, \ldots, t_{n}\right]$.

Recall that, as explained in [7, Section 10 (b)], to any such simplicial CDGA $A \bullet$ one associates a contravariant functor

$$
\begin{aligned}
A(-): \mathrm{sSet} & \longrightarrow \mathrm{CDGA} \\
S_{\bullet} & \longmapsto A\left(K_{\bullet}\right)=\operatorname{hom}_{\mathrm{sSet}}\left(K_{\bullet}, A_{\bullet}\right) .
\end{aligned}
$$

An element of $A^{k}\left(K_{\bullet}\right)$ can be regarded as a family $\left\{\Phi_{\sigma}\right\}_{\sigma \in K_{\bullet}}$ with $\Phi_{\sigma} \in A_{\operatorname{deg}(\sigma)}^{k}$, compatible with boundaries and degeneracies. In particular, we have the contravariant functors $A_{P L}$ and $A_{P A}$ from simplicial sets to CDGAs.

For a semi-algebraic set $X$, define the simplicial set of semi-algebraic singular simplices in $X$,

$$
\mathrm{S}_{\bullet}^{\mathrm{PA}}(X)=\left\{\mathrm{S}_{p}^{\mathrm{PA}}(X)\right\}_{p \geq 0}
$$

where

$$
\mathrm{S}_{p}^{\mathrm{PA}}(X)=\left\{\sigma: \Delta^{p} \rightarrow X \mid \sigma \text { is a semi-algebraic map }\right\} .
$$

We also have the classical simplicial set of singular simplices in $X, \mathrm{~S}_{\bullet}^{\text {sing }}(X)$, as in [7, Section 10(a) and Section 4 (a)] (where it is denoted by $S_{*}(X)$.) Since a semi-algebraic map $\sigma: \Delta^{p} \rightarrow X$ is continuous, we have a natural inclusion

$$
\mathrm{S}_{\bullet}^{\mathrm{PA}}(X) \hookrightarrow \mathrm{S}_{\bullet}^{\operatorname{sing}}(X) .
$$


Our equivalence between $\Omega_{\mathrm{PA}}^{*}$ and APL goes through the following natural zigzag

$$
\text { (20) } \Omega_{\mathrm{PA}}^{*}(X) \stackrel{\alpha_{X}}{\longrightarrow} \mathrm{A}_{\mathrm{PA}}\left(\mathrm{S}_{\bullet}^{\mathrm{PA}}(X)\right) \stackrel{\beta_{X}}{\longleftarrow} \mathrm{A}_{\mathrm{PL}}\left(\mathrm{S}_{\bullet}^{\mathrm{PA}}(X)\right) \stackrel{\gamma_{X}}{\longleftarrow} \mathrm{A}_{\mathrm{PL}}\left(\mathrm{S}_{\bullet}^{\text {sing }}(X)\right)=: \mathrm{A}_{\mathrm{PL}}(X)
$$

where, for $\omega \in \Omega_{\mathrm{PA}}(X), \alpha_{X}(\omega)=\left\{\sigma^{*}(\omega)\right\}_{\left\{\sigma \in \mathrm{S}_{\bullet}^{\mathrm{PA}}(X)\right\}}, \beta_{X}$ is induced by the inclusion $\mathrm{A}_{\mathrm{PL} \bullet} \hookrightarrow \mathrm{A}_{\mathrm{PA} \bullet}$, and $\gamma_{X}$ by the inclusion $\mathrm{S}_{\bullet}^{\mathrm{PA}}(X) \hookrightarrow \mathrm{S}_{\bullet}^{\text {sing }}(X)$.

The main idea for proving that these natural maps are weak equivalences is to use the following "Eilenberg-Steenrod criterion".

Proposition 6.11 Let $A, B$ : SemiAlg $\rightarrow \mathrm{Ch}^{*}(\mathbb{R})$ be two contravariant functors with values in cochain complexes and let $\theta: A \rightarrow B$ be a natural transformation between them. Suppose the following:

(1) If $X$ is empty or if $X$ is semi-algebraically homeomorphic to a collapsible polyhedron, then $\theta_{X}$ is a quasi-isomorphism.

(2) If $\left\{X_{1}, X_{2}\right\}$ is an excisive semi-algebraic pair and if $\theta_{X_{1}}, \theta_{X_{2}}$, and $\theta_{X_{1} \cap X_{2}}$ are quasi-isomorphisms, then so is $\theta_{X_{1} \cup X_{2}}$.

Then $\theta_{X}$ is a quasi-isomorphism for every compact semi-algebraic set $X$.

Proof As $X$ is a compact semi-algebraic set, after triangulating we can assume that it is a finite simplicial complex (by abuse of notation we will not distinguish a simplicial complex from its geometric realization). Consider its second barycentric subdivision $X^{\prime \prime}$. For each closed simplex $\sigma$ of $X$, we consider its second derived neighborhood, $N(\sigma)$, as defined in [16, page 50]. In more detail, $N(\sigma)$ is constructed as follows: If $v$ is a vertex of $X^{\prime \prime}$ then its star in $X^{\prime \prime}$ is the smallest closed simplicial subcomplex of $X^{\prime \prime}$ that is a topological neighborhood of $v$ in $X^{\prime \prime}$. In other words, the star of $v$ is obtained as the union of the closure of the simplices of $X^{\prime \prime}$ whose $v$ is a vertex. Then $N(\sigma)$ is defined to be the union of the stars of all the vertices of the second subdivision of $\sigma$, so it is also the smallest closed subcomplex of $X^{\prime \prime}$ which is a neighborhood of $\sigma$ in $X$. It is well known [16, Lemma 2.10, page 55] that $N(\sigma)$ collapses onto $\sigma$ and, since $\sigma$ collapses to a point, the second derived neighborhood $N(\sigma)$ is collapsible.

It is easy to see that if $\sigma$ and $\tau$ are two closed simplices of $X$ then

$$
N(\sigma) \cap N(\tau)=N(\sigma \cap \tau),
$$

with the convention that $N(\varnothing)=\varnothing$.

Let $\Sigma$ be a set of simplices of $X$ and set $N(\Sigma)=\bigcup_{\sigma \in \Sigma} N(\sigma)$. We prove by induction on the cardinality of $\Sigma$ that $\theta_{N(\Sigma)}$ is a quasi-isomorphism. If $\Sigma$ is empty, then so 
is $N(\Sigma)$ and by hypothesis $\theta_{\varnothing}$ is a quasi-isomorphism. Suppose that $\theta_{N\left(\Sigma^{\prime}\right)}$ is a quasiisomorphism when $\left|\Sigma^{\prime}\right|<k$ and let $\Sigma$ be of cardinality $k$. We write $\Sigma=\Sigma^{\prime} \cup\{\tau\}$ with $\left|\Sigma^{\prime}\right|<k$. Then

$$
N\left(\Sigma^{\prime}\right) \cap N(\tau)=\bigcup_{\sigma \in \Sigma^{\prime}} N(\sigma \cap \tau)=N\left(\Sigma^{\prime \prime}\right)
$$

for some $\Sigma^{\prime \prime}$ of cardinality $<k$. So by induction, $\theta_{N\left(\Sigma^{\prime}\right) \cap N(\tau)}$ is a quasi-isomorphism as well as $\theta_{N\left(\Sigma^{\prime}\right)}$. Also $\theta_{N(\tau)}$ is a quasi-isomorphism because $N(\tau)$ collapses to a point. Finally it is easy to see that the pair $\left\{N\left(\Sigma^{\prime}\right), N(\tau)\right\}$ is excisive. By the "MayerVietoris condition" in the hypotheses we deduce that $\theta_{N(\Sigma)}$ is quasi-isomorphism.

Taking for $\Sigma$ the set of all simplices of $X$, we have $N(\Sigma)=X$, and so $\theta_{X}$ is a quasi-isomorphism.

For a simplicial set $S_{\bullet}$, we consider the cosimplicial vector space $\operatorname{hom}\left(S_{\bullet}, \mathbb{R}\right)$ and its normalized cochain complex which we denote by $\mathrm{N}^{*}\left(S_{\bullet}\right)$ (in [7, Section 10 (d)], this is denoted by $\mathrm{C}^{*}\left(S_{\bullet}\right)$, but we have already reserved this notation for semi-algebraic cochains).

Lemma 6.12 Let $X$ be a semi-algebraic set and let $\left\{X_{1}, X_{2}\right\}$ be a pair of semialgebraic subsets of $X$.

(i) There is a short exact sequence

$$
\begin{aligned}
0 \rightarrow \mathrm{A}_{\mathrm{PA}}\left(\mathrm{S}_{\bullet}^{\mathrm{PA}}\left(X_{1}\right) \cup \mathrm{S}_{\bullet}^{\mathrm{PA}}\left(X_{2}\right)\right) \stackrel{r}{\rightarrow} \mathrm{A}_{\mathrm{PA}}\left(\mathrm{S}_{\bullet}^{\mathrm{PA}}\left(X_{1}\right)\right) & \oplus \mathrm{A}_{\mathrm{PA}}\left(\mathrm{S}_{\bullet}^{\mathrm{PA}}\left(X_{2}\right)\right) \\
& \stackrel{\Delta}{\rightarrow} \mathrm{A}_{\mathrm{PA}}\left(\mathrm{S}_{\bullet}^{\mathrm{PA}}\left(X_{1} \cap X_{2}\right)\right) \rightarrow 0 .
\end{aligned}
$$

(ii) For any simplicial set $S_{\bullet}$ there is a zigzag of natural quasi-isomorphisms of cochain complexes

$$
\mathrm{A}_{\mathrm{PA}}\left(S_{\bullet}\right) \stackrel{\simeq}{\longrightarrow} \cdots \simeq \mathrm{N}^{*}\left(S_{\bullet}\right) .
$$

(iii) If $\left\{X_{1}, X_{2}\right\}$ is semi-algebraically excisive, then there is a natural quasi-isomorphism of cochain complexes

$$
\mathrm{A}_{\mathrm{PA}}\left(\mathrm{S}_{\bullet}^{\mathrm{PA}}\left(X_{1} \cup X_{2}\right)\right) \stackrel{\simeq}{\longrightarrow} \mathrm{A}_{\mathrm{PA}}\left(\mathrm{S}_{\bullet}^{\mathrm{PA}}\left(X_{1}\right) \cup \mathrm{S}_{\bullet}^{\mathrm{PA}}\left(X_{2}\right)\right) .
$$

All of these properties remain true if $\mathrm{A}_{\mathrm{PA}}$ is replaced by $\mathrm{A}_{\mathrm{PL}}$ and/or $\mathrm{S}_{\bullet}^{\mathrm{PA}}$ by $\mathrm{S}_{\bullet}^{\text {sing }}$.

Proof (i) The map $r$ is defined by

$$
r\left(\left\{\Phi_{\sigma}\right\}_{\sigma \in \mathrm{S}_{\bullet}^{\mathrm{PA}}}\left(X_{1}\right) \cup \mathrm{S}_{\bullet}^{\mathrm{PA}}\left(X_{2}\right)\right)=\left(\left\{\Phi_{\sigma}\right\}_{\sigma \in \mathrm{S}_{\bullet}^{\mathrm{PA}}\left(X_{1}\right)},\left\{\Phi_{\sigma}\right\}_{\sigma \in \mathrm{S}_{\bullet}^{\mathrm{PA}}\left(X_{2}\right)}\right)
$$

and $\Delta$ by

$$
\Delta\left(\left\{\Phi_{\sigma}\right\}_{\sigma \in \mathrm{S}_{\bullet}^{\mathrm{PA}}\left(X_{1}\right)},\left\{\Psi_{\sigma}\right\}_{\sigma \in \mathrm{S}_{\bullet}^{\mathrm{PA}}\left(X_{2}\right)}\right)=\left\{\Phi_{\sigma}-\Psi_{\sigma}\right\}_{\sigma \in \mathrm{S}_{\bullet}^{\mathrm{PA}}\left(X_{1} \cap X_{2}\right)} .
$$


The surjectivity of $\Delta$ is a consequence of the extendability of APA and APL (Lemma 6.10 and [7, Lemma 10.7 (iii)]) and of [7, Proposition 10.4 (ii)]. The other parts of exactness are clear.

(ii) Theorem 10.9 of [7] connects $\mathrm{A}_{\mathrm{PL}}\left(S_{\bullet}\right)$ and $\mathrm{N}^{*}\left(S_{\bullet}\right)$ (denoted there by $C_{P L}\left(S_{\bullet}\right)$ ) by a zigzag of quasi-isomorphisms. Since $\mathrm{A}_{\mathrm{PA}}$ - is extendable, exactly the same proof gives a natural weak equivalence $\mathrm{A}_{\mathrm{PA}}\left(S_{\bullet}\right) \simeq \mathrm{N}^{*}\left(S_{\bullet}\right)$.

(iii) When the pair $\left\{X_{1}, X_{2}\right\}$ is excisive, the classical barycentric argument [13, Proposition 2.21] implies that there is a quasi-isomorphism

$$
\mathrm{N}^{*}\left(\mathrm{~S}_{\bullet}^{\mathrm{PA}}\left(X_{1}\right) \cup \mathrm{S}_{\bullet}^{\mathrm{PA}}\left(X_{2}\right)\right)=\mathrm{N}^{*}\left(\mathrm{~S}_{\bullet}^{\mathrm{PA}}\left(X_{1}\right)\right)+\mathrm{N}^{*}\left(\mathrm{~S}_{\bullet}^{\mathrm{PA}}\left(X_{2}\right)\right) \stackrel{\simeq}{\longrightarrow} \mathrm{N}^{*}\left(\mathrm{~S}_{\bullet}^{\mathrm{PA}}\left(X_{1} \cup X_{2}\right)\right)
$$

and similarly for $\mathrm{S}_{\bullet}^{\text {sing }}$. The map in (iii) is clear and the fact that it is a quasi-isomorphism is a consequence of (ii).

Proof of Theorem 6.1 We prove that $\alpha_{X}, \beta_{X}$, and $\gamma_{X}$ of Equation (20) are quasiisomorphisms for a compact semi-algebraic set $X$.

Since both the simplicial CDGAs $\mathrm{A}_{\mathrm{PL}} \bullet$ and $\mathrm{A}_{\mathrm{PA}} \bullet$ satisfy the Poincare lemma and are extendable (for $A_{P A}$ this is Lemma 6.3 and Lemma 6.10, and for $A_{P L}$ it is [7, Lemma 10.7]), Proposition 10.5 of [7] implies that $\beta_{X}$ is a quasi-isomorphism.

By Lemma 6.12 (ii), to prove that $\gamma_{X}$ is a quasi-isomorphism, it suffices to prove that the induced map

$$
\mathrm{N}^{*}\left(\mathrm{~S}_{\bullet}^{\operatorname{sing}}(X)\right) \longrightarrow \mathrm{N}^{*}\left(\mathrm{~S}_{\bullet}^{\mathrm{PA}}(X)\right)
$$

is a quasi-isomorphism. This natural transformation satisfies the hypotheses of Proposition 6.11. Indeed, if $X$ is collapsible then we have a semi-algebraic homotopy between the identity map on $X$ and the constant map, which induces an algebraic nullhomotopy on the cochain complexes $\mathrm{N}^{*}\left(\mathrm{~S}_{\bullet}^{\mathrm{PA}}(X)\right)$ and $\mathrm{N}^{*}\left(\mathrm{~S}_{\bullet}^{\operatorname{sing}}(X)\right)$, and the Mayer-Vietoris condition comes from an analogous argument as in the proof of Lemma 6.12 (iii). Therefore $\gamma_{X}$ is a weak equivalence for compact semi-algebraic sets.

For $X$ collapsible, by Lemma 6.3 we have that $\tilde{H}^{*}\left(\Omega_{\mathrm{PA}}^{*}(X)\right)=0$ and the same is true for the reduced homology of $\mathrm{A}_{\mathrm{PA}}\left(\mathrm{S}^{\mathrm{PA}}(X)\right)$ by Lemma 6.12 (ii). We deduce easily that when $X$ is collapsible or empty then $\alpha_{X}$ is a quasi-isomorphism. Suppose that $\left\{X_{1}, X_{2}\right\}$ is excisive. We have the short exact sequences of Lemma 6.7 and Lemma 6.12 (i) that yield long exact sequences in homology. Using Lemma 6.12 (iii), the five lemma implies that $\alpha$ satisfies the second hypothesis of Proposition 6.11. Therefore $\alpha_{X}$ is a quasi-isomorphism for all compact semi-algebraic sets $X$. 


\section{Monoidal equivalences}

One of the motivations for establishing the results in this paper is Kontsevich's proof of the formality of the little cubes operad in [18, Section 3]. In that proof, functors such as $C_{*}(-)$ and $\Omega_{\mathrm{PA}}^{*}(-)$ need to be extended from objects to operads, but this can only be done if these functors are symmetric monoidal (a basic reference for symmetric monoidal categories is Hovey [15]). In this section we thus study the monoidal properties of various functors such as $\mathrm{C}_{*}(-)$ and $\Omega_{\mathrm{PA}}^{*}(-)$.

The categories of semi-algebraic sets and of topological spaces, equipped with the cartesian product and the one-point space are symmetric monoidal categories. The forgetful functor $u$ : SemiAlg $\rightarrow$ Top is a strong symmetric monoidal functor, where "strong" means that the natural map $u(X) \times u(Y) \stackrel{\cong}{\rightrightarrows} u(X \times Y)$ is an isomorphism [8, Section 2.2.1].

The cross product constructed in Proposition 3.8,

$$
\times: \mathrm{C}_{*}(X) \otimes \mathrm{C}_{*}(Y) \rightarrow \mathrm{C}_{*}(X \times Y)
$$

makes the functor $\mathrm{C}_{*}$ of semi-algebraic chains from Definition 3.1 symmetric monoidal. The classical functor of singular chains

$$
\mathrm{S}_{*}^{\text {sing }}: \mathrm{Top} \longrightarrow \mathrm{Ch}_{+}(\mathbb{Z})
$$

defined as the normalized chain complex of the singular simplicial set $\mathrm{S}_{\bullet}^{\operatorname{sing}}(X)$ is also symmetric monoidal by the Eilenberg-Zilber map, and hence the same is true for the composite $\mathrm{S}_{*}^{\text {sing }} u$ defined on semi-algebraic sets.

Let $\mathcal{T}$ be a symmetric monoidal category. For us, a contravariant functor

$$
F: \mathcal{T} \longrightarrow \mathrm{CDGA}
$$

is symmetric monoidal if it is equipped with a natural map

$$
\kappa: F(X) \otimes F(Y) \longrightarrow F(X \times Y)
$$

satisfying the usual axioms and such that $F\left(\mathbf{1}_{\mathcal{T}}\right)=\mathbb{K}$.

Thus the functor $\Omega_{\mathrm{PA}}^{*}$ is symmetric monoidal through the Künneth quasi-isomorphism

$$
\begin{aligned}
\kappa: \Omega_{\mathrm{PA}}^{*}(X) \otimes \Omega_{\mathrm{PA}}^{*}(Y) & \stackrel{\simeq}{\longrightarrow} \Omega_{\mathrm{PA}}^{*}(X \times Y) \\
\alpha \otimes \beta & \longmapsto \operatorname{pr}_{X}^{*}(\alpha) \operatorname{pr}_{Y}^{*}(\beta),
\end{aligned}
$$

and the same is true for $\mathrm{A}_{\mathrm{PL}}$. 
A symmetric monoidal natural transformation is a natural transformation between two (covariant or contravariant) functors that commutes with all the monoidal and symmetry structure maps. It is a symmetric monoidal natural equivalence if it induces weak equivalences in an obvious sense (for example quasi-isomorphism if the target category is chain complexes or CDGA). Two symmetric monoidal functors are weakly equivalent if they are connected by a chain of symmetric monoidal natural equivalences.

Theorem 7.1 On the category of compact semi-algebraic sets, the symmetric monoidal contravariant functors $\Omega_{\mathrm{PA}}$ and $\mathrm{A}_{\mathrm{PL}}(u(-) ; \mathbb{R})$ are weakly equivalent.

Proof It is easy to see that the weak equivalences from Equation (20) before Proposition 6.11 are all symmetric monoidal.

Proposition 7.2 Symmetric monoidal functors $\mathrm{C}_{*}$ and $\mathrm{S}_{*}^{\mathrm{sing}} u$ are weakly equivalent.

Proof We have the chain complex $S_{*}^{\mathrm{PA}}(X)$ of semi-algebraic singular chains defined as the normalized chain complex associated to the simplicial set $\mathrm{S}_{\bullet}^{\mathrm{PA}}(X)$, and we have seen in the proof of Theorem 6.1 that there is a natural weak equivalence

$$
\mathrm{S}_{*}^{\mathrm{PA}}(X) \stackrel{\simeq}{\rightarrow} \mathrm{S}_{*}^{\text {sing }}(X) \text {. }
$$

It is clear that this equivalence is symmetric monoidal.

We also have a natural map

$$
\begin{aligned}
\mathrm{S}_{*}^{\mathrm{PA}}(X) & \longrightarrow \mathrm{C}_{*}(X) \\
\left(\sigma: \Delta^{k} \rightarrow X\right) & \longmapsto \sigma_{*}\left(\llbracket \Delta^{k} \rrbracket\right)
\end{aligned}
$$

which is also symmetric monoidal. It is a quasi-isomorphism by another application of Proposition 6.11.

For a real vector space $V$, denote its dual by

$$
V^{\vee}:=\operatorname{hom}(V, \mathbb{R}) .
$$

Thus $\left(\Omega_{\mathrm{PA}}(X)\right)^{\vee}$ is a chain complex. There is an evaluation chain map

$$
\text { ev: } \mathrm{C}_{*}(X) \otimes \mathbb{R} \rightarrow\left(\Omega_{\mathrm{PA}}(X)\right)^{\vee}
$$

defined by, for $\gamma \in \mathrm{C}_{k}(X)$,

$$
\operatorname{ev}(\gamma): \Omega_{\mathrm{PA}}^{k}(X) \longrightarrow \mathbb{R}, \quad \alpha \longmapsto\langle\alpha, \gamma\rangle .
$$

The proof of the following proposition is straightforward. 
Proposition 7.3 The evaluation map ev is a natural quasi-isomorphism and the following diagram commutes:

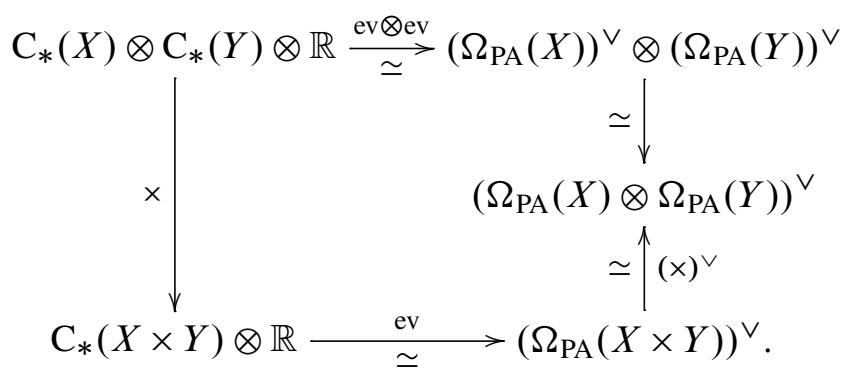

\section{Oriented semi-algebraic bundles and integration along the fiber}

A natural occurrence of a strongly continuous chain $\Phi \in \mathrm{C}_{k}^{\mathrm{str}}(p: E \rightarrow B)$ is when $p$ is a semi-algebraic bundle with fiber a compact oriented $k$-dimensional semi-algebraic manifold. The operation $f_{\Phi}$ is then a generalization of the classical integration along the fiber. In this section we define and study this notion. Many properties here will be useful in the proof of the formality of the little cubes operad [20].

First we define a locally trivial bundle in the category of semi-algebraic sets in an obvious way.

\section{Definition 8.1}

- An SA bundle is a semi-algebraic map $p: E \rightarrow B$ such that there exists a semialgebraic set $F$, a covering of $B$ by a finite family $\left\{U_{\alpha}\right\}_{\alpha \in I}$ of semi-algebraic open sets, and semi-algebraic homeomorphisms $h_{\alpha}: U_{\alpha} \times F \cong p^{-1}\left(U_{\alpha}\right)$ such that $p h_{\alpha}=\mathrm{pr}_{1}$.

- The space $F$ is called a generic fiber.

- An SA bundle is oriented if $F$ is a compact oriented manifold and each fiber $p^{-1}(b)$ is oriented in such a way that the restriction of $h_{\alpha}$ to the fiber respects the orientation.

- The fiberwise boundary of an oriented SA bundle is the oriented SA bundle

$$
p^{\partial}: E^{\partial} \longrightarrow B
$$

where

$$
E^{\partial}=\bigcup_{b \in B} \partial\left(p^{-1}(b)\right)
$$

and $p^{\partial}$ is the restriction of $p$ to $E^{\partial}$. 
Note that because we work in the category of semi-algebraic sets, asking that the covering is finite is not a restriction.

It is clear that the fiberwise boundary of an oriented SA bundle of dimension $k$ is an oriented SA bundle of dimension $k-1$.

Proposition 8.2 If $p: E \rightarrow B$ is an oriented $S A$ bundle then the formula $\Phi(b)=$ $\llbracket p^{-1}(b) \rrbracket$, for $b \in B$, defines a strongly continuous chain $\Phi \in C_{l}^{\mathrm{str}}(E \rightarrow B)$ that we call the continuous chain associated to the oriented SA bundle. Further, $\partial \Phi$ is the continuous chain associated to the fiberwise boundary $p^{\partial}: E^{\partial} \rightarrow B$.

Proof Take an open semi-algebraic cover of $X$ that trivializes the bundle and take a stratification of $B$ such that the closure of each stratum is contained in an open set of the cover. It is straightforward to construct a trivialization of $\Phi$ associated to this stratification from the trivialization of the bundle. The statement about the boundary is also straightforward.

Definition 8.3 Let $p: E \rightarrow B$ be an oriented SA bundle with fiber of dimension $k$. The pushforward or integration along the fiber operation is the degree $-k$ linear map

$$
p_{*}: \Omega_{\min }^{*+k}(E) \longrightarrow \Omega_{\mathrm{PA}}^{*}(B)
$$

defined by $p_{*}(\mu)=f_{\Phi} \mu$, where $\Phi$ is the continuous chain associated to the oriented SA bundle $p$.

This operation is very important and we now develop some of its properties.

\subsection{Properties of SA bundles}

We first have the obvious:

Proposition 8.4 The pullback of an (oriented) SA bundle along a semi-algebraic map is an (oriented) $S A$ bundle with the same fibers.

Less obvious is:

Proposition 8.5 The composite of two $S A$ bundles $p: E \rightarrow B$ and $q: B \rightarrow X$ is an $S A$ bundle. Moreover, if $p$ and $q$ are oriented then so is $q \circ p$ and

$$
\operatorname{dim}(\operatorname{fiber}(q \circ p))=\operatorname{dim}(\operatorname{fiber}(p))+\operatorname{dim}(\operatorname{fiber}(q)) .
$$


Remark 8.6 Note that it is not true in general that the composite of two bundles is a bundle. Here is a counterexample for coverings (topological bundles with discrete fibers): Let $X$ be the Hawaiian rings which is the union of circles $\Gamma_{n}$ in the plane centered at $(0,1 / n)$ with radius $1 / n$ for $n \geq 1$. For $N \geq 1$, let $q_{N}: E_{N} \rightarrow X \times\{N\}$ be a two-fold covering such that the restriction of $q_{N}$ to $\Gamma_{N}$ is the nontrivial covering and its restriction to $\Gamma_{k}$ is trivial for $k \neq N$. Set $q=\bigsqcup_{N \geq 1} q_{N}, E=\bigsqcup_{N \geq 1} E_{N}$, and let

$$
q: E \longrightarrow \bigsqcup_{N \geq 1} X \cong X \times \mathbb{N}_{0}
$$

This map is clearly a covering. The projection $p: X \times \mathbb{N}_{0} \rightarrow X$ is another covering. But the composite $p \circ q$ is not a covering because it is not trivial on any neighborhood of the origin.

To prove Proposition 8.5, we will first need two lemmas. Let $J=[0,1]^{a} \times[0,1)^{b}$ for some integers $a, b \geq 0$ and let

$$
p: E \longrightarrow B \times J
$$

be an SA bundle. Our first task will be to prove that the composite of $p$ with the projection on the second factor $J$ is an SA bundle.

\section{Lemma 8.7 Let}

$$
r: B \times J \rightarrow B \times J
$$

be the map defined by $r(b, u)=(b, 0)$. Then there exists a semi-algebraic map

$$
\widehat{r}: E \rightarrow E
$$

such that $p \circ \hat{r}=r \circ p$.

Proof Since the bundle is semi-algebraic, there exists a finite, and hence enumerable, covering of $B \times J$ which trivializes $p$. The lemma is then proved by an argument completely analogous to that of [17, Theorem 9.6 in Chapter 4, page 51].

Lemma 8.8 Let $p: E \rightarrow B \times J$ be an $S A$ bundle. Then the composite

$$
E \stackrel{p}{\longrightarrow} B \times J \stackrel{\mathrm{pr}_{2}}{\longrightarrow} J
$$

is also an SA bundle.

Proof Consider the inclusion $r_{0}: B \rightarrow B \times J$ defined by $r_{0}(b)=(b, 0)$ and the projection on the first factor $\mathrm{pr}_{1}: B \times J \rightarrow J$. Then $r_{0} \circ \mathrm{pr}_{1}$ is the map $r$ of Lemma 8.7. Let $E_{0}=p^{-1}(B \times\{0\}) \subset E$. This defines a bundle $p_{0}=p \mid E_{0}$ over $B \cong B \times\{0\}$. 
The pullback of $p_{0}$ along $\mathrm{pr}_{1}: B \times J \rightarrow B$ is the bundle $\left(p_{0} \times\right.$ id): $E_{0} \times J \rightarrow B \times J$. By Lemma 8.7 and by universal property of the pullback we get a semi-algebraic homeomorphism

$$
h: E \stackrel{\cong}{\rightrightarrows} E_{0} \times J
$$

such that $p=\left(p_{0} \times \mathrm{id}\right) \circ h$. Let $\mathrm{pr}_{2}: B \times J \rightarrow J$ be the projection on the second factor. The composite $\operatorname{pr}_{2} \circ\left(p_{0} \times \mathrm{id}\right)$ is a trivial SA bundle and, since $h$ is a homeomorphism, we deduce that $p r_{2} \circ\left(p_{0} \times \mathrm{id}\right) \circ h=\mathrm{pr}_{2} \circ p$ is also an SA bundle.

Proof of Proposition 8.5 Take a semi-algebraic covering of $X$ trivializing $q$. By [12, Theorem 2] this open covering can be refined into a semi-algebraic triangulation. Each $d$-simplex $\sigma$ is the union of exactly $d+1 d$-dimensional semi-open cubes whose vertices are the barycenters of the various faces of $\sigma$. This gives a finite covering $\left\{J_{\alpha}\right\}$ of $X$. By triviality of $q$ over $\sigma$, we have semi-algebraic homeomorphisms

$$
g_{\alpha}: J_{\alpha} \times F \stackrel{\cong}{\rightrightarrows} q^{-1}\left(J_{\alpha}\right)
$$

such that $q \circ g_{\alpha}=\mathrm{pr}_{1}$. To prove that $q \circ p$ is an SA bundle, it is enough to prove that its restriction over $J_{\alpha}$ is one. Using the homeomorphism $g_{\alpha}$ over $J_{\alpha}$, this is a consequence of Lemma 8.8.

\subsection{Properties of integration along the fiber}

We first study naturality properties of integration along the fiber.

Proposition 8.9 Consider a pullback diagram

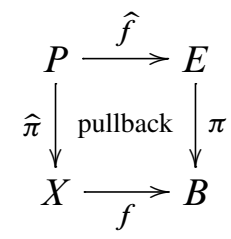

of semi-algebraic sets where $\pi$ is an oriented SA bundle. For $\mu \in \Omega_{\min }(E)$,

$$
f^{*}\left(\pi_{*}(\mu)\right)=\hat{\pi}_{*}\left(\hat{f}^{*}(\mu)\right) .
$$

Proof It is clear that if $\Phi$ is the continuous chain associated to the oriented SA bundle $\pi$, then $f^{*}(\Phi)$ is the continuous chain associated to $\hat{\pi}$ (see Definition 5.19). The formula is then straightforward.

The following is an extension of the naturality. It establishes that two SA bundles over the same base with fibers defining the same semi-algebraic chain have the same pushforward operation. This could occur for example when the two fibers are compact manifolds that differ only by some codimension 1 subspace. 
Proposition 8.10 Let $\pi: E \rightarrow B$ be an oriented bundle of dimension $k$ and $\rho: E^{\prime} \rightarrow E$ be a semi-algebraic map such that $\pi^{\prime}:=\pi \circ \rho$ is also an oriented bundle of dimension $k$, as in the diagram

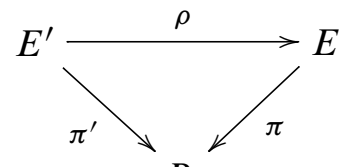

$B$.

Suppose that there is a sign $\epsilon= \pm 1$ such that for each $b \in B$,

$$
\rho_{*}\left(\llbracket \pi^{\prime-1}(b) \rrbracket\right)=\epsilon \cdot \llbracket \pi^{-1}(b) \rrbracket .
$$

Then for any minimal form $\mu \in \Omega_{\min }^{*}(E)$,

$$
\pi_{*}^{\prime}\left(\rho^{*}(\mu)\right)=\epsilon \cdot \pi_{*}(\mu)
$$

Proof We need to prove that for $\gamma \in \mathrm{C}_{l}(B)$, evaluation for two sides of Equation (22) is the same. Take a triangulation of $B$ that trivializes $\pi^{\prime}$ and that is compatible with $\gamma$. By linearity, we can restrict to a single simplex and can suppose that $\gamma=\llbracket B \rrbracket$ and $\pi^{\prime}: E^{\prime}=F^{\prime} \times B \rightarrow B$ is the projection. Then by the hypothesis,

$$
\rho: F^{\prime} \times B \longrightarrow E
$$

is a representative of the continuous chain $\Phi$ associated to the oriented SA bundle $\pi$ (up to the sign $\epsilon$ ). The proposition now follows by unravelling definitions.

We have an additivity formula:

Proposition 8.11 Let $\pi: E \rightarrow B$ be an oriented $S A$ bundle with $k$-dimensional fiber. Suppose that

$$
E=\bigcup_{\lambda \in \Lambda} E_{\lambda}
$$

where $\Lambda$ is a finite set, each $E_{\lambda}$ is a semi-algebraic subset of $E$, and the restrictions

$$
\pi_{\lambda}:=\pi \mid E_{\lambda}: E_{\lambda} \longrightarrow B
$$

are oriented $S A$ bundles with $k$-dimensional fiber, the orientation being induced by that of the fibers of $\pi$.

Suppose moreover that for $\lambda_{1}, \lambda_{2}$ distinct in $\Lambda$ and $b \in B$,

$$
\operatorname{dim}\left(\pi_{\lambda_{1}}^{-1}(b) \cap \pi_{\lambda_{2}}^{-1}(b)\right)<k .
$$

Then for any $\mu \in \Omega_{\min }(E)$,

$$
\pi_{*}(\mu)=\sum_{\lambda \in \Lambda} \pi_{\lambda *}\left(\mu \mid E_{\lambda}\right)
$$


Proof Let $\iota_{\lambda}: E_{\lambda} \hookrightarrow E$ be the inclusions and set

$$
\begin{aligned}
E^{\prime} & :=\coprod_{\lambda \in \Lambda} E_{\lambda} \\
\rho & :=\left(\iota_{\lambda}\right)_{\lambda \in \Lambda}: E^{\prime} \longrightarrow E \\
\pi^{\prime} & :=\left(\pi_{\lambda}\right)_{\lambda \in \Lambda}: E^{\prime} \longrightarrow B .
\end{aligned}
$$

We then have a diagram as in Proposition 8.10. The hypotheses imply that $\rho$ induces a degree 1 map between the fibres. Proposition 8.10 implies that

$$
\pi_{*}(\mu)=\pi_{*}^{\prime}\left(\rho^{*}(\mu)\right)=\sum_{\lambda \in \Lambda} \pi_{\lambda *}\left(\iota_{\lambda}^{*}(\mu)\right)
$$

We have also the following fiberwise Stokes' formula.

Proposition 8.12 Let $\pi: E \rightarrow B$ be an oriented $S A$ bundle with $k$-dimensional fiber and let $\pi^{\partial}: E^{\partial} \rightarrow B$ be its fiberwise boundary. For $\mu \in \Omega_{\min }(E)$,

$$
d\left(\pi_{*}(\mu)\right)=\pi_{*}(d(\mu))+(-1)^{\operatorname{deg}(\mu)-k^{2}} \pi_{*}^{\partial}\left(\mu \mid E^{\partial}\right) .
$$

Proof This is a direct consequence of Lemma 5.23.

The following is a formula for a double pushforward.

Proposition 8.13 Let $\pi: E \rightarrow B$ be an oriented $S A$ bundle. Let $N$ be an oriented compact semi-algebraic manifold, and consider the projection

$$
\mathrm{pr}_{1}: E \times N \longrightarrow E
$$

For $\mu \in \Omega_{\min }(E)$ and $v \in \Omega_{\min }(N)$,

$$
\left(\pi \circ \mathrm{pr}_{1}\right)_{*}(\mu \times v)=\pi_{*}(\mu) \cdot\langle v, \llbracket N \rrbracket\rangle .
$$

Proof By naturality it is enough to prove that the two forms we are comparing evaluate to the same thing on $\llbracket B \rrbracket$ when $B$ is an oriented compact semi-algebraic manifold. In that case,

$$
\begin{aligned}
\left\langle\left(\pi \circ \mathrm{pr}_{1}\right)_{*}(\mu \times v), \llbracket B \rrbracket\right\rangle & =\langle\mu \times v, \llbracket E \times N \rrbracket\rangle \\
& =\langle\mu, \llbracket E \rrbracket\rangle \cdot\langle v, \llbracket N \rrbracket\rangle \quad \text { by (5) in Proposition } 5.10 \\
& =\left\langle\pi_{*}(\mu), \llbracket B \rrbracket\right\rangle \cdot\langle v, \llbracket N \rrbracket\rangle .
\end{aligned}
$$

The following is useful in showing that certain integrals along the fiber vanish. 
Proposition 8.14 Let $\pi: E \rightarrow B$ be an oriented SA bundle that factors as $\pi=q \circ \rho$ with

$$
E \stackrel{\rho}{\longrightarrow} Z \stackrel{q}{\longrightarrow} B
$$

Suppose that for all $b \in B$,

$$
\operatorname{dim}\left(q^{-1}(b)\right)<\operatorname{dim}\left(\pi^{-1}(b)\right) .
$$

Then for all minimal forms $\mu \in \Omega_{\min }(Z)$, we have $\pi_{*}\left(\rho^{*}(\mu)\right)=0$.

Proof We need to prove that for $\gamma \in \mathrm{C}_{l}(B)$,

$$
\left\langle\pi_{*}\left(\rho^{*}(\mu)\right), \gamma\right\rangle=0 .
$$

Passing to a suitable triangulation of $B$, by naturality it is enough to prove this when $B$ is a compact oriented semi-algebraic manifold, $\gamma=\llbracket B \rrbracket$, and $\pi$ is trivial over $B$ with $E=F \times B$ for a compact oriented manifold $F$. >From the hypothesis we get that

$$
\operatorname{dim}(Z) \leq \operatorname{dim} B+\sup _{b \in B}\left(\operatorname{dim} q^{-1}(b)\right)<\operatorname{dim}(B \times F) .
$$

For degree reasons, $\rho^{*}(\mu)=0$ in $\Omega_{\min }(F \times B)$.

Our last proposition concerns multiplicative properties of the pushforward.

Proposition 8.15 Consider a pullback diagram

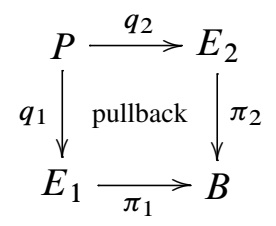

of semi-algebraic sets. Assume that $\pi_{1}$ and $\pi_{2}$ are oriented $S A$ bundles and set $\pi=\pi_{i} \circ q_{i}$. Let $\mu_{i} \in \Omega_{\min }\left(E_{i}\right)$ be a minimal form. Then $\pi$ is an oriented SA bundle and

$$
\pi_{1 *}\left(\mu_{1}\right) \wedge \pi_{2 *}\left(\mu_{2}\right)=\pi_{*}\left(q_{1}^{*}\left(\mu_{1}\right) \wedge q_{2}^{*}\left(\mu_{2}\right)\right) .
$$

Proof By Proposition 8.5, $\pi$ is an oriented SA bundle. We have a pullback

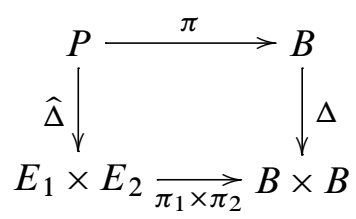

with $\widehat{\Delta}=\left(q_{1} \times q_{2}\right) \circ \Delta_{P}$, where $\Delta_{P}: P \rightarrow P \times P$ is the diagonal map. 
Using Proposition 8.9, we have

$$
\begin{aligned}
\pi_{1 *}\left(\mu_{1}\right) \wedge \pi_{2 *}\left(\mu_{2}\right) & =\Delta^{*}\left(\pi_{1 *}\left(\mu_{1}\right) \times \pi_{2 *}\left(\mu_{2}\right)\right) \\
& =\Delta^{*}\left(\left(\pi_{1} \times \pi_{2}\right) *\left(\mu_{1} \times \mu_{2}\right)\right) \\
& =\pi_{*}\left(\widehat{\Delta}^{*}\left(\mu_{1} \times \mu_{2}\right)\right) \\
& =\pi_{*}\left(\Delta_{P}^{*}\left(\left(q_{1} \times q_{2}\right)^{*}\left(\mu_{1} \times \mu_{2}\right)\right)\right. \\
& =\pi_{*}\left(q_{1}^{*}\left(\mu_{1}\right) \wedge q_{2}^{*}\left(\mu_{2}\right)\right) .
\end{aligned}
$$

An interesting example of an oriented SA bundle which is not smooth is the projection $\pi: C[n+q] \rightarrow C[n]$ between Fulton-MacPherson compactifications of configurations spaces in $\mathbb{R}^{N}$. See [20] for details.

\section{Differences between this paper and [19, Appendix A]}

The present paper is to a large extent a development of the ideas in [19, Appendix 8]. However, we have not succeeded in going as far as Kontsevich and Soibelmann suggest might be possible. We discuss this in detail in the present section.

\subsection{The scope of the equivalence $\Omega_{\mathrm{PA}}^{*} \simeq \mathrm{A}_{\mathrm{PL}}(-; \mathbb{R})$}

It is suggested in [19, Appendix 8] that the weak equivalence $\Omega_{\mathrm{PA}}^{*}(X) \simeq \mathrm{A}_{\mathrm{PL}}(X ; \mathbb{R})$ holds for all semi-algebraic sets, and even for a larger class of spaces called PA spaces (this is why our forms are called PA forms, following the notation in [19, Appendix 8]). Our proof, however, only holds for compact semi-algebraic sets. It seems reasonable though that a modification of the proof of Proposition 7.2 might produce the desired equivalence for noncompact sets as well. The problem is that we require polyhedra to be collapsible in the Poincaré lemma, but such a notion does not seem to exist for semi-open simplices.

\subsection{Strongly and weakly continuous families of chains}

The definition of a continuous family of chains in [19, Definition 22] is different from ours (Definition 5.13). The definition in that paper is probably equivalent to the following definition of weakly continuous family of chains (we say "probably" because the condition (d) in [19, Definition 22] is somewhat informal and we have interpreted it as the condition (wc) below). 
Definition 9.1 Let $f: Y \rightarrow X$ be a semi-algebraic map. A weakly continuous family of chains or, shortly, a weakly continuous chain of dimension $l$ over $X$ along $f$ is a map

$$
\Phi: X \longrightarrow \mathrm{C}_{l}(Y)
$$

such that there exist:

(1) a finite semi-algebraic stratification $\left\{S_{\alpha}\right\}_{\alpha \in I}$ of $X$; and, for each $\alpha \in I$,

(2) an oriented compact semi-algebraic manifold $F_{\alpha}$ of dimension $l$;

(3) a semi algebraic map $g_{\alpha}: S_{\alpha} \times F_{\alpha} \rightarrow Y$ satisfying:

(a) the diagram

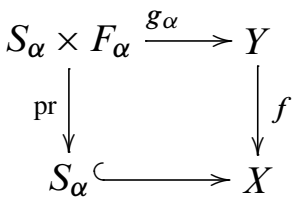

commutes;

(b) for each $\alpha \in I$ and $x \in S_{\alpha}, \Phi(x)=g_{\alpha *}\left(\llbracket\{x\} \times F_{\alpha} \rrbracket\right)$;

and such that

$$
\text { if } x_{n} \rightarrow x \text { in } X \text { then } \Phi\left(x_{n}\right) \rightarrow \Phi(x) \text { in } C_{k}(Y) \text {. }
$$

Notice that if $\Phi$ is a strongly continuous chain then it is easy to show that for any semi-algebraic curve $\theta:[0,1] \rightarrow X$ and for any sequence $\epsilon_{n}$ in $[0,1]$ converging to 0 , we have $\Phi\left(\theta\left(\epsilon_{n}\right)\right) \rightarrow \Phi(\theta(0))$. So if we had required that the sequence $\left(x_{n}\right)$ live on a semi-algebraic curve in the (wc) condition, then strongly continuous would imply weakly continuous. But with the present definition, we do not know whether such an implication is true, although this seems likely.

We now give two examples of weakly continuous chains that are not strongly continuous.

Example 9.2 Set $X=\left\{(u, v) \in \mathbb{R}^{2}: 0 \leq u \leq v \leq 1\right\}, Y=X \times[0,1]^{2}$, and let $f: Y \rightarrow X$ be the projection on the first factor. Define $\Psi: X \rightarrow \mathrm{C}_{0}(Y)$ by $\Psi(0,0)=0$ and, for $(u, v) \in X$ with $v>0$,

$$
\Psi(u, v)=\llbracket\{(u, v, u / v)\} \rrbracket \times(\llbracket\{0\} \rrbracket-\llbracket\{u v\} \rrbracket) .
$$

This parametrized family is trivialized under the stratification $\{\{(0,0)\}, X \backslash\{(0,0)\}\}$. Using the flat norm we have that $\mathbf{F}(\Psi(u, v)) \leq u v$ and this implies (wc). Therefore $\Psi$ is a weakly continuous chain. To see that it is not strongly continuous, consider the limit set of the supports of $\Psi(u, v)$ as $(u, v) \rightarrow(0,0)$, by which we mean the set

$$
\bigcap_{\epsilon>0}\left(\overline{\bigcup_{\max (|u|,|v|)<\epsilon} \operatorname{spt} \Psi(u, v)}\right) \text {. }
$$


Indeed this limit set is $\{(0,0)\} \times[0,1] \times\{0\}$ which is one-dimensional, and this is impossible in the case of a strongly continuous 0 -chain.

Example 9.3 Set $X, Y$, and $f: Y \rightarrow X$ to be the same as in the previous example. Define $\Phi: X \rightarrow \mathrm{C}_{1}(Y)$ by $\Phi(0,0)=0$ and, for $(u, v) \in X$ with $v>0$,

$$
\Phi(u, v)=\llbracket\{(u, v)\} \times[0, u / v] \rrbracket \times(\llbracket\{0\} \rrbracket-\llbracket\{u v\} \rrbracket) .
$$

The trivializing set $\{\{(0,0)\}, X \backslash\{(0,0)\}\}$, same as before. Similarly, $\mathbf{F}(\Phi(u, v)) \leq 3 u v$ which implies (wc). But $\Phi$ is not strongly continuous since its boundary is not. Indeed, $\partial(\Phi)$ is a sum of a strongly continuous chain and the chain $\Psi$ from the previous example.

As will appear later in the discussion, strongly continuous chains have certain disadvantages and the reader might wonder why we did not use weakly continuous chain instead. The problem with weakly continuous chains was the proof of the Leibniz formula

$$
\partial(\gamma \ltimes \Phi)=\partial(\gamma) \ltimes \Phi \pm \gamma \ltimes \partial(\Phi)
$$

which is needed in Lemma 5.23 (proving that $\Omega_{\mathrm{PA}}^{*}$ is a cochain subcomplex). Another problem with the condition (wc) is that, as demonstrated at the beginning of Section 4, weak convergence in even the flat semi-norms is not well adapted in the study of semi-algebraic chains. In Equation (13) of the proof of Lemma 5.29 (continuity principle for PA forms), we used strong continuity property to deduce from $\gamma_{n} \stackrel{\text { SA }}{\rightarrow} \gamma$ that $\gamma_{n} \ltimes \Phi \stackrel{\text { SA }}{\rightarrow} \gamma \ltimes \Phi$ (in the special case of a constant continuous chain $\Phi=\widehat{F}$ ). If we had worked with weakly continuous chains, we would first have had to prove from (wc) that $\gamma_{n} \ltimes \Phi \stackrel{\text { SA }}{\rightarrow} \gamma \ltimes \Phi$, which seems nontrivial. Alternatively, we would have needed to show that $\gamma_{n} \ltimes \Phi \rightarrow \gamma \ltimes \Phi$ and to deduce that $\left\langle f_{\Phi} \mu, \gamma_{n}\right\rangle \rightarrow\left\langle f_{\Phi} \mu, \gamma\right\rangle$ without SA convergence, and hence without using Proposition 5.6. This also seems quite difficult because of the example at the beginning of Section 4 .

Kontsevich suggested to us another possible definition of continuity which could perhaps be used instead of (wc). First, it should be enough to check continuity by restricting the parametrized family to any semi-algebraic curve in $X$ (although the proof of Leibniz formula could now be problematic), so we now restrict our attention to the case of semialgebraic maps $f: Y \rightarrow[0,1]$. A family of $k$-chains in $Y$ parametrized by $[0,1]$ gives a $(k+1)$-dimensional smooth oriented submanifold $M_{0} \subset Y$, with multiplicities on each connected component, such that $\left.f\right|_{M_{0}}$ is a submersion over all but a finite number of points $[0,1]$ and such that over a regular value $t \in[0,1], \Phi(t)=\llbracket M_{0} \cap f^{-1}(t) \rrbracket$. There is then a $(k+1)$-current $T=\llbracket M_{0} \rrbracket \in \mathrm{C}_{k+1}(Y)$. A continuity condition could 
then be expressed as

$$
\forall t \in[0,1]: \operatorname{dim}\left(\operatorname{spt}(T) \cap f^{-1}(t)\right) \leq k \text { and } \operatorname{dim}\left(\operatorname{spt}(\partial T) \cap f^{-1}(t)\right) \leq k-1 .
$$

\subsection{Complexity of the proof of the Poincaré lemma}

The reader might be surprised by the complexity of our proof of the Poincaré Lemma 6.3 and in particular by our need for the Hauptvermutung for semi-algebraic spaces. Let us explain why following an obvious and more natural line of proof does not work. The simplest idea would be to convert a semi-algebraic homotopy $h: X \times I \rightarrow X$, $I=[0,1]$, into a homotopy operator

$$
\Theta_{h}: \Omega_{\mathrm{PA}}^{*}(X) \longrightarrow \Omega_{\mathrm{PA}}^{*-1}(X)
$$

such that $\delta \Theta_{h} \pm \Theta_{h} \delta=(h(-, 1))^{*}-(h(-, 0))^{*}$. To try to construct such a $\Theta_{h}$, first notice that given a continuous chain $\Phi \in \mathrm{C}_{l}^{\mathrm{str}}(Y \rightarrow X)$ and any cochain $\lambda \in \mathrm{C}^{k+l}(Y)$ we can define a cochain $f_{\Phi} \lambda \in \mathrm{C}^{k}(X)$ by

$$
\left\langle f_{\Phi} \lambda, \gamma\right\rangle=\langle\lambda, \gamma \times \Phi\rangle
$$

(even if $\lambda$ is not a minimal form as in Equation (8).) For a PA form $\alpha=f_{\Phi} \mu \in \Omega_{\mathrm{PA}}^{k}(X)$ with $\mu \in \Omega_{\min }^{k+l}(X)$, we would like to define the cochain $\Theta_{h}(\alpha)$ through the equations

$$
\begin{aligned}
\left\langle\Theta_{h}\left(f_{\Phi} \mu\right), \gamma\right\rangle & =\left\langle f_{\Phi} \mu, h_{*}(\gamma \times \llbracket I \rrbracket)\right\rangle \\
& =\left\langle h^{*}\left(f_{\Phi} \mu\right), \gamma \times \llbracket I \rrbracket\right\rangle \\
& =\left\langle f_{h^{*}(\Phi)} h^{*}(\mu), \gamma \times \llbracket I \rrbracket\right\rangle \\
& =\left\langle f_{\widehat{I}}\left(f_{h^{*}(\Phi)} h^{*}(\mu)\right), \gamma\right\rangle
\end{aligned}
$$

where $\widehat{I}$ is the constant chain in $\mathrm{C}_{1}^{\mathrm{str}}(\operatorname{pr}: X \times I \rightarrow X)$.

Suppose we knew that the operation $\ltimes$ defined in Proposition 5.17 could be extended to a natural operation

$$
\ltimes: \mathrm{C}_{p}^{\mathrm{str}}(X \rightarrow T) \otimes \mathrm{C}_{l}^{\mathrm{str}}(Y \rightarrow X) \rightarrow \mathrm{C}_{p+l}^{\mathrm{str}}(Y \rightarrow T)
$$

by the formula

$$
(\Gamma \ltimes \Phi)(t)=(\Gamma(t)) \ltimes \Phi,
$$


where $\Gamma \in \mathrm{C}_{p}^{\mathrm{str}}(X \rightarrow T), \Phi \in \mathrm{C}_{l}^{\mathrm{str}}(Y \rightarrow X)$, and $t \in T$. In this case we could set

$$
\Theta_{h}\left(f_{\Phi} \mu\right):=f_{\widehat{I} \ltimes h^{*} \Phi} h^{*}(\mu) \in \Omega_{\mathrm{PA}}^{k-1}(X)
$$

and Equations (23) would show that $\Theta_{h}$ is an algebraic homotopy between $\left.h(-, 0)\right)^{*}$ and $(h(-, 1))^{*}$. This would of course imply the Poincaré lemma.

The problem is that the twisted product $\Gamma \ltimes \Phi$ is in general not a strongly continuous chain, as we can see in the following.

Example 9.4 Let $X$ be the convex hull of $\{(0,0,0),(1,0,0),(1,1,0),(0,0,2)\}$ in $\mathbb{R}^{3}$ (a closed 3-simplex) and let $t_{1}, t_{2}, t_{3}$ be coordinates in $\mathbb{R}^{3}$. Consider the surface

$$
S=\left\{\left(t_{1}, t_{2}, t_{3}\right) \in X: t_{2}=t_{1} t_{3}\right\}
$$

and the subspace below it and lying in $X$,

$$
E=\left\{\left(t_{1}, t_{2}, t_{3}\right) \in X: t_{2} \geq t_{1} t_{3}\right\} .
$$

Set $Y=E \times I$ and $f: Y \stackrel{\mathrm{pr}}{\rightarrow} E \hookrightarrow X$. For $x \in X$ consider the distance function to $S, d: X \rightarrow I$, defined by $d(x)=\operatorname{dist}(x, S)$. Define a continuous 0-chain $\Phi \in \mathrm{C}_{0}^{\mathrm{str}}(Y \rightarrow X)$ by $\Phi(x)=\llbracket\{x\} \rrbracket \times(\llbracket\{0\} \rrbracket-\llbracket\{d(x)\} \rrbracket)$ if $x \in E$ and $\Phi(x)=0$ otherwise. This is a strongly continuous chain trivialized by the stratification $\{E, X \backslash E\}$.

Define $H: X \times I \rightarrow X$ such that $H(-, t)$ is a dilation of center $(0,0,2)$ and coefficient $t \in[0,1]$. Consider the pullback

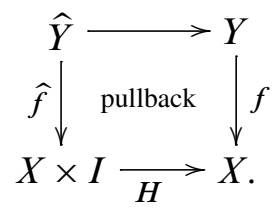

So we have the continuous chains $H^{*}(\Phi) \in \mathrm{C}_{0}^{\mathrm{str}}(\widehat{Y} \rightarrow X \times I)$ and $\hat{I} \in \mathrm{C}_{1}^{\mathrm{str}}\left(\operatorname{pr}_{1}: X \times I \rightarrow X\right)$.

However, $\widehat{I} \ltimes H^{*}(\Phi)$ does not define a strongly continuous chain. To see this, first notice that the preimage of $\left(t_{1}, t_{2}, 0\right) \in X$ with $0<t_{2} \leq t_{1} \leq 1$ under $H \circ \hat{f}$ is homeomorphic to

$$
\left\{\left(t_{1}, t_{2}, 0, t_{4}, t_{5}\right) \mid 1-\frac{t_{2}}{2 t_{1}} \leq t_{4} \leq 1 \text { and } 0 \leq t_{5} \leq 1\right\},
$$


where $t_{4}$ is the last coordinate of $X \times I$, and $t_{5}$ is the last coordinate of $Y=E \times I$. The chain $\widehat{I} \ltimes H^{*}(\Phi)$ at $\left(t_{1}, t_{2}, 0\right)$ is a difference $I_{0}-I_{1}$ where $I_{0}$ and $I_{1}$ are given by

$$
\begin{aligned}
I_{0}, I_{1}:\left[1-\frac{t_{2}}{2 t_{1}}, 1\right] & \longrightarrow(H \circ \widehat{f})^{-1}\left(t_{1}, t_{2}, 0\right) \\
I_{0}: t_{4} & \longmapsto\left(t_{1}, t_{2}, 0, t_{4}, 0\right) \\
I_{1}: t_{4} & \longmapsto\left(t_{1}, t_{2}, 0, t_{4}, d\left(t_{1} t_{4}, t_{2} t_{4}, 2-2 t_{4}\right)\right)
\end{aligned}
$$

For a fixed $\lambda \in(0,1]$ it is easy to see that $\operatorname{spt}\left(\left(\widehat{I} \ltimes H^{*}(\Phi)\right)\left(t_{1}, \lambda \cdot t_{1}, 0\right)\right)$ tends to the limit set $\{(0,0,0)\} \times[1-\lambda / 2,1] \times\{0\}$ as $t_{1} \rightarrow 0_{+}$.

Now assume that $\widehat{I} \ltimes H^{*}(\Phi)$ is strongly trivialized by $\left\{S_{\alpha}, F_{\alpha}, g_{\alpha}\right\}_{\alpha \in J}$. We will say that a curve in $X$ passing through $(0,0,0)$ is locally in $S_{\alpha}$ if $\overline{S_{\alpha}}$ contains a neighborhood of $(0,0,0)$ in that curve. Obviously any such curve should be locally in at least one $S_{\alpha}$. Let us show that, for any $0<a<b<1$, the curves $\{(t, a t, 0) \mid 0 \leq t \leq 1\}$ and $\{(t, b t, 0) \mid 0 \leq t \leq 1\}$ cannot be locally in the same $S_{\alpha}$. This would then give a necessary contradiction (since there are infinitely many such curves, two of them must be locally in the same stratum). Applying (25) to the points from the first curve and taking the limit set as $t \rightarrow 0$, we get

$$
g_{\alpha}\left(\{(0,0,0)\} \times F_{\alpha}\right) \subset\{(0,0,0)\} \times[1-a / 2,1] \times[0,1] .
$$

But applying (26) to the points from the second curve and again taking the limit set as $t \rightarrow 0$ we get

$$
g_{\alpha}\left(\{(0,0,0)\} \times F_{\alpha}\right) \supset\{(0,0,0)\} \times[1-b / 2,1] \times\{0\} .
$$

Since $a<b$ these two conditions can not be satisfied simultaneously.

We do not know whether the operation $\ltimes$ can be extended as in equation (24) using weakly continuous chains.

\subsection{Integration along the fiber of a PA form}

At the end of [19, Appendix 8], Kontsevich and Soibelman claim that, given an oriented SA bundle $p: E \rightarrow B$ with fiber of dimension $l$, there exists a pushforward operation

$$
p_{*}: \Omega_{\mathrm{PA}}^{*}(E) \longrightarrow \Omega_{\mathrm{PA}}^{*-l}(B)
$$

given by integration along the fiber. It is certainly true that there is a map

$$
\begin{aligned}
p_{*}: \Omega_{\min }^{*}(E) & \longrightarrow \Omega_{\mathrm{PA}}^{*-l}(B) \\
\mu & \longmapsto f_{\Phi} \mu
\end{aligned}
$$


defined on minimal forms, where $\Phi \in \mathrm{C}_{l}^{\mathrm{str}}(E \rightarrow B)$ is the continuous chain associated to this oriented SA bundle, as we have seen in Section 8. However, we were unable to prove that there is an extension to all PA forms. A natural candidate for the pushforward $\int_{\Psi} \mu \in \Omega_{\mathrm{PA}}^{k}(X)$, where $\Psi \in \mathrm{C}_{p}^{\mathrm{str}}(Y \rightarrow E)$ and $\mu$ a PA form in $\Omega_{\min }^{k+p}(Y)$, is

$$
p_{*}\left(\int_{\Psi} \mu\right):=\int_{\Phi} \int_{\Psi} \mu=\int_{\Phi \ltimes \Psi} \mu,
$$

but the problem as in the previous section is that it is unclear whether $\Phi \ltimes \Psi$ is a continuous chain and therefore whether $\int_{\Phi} \int_{\Psi} \mu$ is a PA form.

One could try to avoid this problem by inductively defining $\Omega_{\mathrm{PA}}^{*}(X)$ by

$$
\begin{aligned}
\Omega_{\mathrm{PA}_{(0)}^{*}}^{*}(X) & :=\Omega_{\min }^{*}(X) \\
\Omega_{\mathrm{PA}_{(p+1)}^{*}}^{*}(X) & :=\left\{f_{\Psi} \lambda \mid \Psi \in \mathrm{C}_{*}^{\mathrm{str}}(Y \rightarrow X), \lambda \in \Omega_{\left.\mathrm{PA}_{(p)}^{*}(Y)\right\}}^{*}\right. \\
\Omega_{\mathrm{PA}_{(\infty)}^{*}}^{*}(X) & :=\bigcup_{p=0}^{\infty} \Omega_{\mathrm{PA}_{(p)}^{*}(X) .}
\end{aligned}
$$

In particular, $\Omega_{\mathrm{PA}}^{*}(X)=\Omega_{\mathrm{PA}_{(1)}^{*}}^{*}$. It is clear that one gets a well-defined pushforward operation on $\Omega_{\mathrm{PA}_{(\infty)}^{*}}^{*}$ this way and it can be proved exactly as for $\Omega_{\mathrm{PA}}$ that $\Omega_{\mathrm{PA}_{(\infty)}^{*}}^{*}$ is a cochain complex and satisfies the Poincare lemma and the other properties which makes it weakly equivalent to the cochain complex $A_{P L}(X ; \mathbb{R})$. The problem is to check that $\Omega_{\mathrm{PA}_{(\infty)}^{*}}^{*}$ is a commutative algebra. For this we would need a generalization of Proposition 5.25, whose proof was based on the integral representation of

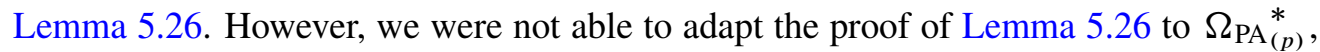
even though it seems likely that elements of $\Omega_{\mathrm{PA}_{(p)}^{*}}^{*}(X)$ are smooth on the complement of a codimension 1 subset.

\subsection{Flat morphisms}

Also at the end of [19], it is suggested that the pushforward operation exist for more general maps than bundles. More precisely, given a proper semi-algebraic map $f: Y \rightarrow X$ between oriented semi-algebraic manifolds such that, for each $x \in X$,

$$
\operatorname{dim}\left(f^{-1}\{x\}\right) \leq k-j, \quad \text { where } j=\operatorname{dim} X \text { and } k=\operatorname{dim} Y,
$$

one could construct a pushforward along $f$ as follows. The notion of slicing [6, 4.3; $9,4.3 ; 11,4.5]$ then gives a continuous chain $\Phi \in \mathrm{C}_{k-j}^{\mathrm{str}}(f: Y \rightarrow X)$ such that, for each point $x$ in the open dense set of regular values, we have $\Phi(x)=\llbracket f^{-1}\{x\} \rrbracket$, ie $\Phi(x)$ is the smooth fiber with the induced orientation. To see this, let $\llbracket Y \rrbracket$ denote the $k$ dimensional semi-algebraic chain given by oriented integration over $Y$. Then 
the correspondingly oriented graph $g_{\#} \llbracket Y \rrbracket$, where $g(y)=(y, f(y))$, is also a $k$ dimensional semi-algebraic chain. Using the projections $p(x, y)=y$ and $q(x, y)=x$ for $(y, x) \in Y \times X$, we have from $[9,4.3 ; 11,4.5]$ that the slice

$$
\langle\llbracket Y \rrbracket, f, x\rangle=p_{\#}\left\langle g_{\#} \llbracket Y \rrbracket, q, x\right\rangle
$$

is a $(k-j)$-dimensional semi-algebraic chain. The map $x \mapsto\langle\llbracket Y \rrbracket, f, x\rangle$ is also seen to be flat continuous, and hence weakly continuous (here we are implicitly using the formula $[6,4.3 .2(6)]$ which shows that the slice is invariant under an oriented diffeomorphism of the range and is thus well-defined as a map into the oriented manifold $X$ ).

While the slice $\langle\llbracket Y \rrbracket, f, x\rangle$ equals the naturally oriented fiber $\llbracket f^{-1}\{x\} \rrbracket$ for any regular value $x$ (by, for example, [6, 4.3.8 (2)]), one only knows in general that $\operatorname{spt}\langle\llbracket Y \rrbracket, f, x\rangle \subset$ $f^{-1}\{x\}$. For example, if $Y$ is the counter-clockwise oriented unit circle, $X=\mathbb{R}$, and $f(x, y)=x$, then

$$
\langle\llbracket Y \rrbracket, f, x\rangle= \begin{cases}\llbracket\left(x, \sqrt{1-x^{2}}\right) \rrbracket-\llbracket\left(x,-\sqrt{1-x^{2}}\right) \rrbracket & -1<x<1, \\ 0 & \text { otherwise, }\end{cases}
$$

which is consistent with the weak continuity of the slice even at $x= \pm 1$. Another elementary example is the formula

$$
\langle\llbracket \mathbb{C} \rrbracket, h, 0\rangle=\sum_{z \in h^{-1}\{0\}} n(h, z) \llbracket z \rrbracket,
$$

valid for any holomorphic $h: \mathbb{C} \rightarrow \mathbb{C}$, where $n(h, z)$ is the multiplicity as a zero of $h(z)$.

Applying the trivialization result of [12] to the semi-algebraic map $f$, one sees that $\Phi(x)=\langle\llbracket Y \rrbracket, f, x\rangle$ actually defines a strongly continuous chain $\Phi \in \mathrm{C}_{k-j}^{\mathrm{str}}(f: Y \rightarrow X)$.

Moreover, for any semi-algebraic chain $T \in \mathrm{C}_{\ell}(X)$ we have a pullback chain $f^{\#}(T) \in$ $\mathrm{C}_{\ell+k-j}(Y)$. This may be defined by noting that $T$ decomposes into a finite sum of chains of the form $T_{i}=\left\langle\llbracket X_{i} \rrbracket, f_{i}, 0\right\rangle$ for some open semi-algebraic subsets $X_{i}$ of $X$ and semi-algebraic maps $f_{i}: X_{i} \rightarrow \mathbb{R}^{j-\ell}$ with $\operatorname{dim}\left(X_{i} \cap f_{i}^{-1}\{0\}\right) \leq \ell$ and $\operatorname{dim}\left(\operatorname{fr}\left(X_{i}\right) \cap f_{i}^{-1}\{0\}\right) \leq \ell-1$. Then the pullback

$$
f^{\#} T=\sum_{i}\left\langle\llbracket Y \cap f^{-1}\left(X_{i}\right) \rrbracket, f_{i} \circ f, 0\right\rangle \in \mathrm{C}_{\ell+k-j}(Y)
$$

is well-defined, independent of the decomposition. Details and applications of this construction will be discussed in a later paper. 


\section{References}

[1] J Bochnak, M Coste, M-F Roy, Géométrie algébrique réelle, Ergebnisse der Math. und ihrer Grenzgebiete (3) 12, Springer, Berlin (1987) MR949442

[2] A K Bousfield, V K A M Gugenheim, On PL de Rham theory and rational homotopy type, Mem. Amer. Math. Soc. 8, no. 179 (1976) ix+94 MR0425956

[3] G Comte, J-M Lion, J-P Rolin, Nature log-analytique du volume des sous-analytiques, Illinois J. Math. 44 (2000) 884-888 MR1804313

[4] P Deligne, P Griffiths, J Morgan, D Sullivan, Real homotopy theory of Kähler manifolds, Invent. Math. 29 (1975) 245-274 MR0382702

[5] L van den Dries, Limit sets in o-minimal structures, from: "O-minimal structures, Proceedings of the RAAG Summer School (Lisbon 2003)", (M Edmundo, D Richardson, A J Wilkie, editors), Lecture Notes in Real and Algebraic Geometry (2005) 159-215

[6] H Federer, Geometric measure theory, Die Grund. der math. Wissenschaften 153, Springer, New York (1969) MR0257325

[7] Y Félix, S Halperin, J-C Thomas, Rational homotopy theory, Graduate Texts in Math. 205, Springer, New York (2001) MR1802847

[8] F Guillén Santos, V Navarro, P Pascual, A Roig, Monoidal functors, acyclic models and chain operads, Canad. J. Math. 60 (2008) 348-378 MR2398753

[9] R M Hardt, Slicing and intersection theory for chains associated with real analytic varieties, Acta Math. 129 (1972) 75-136 MR0315561

[10] R M Hardt, Stratification of real analytic mappings and images, Invent. Math. 28 (1975) 193-208 MR0372237

[11] R M Hardt, Topological properties of subanalytic sets, Trans. Amer. Math. Soc. 211 (1975) 57-70 MR0379882

[12] R M Hardt, Semi-algebraic local-triviality in semi-algebraic mappings, Amer. J. Math. 102 (1980) 291-302 MR564475

[13] A Hatcher, Algebraic topology, Cambridge Univ. Press (2002) MR1867354

[14] H Hironaka, Triangulations of algebraic sets, from: "Algebraic geometry (Proc. Sympos. Pure Math., Vol. 29, Humboldt State Univ., Arcata, Calif., 1974)”, (R Hartshorne, editor), Amer. Math. Soc. (1975) 165-185 MR0374131

[15] M Hovey, Model categories, Math. Surveys and Monogr. 63, Amer. Math. Soc. (1999) MR1650134

[16] J F P Hudson, Piecewise linear topology, Univ. of Chicago Lecture Notes, W A Benjamin, New York-Amsterdam (1969) MR0248844 Prepared with the assistance of $\mathrm{J} \mathrm{L}$ Shaneson and J Lees

[17] D Husemoller, Fibre bundles, third edition, Graduate Texts in Math. 20, Springer, New York (1994) MR1249482 
[18] M Kontsevich, Operads and motives in deformation quantization, Lett. Math. Phys. 48 (1999) 35-72 MR1718044 Moshé Flato (1937-1998)

[19] M Kontsevich, Y Soibelman, Deformations of algebras over operads and the Deligne conjecture, from: "Conférence Moshé Flato 1999, Vol. I (Dijon)", (G Dito, D Sternheimer, editors), Math. Phys. Stud. 21, Kluwer Acad. Publ., Dordrecht (2000) 255-307 MR1805894

[20] P Lambrechts, I Volić, Formality of the little $N$-disks operad, submitted arXiv: 0808.0457

[21] P Lelong, Intégration sur un ensemble analytique complexe, Bull. Soc. Math. France 85 (1957) 239-262 MR0095967

[22] C P Rourke, B J Sanderson, Introduction to piecewise-linear topology, Ergebnisse der Math. und ihrer Grenzgebiete 69, Springer, New York (1972) MR0350744

[23] M Shiota, M Yokoi, Triangulations of subanalytic sets and locally subanalytic manifolds, Trans. Amer. Math. Soc. 286 (1984) 727-750 MR760983

[24] D Sullivan, Infinitesimal computations in topology, Inst. Hautes Études Sci. Publ. Math. (1977) 269-331 (1978) MR0646078

[25] E C Zeeman, On the dunce hat, Topology 2 (1964) 341-358 MR0156351

Department of Mathematics, Rice University

6100 S Main Street, MS 136, Houston TX 77005, USA

Institut de Recherche en Mathématique et Physique

Chemin du cyclotron 2, B-1348 Louvain-la-Neuve, Belgium

Department of Mathematics, Kansas State University

Manhattan, KS 66506, USA

Mathematics Department, Wellesley College

106 Central St, Wellesley MA 02481, USA

hardt@rice.edu, pascal.lambrechts@uclouvain.be, turchin@ksu.edu, ivolic@wellesley . edu

http://math.rice.edu/ hardt, http://milnor.math.ucl.ac.be/plwiki, http://www.math.ksu.edu/ turchin, http://palmer.wellesley.edu/ ivolic

Received: 27 January 2011 